\title{
AGGRESSION IN PSYCHIATRY
} PREVENTION, EPIDEMIOLOGY \& TREATMENT

\author{
UMC Utrecht Brain Center
}

\author{
MARGO D.M. FAAY
}


Cover \& Lay-out Margo Faay \& Kristiaan Asscheman ISBN 978-90-393-7317-0

(C) 2020 Margo Faay All rights reserved. No part of this thesis may be reproduced, stored or transmitted in any form or by any means, without prior permission of the author, or, when applicable, of the publishers of the scientific papers. 


\title{
Aggression in psychiatry prevention, epidemiology \& treatment
}

\author{
Agressie in de psychiatrie \\ preventie, epidemiologie \& behandeling \\ (met een samenvatting in het Nederlands)
}

\section{Proefschrift}

ter verkrijging van de graad van doctor aan de

Universiteit Utrecht

op gezag van de

rector magnificus, prof.dr. H.R.B.M. Kummeling, ingevolge het besluit van het college voor promoties

in het openbaar te verdedigen op

woensdag 23 september 2020 des ochtends te 11.00 uur

door

Margo Désirée Maria Faay

geboren op 12 september 1987

te Waddinxveen 
Promotoren:

Prof. dr. J.J. van Os

Prof. dr. F.E. Scheepers 




\section{CONTENTS}

Chapter 1 General introduction

9

Chapter 2 Warning signs prior to aggressive behaviour in child 25 psychiatric units

Chapter 3 Kennedy Axis V: clinimetric properties assessed by 39 mental health nurses

Chapter 4 Aggressive behaviour, hostility, and associated care 59 needs in patients with psychotic disorders: a 6-year follow-up study

Chapter 5 Hostility and aggressive behaviour in first episode 79 psychosis: results from the OPTiMiSE trial

Chapter 6 Efficacy of typical and atypical antipsychotic medication on hostility in patients with psychosis-spectrum disorders: a review and meta-analysis

Chapter 7 Summary

Chapter 8 General discussion

References

Samenvatting

Dankwoord

Curriculum Vitae 



\section{CHAPTER 1}

General Introduction 



\section{General introduction}

Aggression in psychiatric practice is the focus of this thesis. Aggression can be a major disruption in the process of care and causes significant risks to everyone involved. Years of research has led to a number of clinically relevant findings, many of which are currently used in psychiatric practice. However, aggression is difficult to treat and to study and remains a major obstacle in clinical practice.

The overarching aim of this thesis is to contribute to current knowledge about aggression in psychiatry with a focus on clinically relevant results and insights. In the following chapters, three central themes are addressed: prevention, epidemiology and treatment. The chapters all have a different aim and focus that are explored in four different psychiatric populations: child inpatients (Chapter 2), adult and adolescent in- and outpatients (Chapter 3), patients with First Episode Psychosis (FEP; Chapter 5) and patients with psychotic disorders (Chapters $4 \& 6$ ). As aggression is a problem that affects all health care workers, the focus is multidisciplinary and not only on nursing interventions or clinician-related topics. This first chapter introduces the phenomenon of aggression in psychiatry and explains how previous research supports current clinical practice.

\section{The problem of aggression in psychiatry}

Aggression in psychiatry has numerous consequences for mental health care workers and patients. Almost all mental health care workers experience aggression at one point during their career. One study found that nurses have a chance of 1 in 10 each year of receiving some kind of injury as a result from patient aggression (Foster, Bowers, \& Nijman, 2007). Workplace aggression is associated with burn-out in nurses (Lopez-Lopez et al., 2019; Yang, Stone, Petrini, \& Morris, 2018) and with a reduced professional quality of life (Itzhaki et al., 2018).

Consequences for patients include prolonged hospitalization, legal consequences and physical injuries (Taylor, 2008). Aggression can lead to coercive interventions such as seclusion, restraint and medication. These coercive interventions have physical and psychological consequences and can lead to post-traumatic stress disorder (Chieze, Hurst, Kaiser, \& Sentissi, 2019). Furthermore, aggression has social consequences such as individual stigmatization and patients with hostile attributions suffer from poorer social functioning (Lahera et al., 2015).

Stigmatization in general is an important consequence of aggressive behaviour in patients with mental disorders. There is a certain public fear about "violent psychiatric patients." Many people believe that patients with mental illness are unpredictable and 
violent. This contributes to the stigmatization of patients (Arboleda-Florez, 2003). The media contributes to this with sensational reports on aggression by people with mental illness (Varshney, Mahapatra, Krishnan, Gupta, \& Deb, 2016).

The majority of aggressive incidents in society are not caused by patients with mental disorders. Only a small proportion of patients with mental disorders are at risk for displaying aggressive behaviour (Ose, Lilleeng, Pettersen, Ruud, \& van Weeghel, 2017). Patients with mental disorders have a higher risk of becoming the victim of aggression than being the perpetrator (Desmarais et al., 2014; Teplin, McClelland, Abram, \& Weiner, 2005). Therefore, the public belief that all patients with mental disorders are dangerous and violent appears to be based on unfunded assumptions.

Studying aggression in psychiatry could contribute to further stigmatization as this confirms that aggression is a problem. However, it has been argued that the most effective way of decreasing stigma is treatment of these kinds of behaviours (Torrey, 2011). Research towards understanding and preventing aggression in psychiatry could contribute to a decrease in incidents. This does appear to form at least the beginning of a justification for the need for research.

\section{Definitions and subtypes of aggression}

Hostility, aggression and violence are used to describe the same kind of behaviour. However, there are differences between these concepts.

\section{The definitions of hostility, aggression and violence}

Hostility is defined as "the state or fact of being hostile; hostile action exercised by one community, state, or power against another" but also as "opposition or antagonism in action, thought, or principle" (Oxford English Dictionary, 2019b). Synonyms include: "unfriendliness," "hatred" and "aggression" (Oxford Dictionary \& Thesaurus, 2010b).

Aggression is defined as "the practice of attacking another or others; the making of an attack or assault" or "behaviour intended to injure another person or animal" (Oxford English Dictionary, 2019a). Synonyms of aggression include "hostile acts or behaviours" (Oxford Dictionary \& Thesaurus, 2010a). A more classic definition is that of Buss from 1961: "a response that delivers noxious stimuli to another organism" (Buss, 1961). While this definition does not include auto-aggression, auto-aggression is included in other definitions. A common definition of aggression used in mental health research is "any verbal, non-verbal, or physical behaviour that was threatening (to self, others or property), or physical behaviour that actually did harm to self, others, or property" (Morrison, 1990). 
Violence is defined as "the deliberate exercise of physical force against a person, property, etc." (Oxford English Dictionary, 2019c). The World Health Organization (WHO) defines violence as "the intentional use of physical force or power, threatened or actual, against oneself, another person, or against a group or community that either results in or has a high likelihood of resulting in injury, death, psychological harm, maldevelopment or deprivation" (World Health Organization, 1996).

With these definitions, the first problem with studying aggression is also identified. There is not a single definition that perfectly covers these concepts and aggression is still poorly defined (Connor et al., 2019; Lee, 2015;Volavka, 2008). The lack of a universal definition complicates not only research but also practice.

In research, "violence" is traditionally used in criminology, sociology, law, and public policy while "aggression" is used in biomedical or psychological research (Volavka, 2008). In clinical practice, "hostility" is used to indicate symptoms such as anger and unfriendliness. "Aggression" is used to describe a variety of acts, verbal and physical. "Violence" is seen as more serious and usually only indicates physical violence.

\section{Definitions used in this thesis}

This thesis includes all aggressive behaviour towards others: physical and verbal, severe and less severe. Therefore, the term "aggression" is used. Aggression towards self is not included in this thesis.

Chapters 4, 5 and 6 focus on aggression in patients with psychotic disorders. Many previous studies towards psychotic disorders, specifically pharmacological research, unfortunately do not include a separate measure of aggression. However, commonly used instruments for the assessment of psychiatric symptoms, such as the Positive and Negative Syndrome Scale (PANSS (Kay, Fiszbein, \& Opler, 1987)) and the Brief Psychiatric Rating Scale (BPRS (Overall \& Gorham, 1962)), include a hostility item. Therefore, hostility is often the best available indicator for aggressive behaviour and is sometimes used as a proxy, including in some chapters of this thesis. The PANSS definition of hostility is "verbal and nonverbal expressions of anger and resentment, including sarcasm, passive aggressive behaviour, verbal abuse and assaultiveness" (Kay et al., 1987). The BPRS hostility item is defined as: "animosity, contempt, belligerence, disdain for other people" (Overall \& Gorham, 1962). In this thesis, hostility indicates behaviours as defined in the PANSS or BPRS.

\section{Subtypes of aggression}

Aggression is a heterogeneous concept that includes a range of disruptive behaviours, from verbal aggression to severe physical incidents. The lack of a universal definition 
urges the need for some kind of classification. It has been discussed that a better understanding of the different types of aggression helps clinicians to examine these behaviours and facilitate more targeted interventions (Connor et al., 2019).

The difference between verbal and physical aggression is well-known and frequently used in clinical practice and in research. In children, where aggression can be age-normative, a distinction can be made between adaptive and maladaptive aggression. Adaptive aggression is seen as "appropriate," for example to ensure one's safety, while maladaptive aggression is "excessive or inappropriate" and should be treated (Connor, 2012). An interesting categorization derives from the approach of aggression as a medical syndrome. Aggression can be deconstructed into psychotic, organized and impulsive aggression, each with a hypothetically distinct neurobiological basis (Stahl, 2014). This categorization gives an indication for different treatment options. Psychotic aggression can be derived from delusions or command hallucinations. While psychotic aggression can be dangerous because of its unpredictability, this does implicate clear treatment options - reducing the hallucinations or delusions would reduce the aggression risk. Discriminating impulsive and organized aggression appears to be in line with previously described subtypes of aggression: proactive and reactive aggression.

The dichotomization between proactive (or instrumental, organized or premeditated) aggression and reactive (or impulsive, hostile or affective) aggression has a long tradition in research (Cornell et al., 1996; Crick \& Dodge, 1996; Kockler, Stanford, Nelson, Meloy, \& Sanford, 2006). Proactive aggression is defined as "harmful behaviour engaged in without provocation to obtain an outcome or coerce others" (Del Vecchio, 2011). Proactive aggression is seen as deliberate or planned and could be linked to empathy dysfunctions (Blair, 2016). Reactive aggression is defined as "committed in response to a perceived threat of insult" (Brosbe, 2011). Reactive aggression is not planned but mostly based on impulses, emotions or symptoms and can derive from agitation, frustration or irritation.

There are suggestions that the dichotomization between proactive and reactive aggression should be ended as it fails to consider multiple motives, while aggression often contains both anger and a planned component (Bushman \& Anderson, 2001). A meta-analysis found a high correlation between proactive and reactive aggression in children and adolescents (Card \& Little, 2006). Other findings suggest that the distinction between proactive and reactive aggression is mainly driven by the severity of aggression, both in adults (Brugman et al., 2017) and in adolescents (Smeets et al., 2017). 


\section{The prevalence of aggression in psychiatry}

The problem with the operationalisation of aggressive behaviour extends to its measurement. The incidence and prevalence of aggression in different psychiatric populations has been studied. There are numerous studies, reviews and meta-analysis, but these reports differ in definition and severity of aggression (ranging from verbal aggression to severe incidents), data collection (self-report or observational data) and population (in- or outpatients, with or without previous aggression). Methodological issues include the use of retrospective or cross-sectional data, differences in time frames, and selection bias, as patients with aggressive behaviours are less likely to participate in trials. The following section describes some results on the prevalence of aggression within the populations discussed in this thesis: inpatient settings, patients with psychotic disorders, patients with FEP and child psychiatric facilities.

\section{Inpatient settings}

A review including data on inpatient aggression from 122 studies, found that the mean prevalence was $32.4 \%$, categorizing patients as aggressive and non-aggressive (Bowers, Stewart, Papadopoulos, Dack, \& Ross, 2011). However, as this is a report on 122 studies, heterogeneity is high. Next to differences in definitions and measures of aggression, it is unclear if these studies measure prevalence at a single point in time (point prevalence) or during a specific period (period prevalence), for example over the past week or during admission. Further, expressed in occupied bed days, this review found a mean of $3.14(S D=5.4)$ aggressive incidents per 100 bed days (Bowers et al., 2011). A meta-analysis, also aimed at inpatient aggression, included 35 studies with 23,972 patients on acute psychiatric wards and found that $17 \%$ (95\% CI=14$20 \%$ ) committed at least one act of aggression during admission (Iozzino, Ferrari, Large, Nielssen, \& de Girolamo, 2015).

\section{Patients with psychotic disorders}

A meta-analysis of 20 studies including 18,423 patients with psychotic disorders, found the point prevalence of aggression at $9.9 \%$ (Fazel, Gulati, Linsell, Geddes, \& Grann, 2009). The Odds Ratio (OR) for the risk of aggressive behaviour in patients with schizophrenia was 5.5 (95\% $C I=4.1-7.5)$, but heterogeneity was high $\left(I^{2}=96\right)$ (Fazel et al., 2009). A more recent meta-analysis, including 45,533 in- and outpatients with psychotic disorders from 110 studies, found a point prevalence of $18.5 \%$ (Witt, van Dorn, \& Fazel, 2013). 


\section{Patients with first episode psychosis}

Patients with FEP are specifically at risk for aggressive behaviour. A clinical cohort study followed 109 patients for 12 months after first service contact and found that $25.7 \%$ perpetrated any physical violence and $9.2 \%$ was arrested or charged for violent offences (Whiting, Lennox, \& Fazel, 2019).

A 10-year prospective follow-up study found that $20 \%$ of the 178 FEP patients was arrested or incarcerated at least once during these 10 years (Langeveld et al., 2014). A meta-analysis, including 6 studies, found that the pooled estimate proportion of any violence in patients with FEP was 34.5\% (95\% CI= 26.8-45.1) (Large \& Nielssen, 2011). Again, heterogeneity was high $\left(I^{2}=89.2\right)$ and there were differences in time frames (Large \& Nielssen, 2011).

\section{Child psychiatric facilities}

The prevalence of aggressive incidents during admission varies from $21.7 \%$ to $50 \%$ of all patients within child and adolescent psychiatric facilities (Crocker, Stargatt, \& Denton, 2010; Dean et al., 2008; Phillips, Stargatt, \& Fisher, 2011; Ryan, Hart, Messick, Aaron, \& Burnette, 2004; van Kessel, Milne, Hunt, \& Reed, 2012). Again, there are differences in measures and the length of hospitalization varies among facilities.

\section{Risk factors}

Aggression within psychiatric disorders is complex and multifaceted and does not originate from a single disorder, symptom or trigger. However, numerous studies have identified risk factors that are associated with aggressive behaviour. Identification of risk factors contributes to the understanding of aggression and enables early recognition of patients at risk.

When discussing risk factors, the growing amount of research on the biological explanations for aggressive behaviour should also be considered. It has been argued that aggression in humans is heritable, but its genetic basis remains to be found (Odintsova et al., 2019). Research has suggested that individuals who exhibit antisocial or criminal behaviour tend to have reduced brain volumes, as well as impaired functioning and connectivity in areas related to executive functions, emotion regulation, decisionmaking and morality, and have increased volumes in the reward regions of the brain (Ling, Umbach, \& Raine, 2019).These could be important findings for future treatment options. However, aggression cannot be explained by biology solely.

Some of the risk factors associated with aggressive behaviour in patients with psychiatric disorders are: previous aggression, male sex, socioeconomic deprivation, physical and sexual adversity during childhood, difficulties in social relationships 
during the stages of childhood, suicidality, impulsiveness, excitement, symptoms of mania, substance abuse, treatment non adherence, lack of insight and positive symptoms (i.e., symptoms that are usually not present but occur throughout different psychiatric illnesses, such as delusions and hallucinations) (Arango, 2000; Arango, Calcedo Barba, Gonzalez, \& Calcedo Ordonez, 1999; Bobes, Fillat, \& Arango, 2009; Bosqui et al., 2014; Fazel et al., 2009; Fresán et al., 2004; Hodgins \& Klein, 2017; Large \& Nielssen, 2011; Moulin, Baumann, et al., 2018; Moulin, Golay, et al., 2018; Swanson et al., 2006; Volavka et al., 2016; Witt, Hawton, \& Fazel, 2014; Witt et al., 2013).

Substance abuse is an important risk factor. A meta-analysis found that the risk for aggression in patients with schizophrenia is similar to the risk of patients who are not diagnosed with schizophrenia but do have substance abuse disorders, indicating that most of the excessive risk is mediated by substance abuse (Fazel et al., 2009). This is an interesting finding as this indicates that aggressive behaviour is linked to substance abuse, regardless of the presence of a psychotic disorder. Substance abuse has consistently been associated with aggressive behaviour, but for most other risk factors, there are differences between studies.

Risk factors are most useful in clinical practice if these are causal and modifiable and thereby give some indication for clinicians to reduce the risk. However, the relation between a risk factor and aggression is not always straightforward and risk factors differ between patients and even within patients over time. Mental health care workers should be aware of the known risk factors for aggressive behaviour, but consider them within the specific context.

\section{Clinical risk management}

Prevention and management of aggression is an important concern within mental health care facilities, specifically within inpatient facilities. These wards are a somewhat unnatural environment, often with severely ill patients that are frightened, suspicious or confused. Multidisciplinary efforts should focus on ensuring a safe ward environment, including clinical risk management strategies. Risk management includes strategies to prevent aggressive behaviour or to reduce its consequences. Risk management is a continuous process consisting of three main components: risk assessment, prevention and management of aggressive behaviour, and evaluation of incidents.

\section{Risk assessment}

Risk assessment can be conducted in a structured or a non-structured matter. Nonstructured, the professional assesses the risk based on experience and known risk factors. For a structured risk assessment, instruments can be used. 
Risk assessment instruments quantify the risk for aggressive behaviour for the shortor the long term. Risk assessment instruments were originally developed in forensic psychiatry to determine the long-term risk for violent behaviour (Webster \& Hucker, 2007) but are now used throughout psychiatric facilities. New instruments were developed to assess the short-term risk for aggressive behaviour by incorporating dynamic items that are important for clinical risk assessment (McDermott \& Holoyda, 2014). There is a growing amount of evidence that risk assessment instruments are useful in clinical psychiatry (Anderson \& Jenson, 2019; van de Sande et al., 2013).

One example of a short-term risk assessment instrument is the Bröset Violence Checklist (BVC (Almvik \& Woods, 1999)). The BVC is widely used in clinical psychiatry to predict physical aggression within the next 24 hours. The BVC incorporates six items that are scored every nursing shift (present or absent): confused, irritable, boisterous, verbal threats, physical threats, attacking objects (Almvik \& Woods, 1999). The BVC was developed for adult psychiatry. Few studies focus on risk assessment in child- and adolescent psychiatry. One instrument has been developed for short-term risk assessment: the Brief Rating of Aggression by Children and Adolescents (BRACHA (Barzman et al., 2011)).

There is evidence that the BVC assists in clinical risk assessments, specifically on acute wards (Abderhalden et al., 2004; Sarver, Radziewicz, Coyne, Colon, \& Mantz, 2019; Woods \& Almvik, 2002) and there are suggestions that risk assessment contributes to a reduction of aggression and coercive interventions (Hvidhjelm et al., 2016; van de Sande et al., 2011). There is some discussion on the latter (Large \& Ryan, 2015) but risk assessment instruments are recommended in clinical guidelines (Inspectorate of Health, 2011; National Collaborating Centre for Mental Health, 2015). Risk assessment instruments should be seen as an addition to the clinical judgement of the professional. Thereby, risk assessment instruments assist in structured thinking about risks and contribute to an early detection of aggression.

\section{Prevention and management of aggressive behaviour}

Following the risk assessment outcome, efforts should be made to reduce the risk and prevent future aggressive incidents. Patients should not be labelled as "aggressive" or "non-aggressive" but the results from the risk assessment should lead to a joint effort of clinicians and nurses to incorporate the risk in treatment planning. This could include a range of different therapeutic interventions, in line with the preferences of the patients, such as useful activities, sports, involvement of family, "as needed" medication, etc. Standard interventions should be adapted according to the patients' needs. However, the biggest challenge in clinical risk assessment is not the actual assessment but the use of subsequent interventions. One study found that interventions derived from risk assessment are frequently psychopharmacological or coercive, but 
not focused on prevention (Kaunomaki et al., 2017). The use of non-restrictive deescalation techniques is considered a best practice, although there is limited evidence for the effectiveness of those techniques (Du et al., 2017; Gaynes et al., 2017; Hallett \& Dickens, 2017). De-escalation techniques are aimed at preventing incidents in situations of emerging aggression. These techniques can be incorporated in personal de-escalation plans. These plans consist of personal warning signs, triggers and preferred interventions for specific patients. De-escalation plans should be made with patients and relatives (Kontio, Lantta, Anttila, Kauppi, \& Valimaki, 2017) but findings suggest the involvement of patients in these plans is low (40\%) (Hallett, Huber, Sixsmith, \& Dickens, 2016).

Next to preventive interventions, there are pharmacological options for the management of aggression. First, "as needed" (or Pro Re Nata (PRN)) oral sedatives are frequently administered to prevent upcoming escalations. Specifically benzodiazepines are used to reduce anger and fear. However, a systematic review concludes the evidence on the effectiveness of benzodiazepines for psychosis-induced aggression is of low quality, with mostly sponsored studies, low sample sizes, and risk for bias (Zaman et al., 2018). Second, when oral "as needed" medication is not an option or immediate sedation is needed, rapid tranquillisation (or "chemical restraint") could be considered. Rapid tranquillisation is the use of medication, voluntarily or coercive, mostly by intramuscular injections, during an aggressive episode (National Collaborating Centre for Mental Health, 2015). Currently, haloperidol is recommended for rapid tranquillisation, but again with limited evidence (de Almeida et al., 2017; MuirCochrane et al., 2019). Enforced medication, like other coercive measures, is used only as a last resort. Third, for the pharmacological treatment of aggression in patients with psychotic disorders, antipsychotics are preferred over benzodiazepines as these address the underlying psychopathology (Volavka \& Citrome, 2018). Clozapine appears to be most effective against hostility in patients with psychotic disorders (Frogley, Taylor, Dickens, \& Picchioni, 2012) but this superiority is not well documented and the mechanisms are currently not completely clear. Moreover, as treatment non adherence is a risk factor for aggression, long acting injectable antipsychotics (LAI, or depot medication) may have advantages over oral antipsychotics (Arango, Bombin, GonzalezSalvador, Garcia-Cabeza, \& Bobes, 2006; Fazel, Zetterqvist, Larsson, Långström, \& Lichtenstein, 2014; Topiwala \& Fazel, 2011). However, the difficulty in titration and low tolerability for LAI in some patients should be considered (Fazel, Zetterqvist, Larsson, Langstrom, \& Lichtenstein, 2014).

\section{Evaluation of aggressive incidents}

Evaluation of aggression should take place after each incident and in a structural matter. First, there should be some kind of aftercare for both staff and patient, immediately after 
the incident. Post-incident debriefing allows staff to evaluate the incident. Evaluation with patients is important as they could forget what happened during incidents and feel like they are treated unfairly (Richter \& Whittington, 2006). Patients could perceive incidents as less severe compared with nurses (Vermeulen et al., 2019). Joint evaluation, including a reflection on antecedents and triggers, gives both patient and staff indications to prevent future incidents. This can, for example, be incorporated in the patients' personal risk management or de-escalation plan. Furthermore, debriefing incidents between patients and staff allows for mutual understanding and, if needed, reconstruction of the therapeutic relationship.

Second, for the structural evaluation of aggression within psychiatric facilities, incident report forms, or aggression evaluation tools, are important. With use of these instruments, aggressive incidents can be documented. One widely used instrument, that includes an objective severity scale, is the Staff Observation Aggression ScaleRevised (SOAS-R (Nijman \& Palmstierna, 2002)). Structured data collection allows for clinical evaluation of aggression and can be used for research purposes. During regular ward meetings, data derived from incident reports, in combination with data on coercive interventions, can help to reveal patterns. For example, if there are specific time frames or antecedents prior to the incident, this can be an incentment for a change in policy or organizational factors.

\section{Contextual factors}

While clinical risk management is mostly focussed on internal factors, context may be equally important. Nijman et al. describe how aggression emerges from patient related factors, ward variables and staff variables and state that even when psychopathology is the primary cause of violent behaviour, the contribution of environmental- and communication stressors should not be ruled out (Nijman, 2002; Nijman, aCampo, Ravelli, \& Merckelbach, 1999). More recently, Safewards has been introduced as a model to reduce conflict and containment management on inpatient wards, focusing on all types of conflict and not only aggression (Bowers, 2014). The following section discusses four contextual concerns: communication and interaction, staff training, ward features, and user experiences.

\section{Communication and interaction}

Traditionally, communication, the development of rapport, and establishing therapeutic relationships are essential in psychiatric nursing. This is not different when it comes to the prevention and management of aggression. Aggression is interpersonal and the antecedents prior to aggressive behaviour are often within patient-staff relations, such 
as restrictions or the patient being denied something (Nijman, Allertz, Merckelbach, A Campo, \& Ravelli, 1997; Papadopoulos et al., 2012). Qualitative findings have indicated that patients experience a custodial rather than caring approach contributes to aggression (Gudde, Olso, Whittington, \& Vatne, 2015) and feel a need for humane and interactive nursing in the prevention of aggression (Kontio et al., 2014).

"The first five minutes" is an intervention based on the assumption that the first encounter with the patient determines the quality of the relationship and was originally developed to prevent seclusion (Welleman, Stringer, Landeweer, \& Gijsbers van Wijk, 2011). This intervention is described in a protocol. However, an analysis from an ethical perspective concluded that the application of the intervention implies more than following the steps of the protocol but requires a new way of thinking and acting (Voskes, Kemper, Landeweer, \& Widdershoven, 2014). The quality of contact and communication cannot be captured in a list or protocol. Wards should have an empathic, attentive and recovery-oriented atmosphere that allows for meaningful communications between patients and health care workers. Health care workers should focus on establishing and keeping the therapeutic relationship with the patient intact.

In patients with psychotic disorders, communication is often complicated due to symptoms such as delusions and fear. Specifically when aggression is psychosisdriven, it is of even greater importance to make contact with the patient and establish rapport. One approach that can help in the communication with patients with psychotic disorders is Listen, Empathize, Agree, Partner (LEAP (Amador \& Johanson, 2000)). The LEAP approach is developed for patients with a lack of insight and can help patients to accept treatment (Paillot, 2009). As these principles are based on understanding, creating and keeping contact with patients, these principles could also help during situations of emerging aggression.

\section{Staff training}

Staff should be trained in the prevention and management of aggressive behaviours. Most nurses and clinicians receive some kind of training within mental health care facilities. These training courses are recommended as best practice (National Collaborating Centre for Mental Health, 2015) although there is limited evidence for their effectiveness (Price, Baker, Bee, \& Lovell, 2015). Ideally, training courses should be focussed on prevention, risk management and de-escalation but also include the safe application of restrictive interventions, such as seclusion and restraint, and breakaway techniques. Training-on-the-job could be essential in bringing the techniques to clinical practice. 


\section{Ward features}

Within inpatient facilities, several ward features can affect the occurrence of aggression. Multiple studies found a reduction in aggressive incidents and seclusions following improvements in the ward design or interior (Jenkins, Dye, \& Foy, 2015; Ulrich, Bogren, Gardiner, \& Lundin, 2018; van der Schaaf, Dusseldorp, Keuning, Janssen, \& Noorthoorn, 2013). Other ward features associated with aggression include: poor staffing levels, boredom, lack of privacy and freedom, vague ward policies and ward turmoil (Duxbury, Bjorkdahl, \& Johnson, 2006; Panagiotou, Mafreda, Moustikiadis, \& Prezerakos, 2019).

\section{User experiences}

Patients and health care workers experience aggressive behaviour differently. User perspectives can help in bringing these experiences closer together. A number of qualitative studies describe triggers and perspectives of patients about aggression (Gudde et al., 2015; Price et al., 2018). User experiences should be collected in a routine matter and incorporated in the aggression policies. This can improve the collaboration between staff and patients and contribute to a joint effort towards more safe wards.

\section{Gaps in current literature}

This chapter provided an introduction on aggression within psychiatric practice. We described a number of research papers that currently support clinical practice. However, many clinical decisions considering aggressive behaviour are still not evidence-based. For one, while some evidence about warning signs in adult psychiatry exists, little is known about warning signs in child psychiatry. This hampers efforts directed at early recognition in a population where aggression is a complicated problem. Second, there is a need for reliable and clinically useful risk assessment instruments in adult psychiatry. Some currently used instruments have low clinical utility or incorporate few risk factors that give implications for prevention. This hinders effective use of these instruments. Third, more evidence is needed about incidence and risk factors for aggressive behaviour as there are many methodological differences between studies and many studies are retrospective. Incidence data and risk factors could help in unravelling the complex phenomenon of aggression. Finally, there is insufficient evidence for some frequently used pharmacological interventions, such as the antipsychotic treatment of FEP patients with aggressive behaviour. Moreover, while clozapine appears to be the most effective antipsychotic agent against persistent hostile or aggressive behaviour in patients with psychotic disorders, this is not well documented and the underlying 
mechanisms are not clear. These are some of the issues that currently hinder the effective and evidence based assessment and management of aggressive behaviour. While aggression is a complex phenomenon and there are inherent methodological difficulties in studying this subject, more research is needed to support clinical practice.

\section{Aim and outline}

The aim of this thesis is to contribute to current knowledge about aggression in psychiatry with clinically relevant results and insights. The focus is on three main themes:

- Prevention: warning signs and risk assessment

- Epidemiology: incidence, prevalence and risk factors for aggressive behaviour

- Treatment: efficacy of antipsychotics on aggressive behaviour

These themes are addressed in the following chapters (Table 1). Chapter 2 aims to identify the most frequently observed warning signs prior to aggressive incidents in child psychiatry. The third chapter is aimed at the clinimetric properties of a routine outcome instrument that can assist in the assessment of the short-term risk for aggression and other adverse outcomes. The fourth chapter is a survival analysis of a large cohort study with the aim to investigate the incidence and risk factors of hostile and aggressive behaviour. The fifth chapter is aimed at identifying risk factors and the effectivity of antipsychotic agents on hostile and aggressive behaviour in FEP. The sixth chapter is a meta-analysis that compares the effect of typical versus atypical antipsychotics on hostility. The results of these research chapters are summarized in chapter 7 . Finally, in chapter 8 , the results and clinical implications are discussed and directions for future research are suggested.

\section{Table 1. Outline of the research chapters}

\begin{tabular}{|c|c|c|c|}
\hline Theme & Chapter & Scope & Population \\
\hline \multirow[t]{2}{*}{ Prevention } & 2 & $\begin{array}{l}\text { Warning signs prior to aggressive } \\
\text { behaviour }\end{array}$ & Child inpatients \\
\hline & 3 & $\begin{array}{l}\text { Clinical risk assessment: interrater } \\
\text { reliability and clinical utility }\end{array}$ & $\begin{array}{l}\text { Adult and adolescent in- and } \\
\text { outpatients }\end{array}$ \\
\hline \multirow[t]{2}{*}{ Epidemiology } & 4 & $\begin{array}{l}\text { Incidence and risk factors of aggressive } \\
\text { behaviour }\end{array}$ & $\begin{array}{l}\text { Patients with psychotic } \\
\text { disorders }\end{array}$ \\
\hline & 5 & $\begin{array}{l}\text { Prevalence and risk factors of hostile and } \\
\text { aggressive behaviour }\end{array}$ & First episode psychosis \\
\hline \multirow[t]{2}{*}{ Treatment } & 5 & $\begin{array}{l}\text { Effectivity of amisulpride versus } \\
\text { olanzapine on hostility during the first } \\
\text { weeks of treatment }\end{array}$ & First episode psychosis \\
\hline & 6 & $\begin{array}{l}\text { Effectivity of typical versus atypical } \\
\text { antipsychotics on hostility }\end{array}$ & $\begin{array}{l}\text { Patients with psychotic } \\
\text { disorders }\end{array}$ \\
\hline
\end{tabular}





\section{CHAPTER 2}

Warning signs prior to aggressive behaviour in child psychiatric units

Margo Faay

Marije Valenkamp

Henk Nijman

Archives of Psychiatric Nursing (2017), 31(1), 43-47 
This study aims at detecting and categorizing early warning signs of aggressive behaviour in child psychiatric units. We analysed 575 violent incident report forms and developed a coding scheme consisting of 16 warning signs. From the 575 incident report forms, a total of 1087 signs were coded. Most common warning signs were "restlessness" (21.2\%), "not listening" (15.2\%) and "anger" (9.8\%). These were also the most prevalent warning signs for the severe incidents. Although warning signs differ for each individual child, this study indicates that there are common warning signs for imminent aggressive incidents in child psychiatric facilities.

\section{ABSTRACT}




\section{Introduction}

Severe aggression is one of the most common issues for children to be referred to a psychiatric unit and is subsequently a major issue within these units (Blake \& Hamrin, 2007; Valenkamp, 2011). Studies found that between $21.7 \%$ and $59 \%$ of children show one or more aggressive incident during their stay on a psychiatric unit (Crocker et al., 2010; Dean et al., 2008; Phillips et al., 2011; Ryan et al., 2004; van Kessel et al., 2012). It should be noted that due to differences in definitions and measures, it is difficult to determine the frequency and make comparisons between different units, institutions or countries.

Aggression during hospitalization has a major impact on both staff and patients. Dean, Gibbon, McDermott, Davidson, and Scott (2010) concluded that aggression has a physical, emotional and professional impact on staff and could impair their therapeutic capacity. Aggression is also one of the most common motives for the use of restrictive interventions by staff such as seclusion and restraint (Sukhodolsky, Cardona, \& Martin, 2005).

De Hert, Dirix, Demunter, and Correl (2011) conducted a literature review including 7 publications and found that $26 \%$ of hospitalized children and adolescents under 21 years of age are secluded and 29\% experience a physical or mechanical restraint at least once during their stay in child and adolescent psychiatric facilities. Restrictive interventions are often considered effective to reduce the immediate risk of harm to patient and others. Finke (2001) however, found no scientific evidence considering the effectiveness of seclusion in the psychiatric care of children and concluded that seclusion is not therapeutic but rather harmful for the aggressive patients. Moreover, children who witness the seclusion or physical restriction of a group member may also be traumatized by the event. Another study found that staff experienced anger, distress and anxiety as a response to the use of restraint (Sequeira, 2004).

With this paper, we hope to contribute to a reduction of aggressive incidents and subsequent negative consequences by searching for ways to predict aggressive incidents in child psychiatric units, with the aim of providing staff members with more proactive options to prevent imminent escalations. For the purpose of the current study we use the definition of aggression from Morrison (1990), which formulates that aggression is: "any verbal, non-verbal, or physical behaviour that is threatening (to self, others or property), and/or physical behaviour that actually harms (to self, others, or property)."

In the prediction of aggressive incidents, risk factors, provocations and early warning signs are essential. This paper focuses on the early warning signs. 


\section{Individual risk factors, provocations and early warning signs}

Some static patient characteristics can be associated with higher levels of inpatient aggression. Crocker et al. (2010) found that children with a disruptive behaviour disorder were four times more likely to engage in more severe aggression. Philips et al. (2011) found similar results and stated that a history of trauma is also predictive for violent incidents. Hage, van Meijel, Fluttert, and Berden (2009) conducted a literature review and found that in adolescent psychiatric settings below- average levels of intellectual functioning, a diagnosis of ADHD, male gender, oppositional defiant disorder, conduct disorder, several personality traits and a history of prior aggressive behaviour are static risk factors for aggressive incidents in the units. Barzman et al. (2011) developed an actuarial method that may help clinicians to rapidly assess the risk for aggressive behaviour by hospitalized children and adolescents. The BRACHA includes potential historical predictors of aggression such as abuse in the past, previous psychiatric hospitalization and recent or past aggressive behaviour or violent incidents (Barzman et al., 2011). Aggressive incidents with children at risk however, usually do not occur unprovoked and out of the blue. Valenkamp (2011) found that in $94.7 \%$ of 1029 inpatient aggressive incidents in children (4-12 years of age), a detectable provocation preceded the incident. In more than $50 \%$ of these cases, the provocation concerned limit setting or correction of the child by staff. Similar results were found by Ryan et al. (2004) in an inpatient population of youths (6-17 years of age). From a total of 215 incidents, in $94 \%$ a provocation was reported. The most common provocation $(68 \%)$ was a request, direction or command from the staff that could be perceived as coercive or limit setting by the patient (Ryan et al., 2004).

Although risk factors and provocations can be helpful in the assessment of the risk for aggression, risk factors in terms of patient's behaviour prior to aggressive incidents are more closely and directly associated with incidents compared to static risk factors and provocations (Steinert, 2006). Inpatient aggression is usually preceded by observable behaviours such as non-violent agitation (Hankin, Bronstone, \& Koran, 2011). These observable behaviours or early warning signs enables staff and/or patients to anticipate on changes in behaviour in an attempt to minimize escalation with use of tailored preventive interventions (Faay, van de Sande, Gooskens, \& Hafsteinsdottir, 2013; van de Sande et al., 2013). Unfortunately, evidence considering warning signs prior to aggressive incidents in child psychiatry is scarce.

In adult psychiatry, research considering early warning signs of aggressive incidents has more of a tradition. Almvik, Woods, and Rasmussen (1999) developed the Broset Violence Checklist (BVC) which incorporates six behavioural characteristics: confusion, irritability, boisterousness, verbal threats, physical threats and attacks against objects. Each item can be scored 0 or 1 and a total score of 2 or higher indicates a markedly increased risk of violence within 24 hours (Almvik \& Woods, 2003). The 
instrument has been translated in several languages, has good psychometric properties and is considered short and easy to use in daily clinical practice (Almvik \& Woods, 1999, 2003; Clarke, Brown, \& Griffith, 2010). The BVC enables staff to objectively monitor patient behaviour on a daily basis and anticipate on the first behavioural changes. One study found a reduction of $41 \%$ in aggressive incidents and a reduction of $27 \%$ in the use of coercive measures by using the BVC during the first days of treatment (Abderhalden et al., 2008). Another study using multiple risk assessment instruments, among which the BVC, also found a significant reduction of aggressive incidents and time spent in seclusion (van de Sande et al., 2011).

Unfortunately, the BVC has never been tested in a population of children as far as we know. We consider the concept of aggression in children to be different from adult populations as evidence supports an increase of aggression during specific developmental periods (Reebye, 2005). Some forms of aggression are considered as normal behaviour in children as they learn to control their aggression during their development (Blake \& Hamrin, 2007). For example, two of the items on the BVC, "boisterousness" and "attacks on objects," are more common behaviours for children compared with adults and could be considered age-appropriate to a certain extent. Therefore we can learn from the extensive experience and research in adult populations but we also need to gather knowledge on the specific warning signs for aggression in children's populations.

\section{Study aim}

The aim of this study is to determine the most prevalent early warning signs prior to aggressive incidents in a sample of children from 4 to 12 years of age in clinical and semi-clinical psychiatric facilities.

\section{Methods}

\section{Participants \& measures}

The Proactive monitoring of Aggression in Children Tool (Valenkamp,Verheij, Nijman, Palmstierna, \& Bjorkly, 2013; Valenkamp, Verheij, Nijman, Palmstierna, \& Bjørkly, 2009) was developed for staff to report aggressive incidents. ProACT is based on the staff observation aggression scale- revised (SOAS-R (Nijman, Muris, et al., 1999)) and the report form for aggressive episodes (REFA (Bjorkly, 1996)) and adapted for use in a child inpatient population. The ProACT consists of several categories: provocation, means (aggressive behaviours of the child), target, consequences and measures to stop the aggression or to prevent further escalation. Each of the categories consist of one 
multiple choice question (for example in category provocation: "what trigger was there just before the aggression started?") in which respondents are asked to select the best possible answer out of the choices from a list (for example in category provocation: "unexpected situation," "pressure to do a certain obligation/task" or "child was bored" etc.). In the current study the one open-ended question (free text) in the ProACT form about early warning signs was used: "which warning signs did you observe?"

Data that was used for the current study were collected for another study that did not use the data on warning signs (Valenkamp, 2011). Data were collected from April 2009 until June 2010 in 26 inpatients units within 4 child psychiatric settings and 2 child welfare services for children aged 4-12 years in the Netherlands. 236 inpatient children were admitted on these units during the study. Of these children, 131 (56.4\%) were involved in one or more aggressive incidents as documented with the ProACT. The children participated on average 12.3 weeks (SD 4.0) in the study. The demographic data of the children involved in the study are displayed in Table 1.

The local medical ethics committee gave permission for the original study from which we retrieved this data (Valenkamp, 2011). Because the original study was completely observational and non-intrusive the medical ethics committee decided that no active permission was needed from the patient or parents. However, all parents received a letter explaining the study including an opt out choice. With this opt out procedure, parents could object if they did not want the data of their child to be used for the study.

\section{Data analysis}

A total of 1068 incidents were reported for the 131 children. The mean number of incidents per child is 8.2. On 575 incident report forms (53.8\%) one or more warning signs were entered. Of these 575 forms, 62 incidents were categorized as severe $(10.8 \%)$, meaning there were physical consequences for staff, varying from pain to the need for treatment. All 575 incident report forms were coded to gain insight in the most common warning signs. To ensure a valid and reliable coding process, we followed 6 steps:

1. Coding. All descriptions from the 575 incident records were coded by the first author of this paper. These warning signs were then reviewed by the first two authors to ensure signs were clearly formulated and not multi interpretable. This initial open coding process resulted in a coding scheme of 34 signs with short descriptions and 4 broad categories to cluster the 34 signs.

2. Expert review round I. The initial coding scheme was reviewed by 29 experts from four child psychiatric institutions: 20 group workers (nurses, advanced nurse 
Table 1: Demographic data of the children with aggressive incident reports

\begin{tabular}{|c|c|}
\hline & $\begin{array}{c}\text { Total }(\mathbf{n = 1 3 1 )} \\
\text { N (\%) }\end{array}$ \\
\hline Number of incident reports per child & $\begin{array}{c}27(20.6) \\
46(35.1) \\
27(20.6) \\
11(8.4) \\
8(6.1) \\
12(9.2)\end{array}$ \\
\hline $\begin{array}{l}<6 \\
6-9 \\
>9\end{array}$ & $\begin{array}{l}22(16.8) \\
53(40.5) \\
41(31.3)\end{array}$ \\
\hline $\begin{array}{r}\text { Male } \\
\text { Female }\end{array}$ & $\begin{array}{c}103(78.6) \\
20(15.3)\end{array}$ \\
\hline Attention-deficit/hyperactivity disorder & $51(38.9)$ \\
\hline Pervasive Development Disorder & $43(32.8)$ \\
\hline Oppositional defiant disorder & $35(26.7)$ \\
\hline Mental retardation & $21(16.0)$ \\
\hline Reactive attachment disorder & $20(15.3)$ \\
\hline Other & $12(9.1)$ \\
\hline Conduct disorder & $11(8.4)$ \\
\hline Mood disorder & $5(3.8)$ \\
\hline Anxiety disorder & $4(3.1)$ \\
\hline Adjustment Disorder & $2(1.5)$ \\
\hline
\end{tabular}

practitioners and other professionals such as pedagogical workers), 2 psychiatrists, 1 developmental psychologist and 2 nursing policy advisors. Experts were invited to write down their feedback about the formulations of the signs on the initial coding scheme. Feedback was used to adjust the signs and descriptions. This resulted in a new version of the coding scheme including 44 signs separated into 4 broad categories.

3. Intercoder agreement test I. A total of 115 randomly selected incident records $(20 \%)$ were scored independently by author 1 and 2 using the 44 signs coding system. In total, 380 signs were coded. In 282 signs (74.2\%) both researchers assigned the same code. Subsequently, the differences were analysed and signs with the weakest agreement were more clearly defined. Some new signs emerged. After this modification, the coding scheme consisted of 48 signs. 
4. Expert review round II. From the 29 initial experts, a random selection of 5 experts was asked to review the new coding scheme. Their suggestions were used to modify the scheme. Seven signs were deleted. For example because there was too much resemblance with other signs according to the experts. This resulted in a coding scheme of 41 signs divided into 4 categories.

5. Intercoder agreement test II.The second intercoder agreement test was conducted with 127 (22\%) of the 575 incident report forms (different than the 115 forms used in the first intercoder agreement test). A total of 248 signs were coded of which $182(73.4 \%)$ both researchers assigned the same code. To assess the intercoder reliability more specifically, Cohen's kappa was calculated for all 41 signs (Table 2).

6. Final coding. All 575 incident reports were coded by author 1 according to the 41 signs. To define an optimal coding set of frequently observed warning signs, we used two criteria. First, signs with low or moderate interrater agreement (kappa $<0.60$ according to the criteria of Landis \& Koch, 1977) were removed. Second, signs scored less than 10 times were excluded. The final coding scheme is described in the result section.

After the forms were coded using this final coding scheme, a chi-square analysis was conducted to test if certain warning signs are significantly associated with the 62 most severe incidents with physical consequences.

\section{Results}

\section{Construction of the final coding scheme}

According to the criteria described in step 6, 6 signs with moderate or less kappa $(<0.60)$ (Landis \& Koch, 1977) were removed. Second, 16 signs that were scored less than 10 times in the final coding were excluded. Finally, both "angry" and "restlessness," that were first separated in three signs ("verbal," "non-verbal" and "not otherwise specified") were clustered, reducing the number of signs with four.

The signs that were excluded due to this process were incorporated in a new category, "other." Therefore, the final coding scheme consists of 16 signs and 4 categories (Table 2). 
Table 2: Final coding scheme with Cohen's Kappa for intercoder agreement and Chi square for severe versus non severe incidents

\begin{tabular}{|c|c|c|c|c|}
\hline Sign & Description & $\mathbf{N}(\%)$ & $\begin{array}{l}\text { Cohen's } \\
\text { Kappa }\end{array}$ & $\begin{array}{l}\text { Chi } \\
\text { square }\end{array}$ \\
\hline \multicolumn{5}{|c|}{ Category: Negative behavior } \\
\hline Grumble & Grumbling, complaining, whining, protesting. & $65(6.0)$ & .90 & 2.91 \\
\hline Rude & Verbal or nonverbal rude or cheeky expressions. & $53(4.9)$ & 1.0 & 1.82 \\
\hline $\begin{array}{l}\text { Exploring the } \\
\text { edges }\end{array}$ & $\begin{array}{l}\text { Consciously challenging or provoking other } \\
\text { persons, without the intention of teasing. For } \\
\text { example: violating the rules, seeking attention or } \\
\text { triggering a response. }\end{array}$ & $85(7.8)$ & .72 & 0.40 \\
\hline Not listening & $\begin{array}{l}\text { Not responding to a correction of any kind, } \\
\text { refusing to cooperate, saying 'no' or refusing } \\
\text { something in any other manner. }\end{array}$ & $\begin{array}{c}165 \\
(15.2)\end{array}$ & .88 & 2.45 \\
\hline $\begin{array}{l}\text { Absconding/ } \\
\text { hiding }\end{array}$ & Running or walking away or hiding. & $23(2.1)$ & .92 & $4.75^{*}$ \\
\hline $\begin{array}{l}\text { Raising one's } \\
\text { voice }\end{array}$ & Speaking out, talking too loud (not: shouting). & $26(2.4)$ & 1.0 & 0.22 \\
\hline Scream & Screaming, yelling, shouting. & $25(2.3)$ & .83 & 0.76 \\
\hline $\begin{array}{l}\text { Unfocused } \\
\text { swearing }\end{array}$ & $\begin{array}{l}\text { Swearing, cursing or other abusive language, } \\
\text { without the meaning of hurting the other person } \\
\text { directly (which is considered violence). }\end{array}$ & $32(2.9)$ & .95 & 1.36 \\
\hline \multicolumn{5}{|c|}{ Category: Restlessness } \\
\hline $\begin{array}{l}\text { Making noises } \\
\text { - non verbal }\end{array}$ & $\begin{array}{l}\text { Making noises with objects, tapping or drumming } \\
\text { with feet or hands or stamping with feet on the } \\
\text { ground. }\end{array}$ & $20(1.8)$ & .80 & 2.00 \\
\hline $\begin{array}{l}\text { Unrest/ } \\
\text { restlessness }\end{array}$ & $\begin{array}{l}\text { Verbal unrest or motility. For example: walking } \\
\text { around, lying on the floor, not able to sit still, } \\
\text { talking too much, muttering, making noises with } \\
\text { mouth, asking questions or sighing. }\end{array}$ & $\begin{array}{c}230 \\
(21.2)\end{array}$ & .87 & $4.87 *$ \\
\hline \multicolumn{5}{|c|}{ Category: Emotion } \\
\hline Tense & Stressed, nervous. & $13(1.2)$ & .79 & 0.03 \\
\hline Irritated & Irritable, touchy, bad mood or testy. & $60(5.5)$ & .74 & 0.59 \\
\hline Anger & $\begin{array}{l}\text { Verbal (angry, agitated, surly) or non-verbal } \\
\text { expression of anger (angry facial expression } \\
\text { or other physical expression of anger. Not: } \\
\text { threatening behavior or damaging objects (this is } \\
\text { defined as aggression). }\end{array}$ & $106(9.8)$ & .72 & 0.31 \\
\hline Introverted & Not in contact with other persons. & $22(2.0)$ & .65 & 0.00 \\
\hline Exuberant & $\begin{array}{l}\text { Overly cheery, laughing too loud, acting clownish, } \\
\text { making jokes or giggling. }\end{array}$ & $20(1.8)$ & 1.0 & 0.39 \\
\hline \multicolumn{5}{|c|}{ Category: Other } \\
\hline Other & $\begin{array}{l}\text { All other signs, not belonging to one of the signs } \\
\text { above. }\end{array}$ & $\begin{array}{c}142 \\
(13.1)\end{array}$ & N/A & $4.44 * *$ \\
\hline
\end{tabular}

${ }^{*} p<0.03 ; * * p<0.05$ 


\section{Warning signs and frequencies}

Using the final coding scheme, a total of 1087 signs were coded from the 575 incident reports. In 345 (60\%) incident reports, two or more signs were reported. The mean number of signs within the incident reports was 1.9 .

The most common warning sign was "restlessness" which was mentioned in $230(21.2 \%)$ incident reports (Figure 1). Second was "not listening" with 165 (15.2\%) entries. Third was "anger" with 106 (9.8\%) notifications. Most prevalent category was "negative behaviour" which was scored 474 (43.6\%) times. Second category was "unrest" which was scored 250 (23\%) times and third was "emotion" with 221 (20.3\%) entries. The least frequent category was "other" which was scored 142 (13.1\%) times.

From the 62 severe incidents reports, 97 signs were coded. "Restlessness" was the most common warning sign prior to these incidents, followed by "not listening" and "anger" (Figure 1). The chi-square analysis showed that both "restlessness" $\left(X^{2}=\right.$ 4.748, $p<0.03)$ and "absconding/hiding" $\left(X^{2}=4.748, p<0.03\right)$ were significantly more often associated with the severe incidents, when compared to the warning signs that were connected to the aggressive incidents not having any physical consequences for victims (Table 2).

Figure 1: Most frequent warning signs prior to aggressive incidents: all incidents versus incidents with physical consequence.

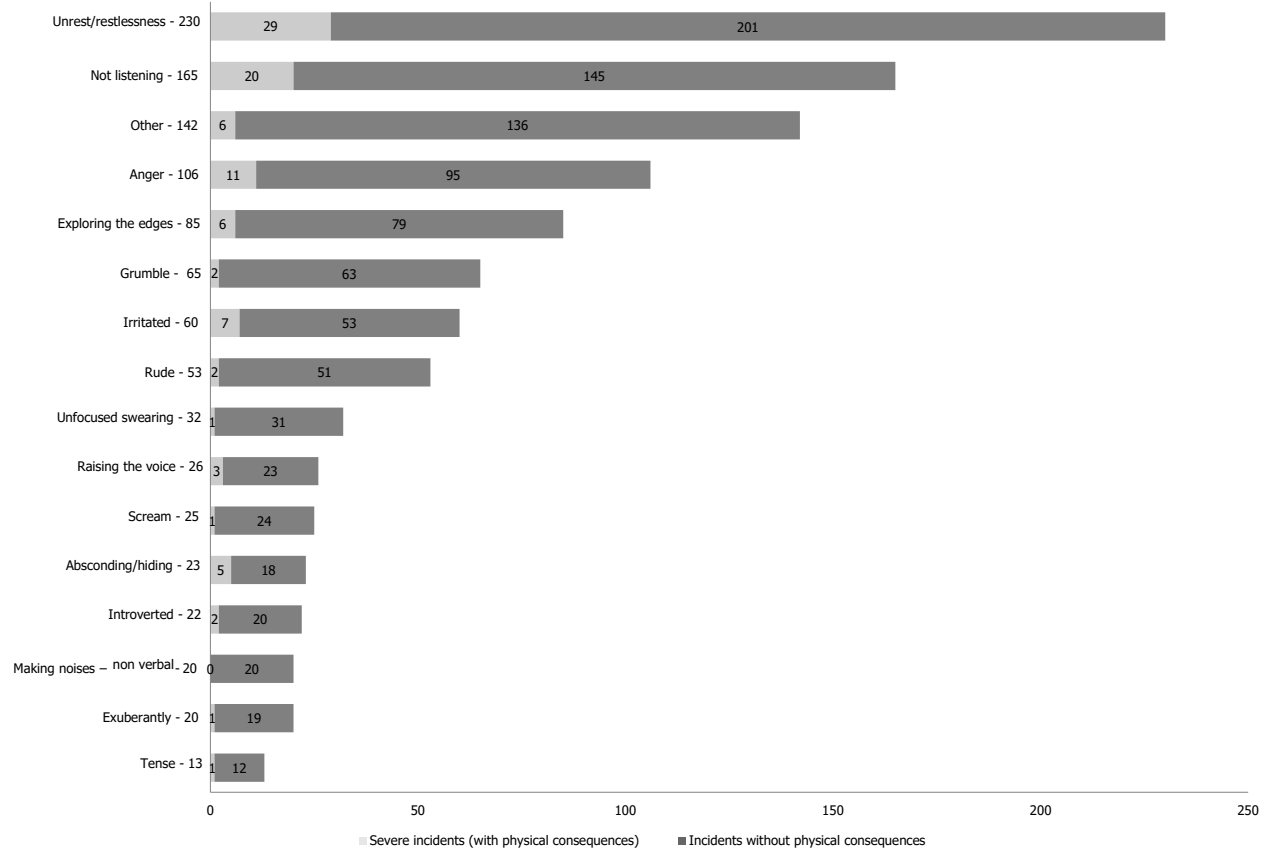




\section{Discussion}

\section{Statement of principle findings}

This study resulted in an overview of the 16 most prevalent warning signs that precede aggressive behaviour of young psychiatric patients. "Not listening," "restlessness" and "anger" are the most prevalent precursors of aggressive behaviour in child psychiatric units. Considering the severe incidents with physical consequences, for both "restlessness" and "absconding/hiding" we found a significant association. Restlessness could point at an accumulation of tension, resulting in more severe, especially physical, aggression. Absconding or hiding often requires physical intervention that could lead to physical injury.

\section{Limitations and strengths}

This study was conducted to gain a perspective on a subject that, as far as we know, has not been studied so far. This is the greatest strength of this study. Moreover, 575 incident reports were analysed and the data was retrieved from daily clinical practice. This may be a first step in the process to a professional and structured way of risk assessment in child psychiatric units.

However, there are several obvious limitations of this study. First, the data was retrieved for another study and the collection of warning signs was not the main focus of that study. This could explain why no warning sign was seen in $46.2 \%$ of the incidents. Second, there was only one source for the input of the warning signs, the ProACT.Third, only a part of the forms were scored by two researcher which was for the intercoder agreement test. Most forms were coded by one researcher only.

\section{Comparison with previous research}

Because this is, as far as we know, the first study specifically aiming at warning signs of aggression in children, we cannot compare our results with previous research. Nevertheless, there seem to be similarities between adult and child psychiatry as irritability and boisterousness are two important warning signs that are assessed in the BVC and also found (in somewhat different definitions) in the current study. As far as "not listening" is concerned, one can wonder whether this is a warning sign of aggression that is highly specific for children or whether this item could also be a warning sign in adult psychiatric patients. 


\section{Meaning of the study: implications for use in clinical practice}

There are several implications for use in clinical practice:

- The warning signs can possibly be helpful in daily practice for nurses to become more aware of changes in behaviours that can predict aggression.

- The signs can be integrated in aggression-evaluation forms, comparable with the ProACT. For example, a multiple choice option could be incorporated in the form, so that professionals choose the signs they observed before the incident. This may help to detect warning signs better next time the child is involved in an escalation and, moreover, data could be used for quantitative analyses for testing the scheme in the future.

- The warning signs can possibly be used in the making of personal de-escalation plans for children, giving both the professional, the parent and the child examples to help them think about which signs are applicable for the child.

- The signs could be helpful in the training of nursing students and professionals to observe and recognize early warning signs and to subsequently act on it.

- When the signs have been tested in clinical practice, perhaps a more structured tool can be developed, comparable with the BVC, for specific warning signals in children with psychiatric disorders. This might bring structured risk assessment in child psychiatry to a higher level.

\section{Implications for further research}

There are several steps that need to be undertaken to further complete our research. First, the clinical utility and interrater reliability of the scheme should be tested in a new study in clinical practice. Also, the scheme should be tested on its predictive capacity. It is likely that some of the signs in the scheme are behaviours that children with psychiatric problems often display without a subsequent aggressive incident. So, it is unsure whether the signs are specific enough to predict aggressive incidents. The presence of the signs could therefore be tested on inpatient facilities at different times during the day (at random). With this test, a selection of frequently observed signs could be distracted from the scheme that specifically precede aggressive incidents. Possibly, a predictive risk assessment instrument for child psychiatric units could be constructed in this way. Finally, aggressive behaviour of children could have many reasons and origins. To gain more insight, the warning signs could be compared with different patient characteristics. For example, are there certain diagnoses that are more closely related to some of the warning signs, and do boys display different behaviours than girls preceding aggression? 


\section{Conclusion}

This study found that "restlessness," "not listening" and "anger" are the most frequently observed warning signs after analysing 575 incident reports. Although warning signs may differ for each individual child, this study indicates that there are common warning signs. Future research should distinguish the warning signs relevant to different patient profiles. The results of this study could be the start of a more structured way to detect early warning signs in child psychiatry. Detection may be a first step in reducing aggressive incidents and thereby, this scheme with 16 warning signs could contribute to a safer environment in child psychiatry for children, family members and professionals. 



\section{CHAPTER 3}

\section{Kennedy Axis V: clinimetric properties assessed by mental health nurses}

Margo Faay

Roland van de Sande

Floor Gooskens

Thóra Hafsteinsdóttir

International Journal of Mental Health Nursing (2012), 22(5), 453-464 
The Kennedy Axis V is a routine outcome measurement instrument which can assist the assessment of the short-term risk for violence and other adverse patient outcomes. The purpose of this study was to evaluate the interrater reliability and clinical utility of the instrument when used by mental health nurses in daily care of patients with mental illness. This cross-sectional study was conducted in inpatient and outpatient adult psychiatric care units and in one adolescent inpatient unit at a university hospital in the Netherlands. Interrater reliability was measured based on the independent scores of two different nurses for the same patients. The clinical utility of the instrument was evaluated by means of a clinical utility questionnaire. To gain a deeper understanding of rating difficulties at the adolescent unit, additional data were collected in two focus group interviews. The overall results revealed a substantial level of agreement between nurses (intraclass correlation coefficient and Pearson 0.79). Some rating challenges were identified, including difficulties with scoring the instrument and using tailormade interventions related to the scores. These challenges can be resolved using refined training and implementation strategies. When the Kennedy Axis V is accompanied by a solid implementation strategy in adult mental health care, the instrument can be used for short-term risk assessment and thereby contribute in efforts to reduce violence, suicide, self-harm, severe self-neglect, and enhanced objectivity in clinical decisionmaking.

\section{ABSTRACT}




\section{Introduction}

Early recognition of emerging agitation is crucial to prevent or minimize severe violent incidents to self or others at acute psychiatric units. Violence often results in seclusion in some European countries (Janssen et al., 2011; Kaltiala-Heino, Tuohimaki, Korkeila, \& Lehtinen, 2003; Keski-Valkama et al., 2010). Various studies reveal that seclusion rates differ substantially regionally in the same type of clinical populations. The prevalence of seclusion varies 20-74\% (Janssen et al., 2011; Keski-Valkama et al., 2010; Tunde-Ayinmode \& Little, 2004). Worldwide, many psychiatric hospitals are involved in seclusion and restraint reduction programs (Husum, Bjorngaard, Finset, \& Ruud, 2010; Hyde, Fulbrook, Fenton, \& Kilshaw, 2009; Steinert et al., 2010). The prevention of violent incidents is crucial as they are highly associated with the start of seclusion episodes (Foster et al., 2007). Therefore, early recognition of increased agitation and deterioration needs constant attention to enhance proactive risk management strategies. Risk assessment instruments are important aids to facilitate effective risk management in clinical psychiatry (Webster \& Hucker, 2007).

Risk assessment instruments are designed to capture high-risk features in individual cases and are normally accompanied by severity scores (Kelly, Simmons, \& Gregory, 2002). Most of these instruments include either static factors, which change rarely, or dynamic factors, which can change frequently (Kumar \& Simpson, 2005). Because agitation and deterioration are complex and dynamic phenomena, risk assessment instruments should include biological, social, and psychopathological aspects (Amore et al., 2008; Duxbury \& Whittington, 2005), and preferably include situational and environmental factors (National Institute for Clinical Excellence, 2005). Several experts argue that clinical judgement combined with the use of structured risk assessment instruments will result in more efficient clinical decision-making processes (Blok, de Beurs, de Ranitz, \& Rinne, 2010; de Ruiter, 2007; Webster, Hucker, \& Bloom, 2002).

\section{Risk assessment instruments}

We conducted a systematic published work review concerning violence risk assessment instruments to select clinically useful instruments to incorporate in our seclusion reduction program in three psychiatric units in one of the largest university hospitals in the Netherlands and found 19 available instruments (M.D.M Faay, unpubl. data, 2010). The methodological quality of the studies was appraised by using the Quadras standards (Whiting et al., 2004). Based on the reliability, validity, clinical utility, and the methodological quality of the studies, the three following instruments were identified that showed consistently adequate results and could be considered as useful for frequent short-term risk assessment in our units: (i) the Brøset Violence 
Checklist (BVC (Almvik \& Woods, 1999)); (ii) Violence Screening Checklist (VSC (McNiel \& Binder, 1995)) and (iii) Historical Clinical Risk Management-20 (HCR20 (Webster, Douglas, Eaves, \& Hart, 1997)). These three instruments have some disadvantages. The BVC has been studied extensively and is clinically useful but relies dominantly on short-term (within 24 hours) dynamic factors and is most predictive during the first days of hospitalization (Almvik \& Woods, 2003). The VSC mainly captures static factors. The HCR-20 appeared to be less useful in our setting due to the time-consuming clinical interview protocols and the requirement of having access to comprehensive patient records to complete this instrument.

We also identified the Kennedy Axis V (Kennedy, 2003a). This routine outcome measurement instrument was developed to refine the Global Assessment of Functioning (GAF) which is incorporated in Axis V of the DSM-IV (American Psychiatric Association, 1994). The Kennedy Axis V can be used, like the GAF, for the global assessment of patients' functioning, as it captures the clinician's impression and can be used as a treatment outcome measure (Kennedy, 2003b). The instrument captures not only dynamic strength and risk factors in patient functioning, but also important static factors, like previous violence towards self and others and substance misuse (Van de Sande, 2007). Therefore, the Kennedy Axis V can be used for a wider range of patient-related risk factors (Kennedy, 2003b). Because the instrument uses dynamic and static factors, assesses multiple risk factors, and can be used for outcome measurement, the Kennedy Axis V has many benefits compared with contemporary risk assessment instruments.

Only a few studies systematically evaluated the clinimetric properties of the Kennedy Axis V. Bilezikian (1998) found good interrater reliability, varying 0.80-0.91 on different subscales. Ebben (2006) found high interrater reliability of the instrument by comparing scores of psychologists and physicians with an average kappa of 0.85 and concurrent validity of 0.62 as compared to the GAF in a population of psychiatric patients with severe mental illness. However, both studies dealt with small sample sizes and included specific populations, which limits the generalizability of these findings. An Italian study found high interrater reliability between the scores of clinicians rating the Kennedy Axis V, varying 0.63-0.81 as measured with kappa (Mundo, Bonalume, Del Corno, Madeddu, \& Lang, 2010). The Kennedy Axis V is one of the key scales used in a short-term risk assessment model, the crisis monitor (van de Sande et al., 2011), and is in combination with the BVC recommended as the best practice for short-term risk assessment by the Dutch Inspectorate of Health (Inspectorate of Health, 2011). One comprehensive cluster randomized study has been published which focussed on the predictive validity of the crisis monitor and the risk of being exposed to seclusion. This study showed promising results concerning the Kennedy Axis V for short-term risk assessment (van de Sande et al., 2011). The instrument was 
also used in a study aimed to determine risk factors for seclusion and restraint which found the likelihood of being coerced significantly associated with the Kennedy Axis V (Georgieva, Vesselinov, \& Mulder, 2012). Although the Kennedy Axis V is, among other instruments, recommended as best practice in the Netherlands, there are specific aspects that have not been studied before: the use of the instrument in adolescent populations and the utility of the instrument for nursing practice.

According to the Kennedy Axis V manual (Kennedy, 2003b), the instrument is applicable for everyone above the age of 5 years. However, published studies in child and adolescent mental health-care samples are lacking. Risk assessment in adolescents, who are in a vulnerable and dynamic episode of their lives, appears to be rather challenging (Hage et al., 2009). Studies revealed that $37-42 \%$ of the adolescents became violent during hospitalization (Blake \& Hamrin, 2007; Lodewijks, Doreleijers, de Ruiter, \& Borum, 2008). Patients suffering from psychosis are also associated with a higher risk of violent incidents and in some samples there appeared to be a significant correlation between psychosis and violent incidents (Brendel, Wei, \& Edersheim, 2010). Approximately 12-39\% of first-episode psychosis patients are involved in violent incidents (Harris et al., 2010). Therefore, adolescents with mental health problems and adult patients suffering from psychosis need to be monitored frequently by means of structured short-term risk assessment.

The previously described Kennedy Axis $\mathrm{V}$ studies did not evaluate the clinimetric properties of the tool in a sample of nurse raters. In clinical practice, mental health nurses are often the frontline health-care professionals and likely to be exposed to impulsive and dangerous behaviour (Foster et al., 2007; Woods \& Ashley, 2007). Due to their frontline position, they are also likely to recognize the early signs of agitation and violent behaviour to self or others. The quality of this proactive work can be enhanced by a more structured approach (Fluttert et al., 2008). Identifying high agitation levels, poor control over psychiatric symptoms, and temporary lack of adequate social skills are essential for nurses to promote a safe environment (Duxbury, Hahn, Needham, \& Pulsford, 2008). On the other hand, sufficient interrater reliability is crucial for the adequate clinical use of risk assessment tools in day-to-day practice, otherwise consistent risk communication will impede clinical decision-making in unacceptable ways. Therefore, the aim of this study was to determine the interrater reliability and clinical utility of the Kennedy Axis V assessed by mental health nurses in clinical practice at three psychiatric units in a university hospital in the Netherlands. 


\section{Materials and Methods}

\section{Participants}

This cross-sectional study was conducted from February until May 2011 in three psychiatric units in a university hospital in the Netherlands. Unit A and B cater for patients with first-episode psychosis and other psychotic disorders. Unit A is focussed on inpatient care and unit B on outpatients. Unit $\mathrm{C}$ is a locked unit for diagnosis and treatment of adolescents with a variety of mental health problems.

A total of 28 mental health nurses worked on the three units. All licensed and registered nurses working day and evening shifts were included in this study, resulting in a final sample of 23 nurses. This sample included nine nurses from unit A, two from unit B, and 12 from unit C. All 23 nurses were invited to participate in the different data collection methods after they were informed face-to-face and by newsletters about their role in the study protocol. The study protocol was approved by the local medical ethical review committee.

\section{Measures}

\section{Kennedy Axis V}

The Kennedy Axis V is constructed in seven subscales: (i) psychological impairment, including mood changes and psychotic symptoms; (ii) social skills, including interpersonal and communication skills; (iii) violence, including threatening, assaultive, and suicidal behaviour; (iv) activities of daily living and occupational skills, including job or school skills; (v) substance misuse, including alcohol, drugs, and nicotine misuse; (vi) medical impairment, including hypertension and weight gain; and (vii) ancillary impairment, including financial, legal, and housing problems (Kennedy, 2003b). In the Dutch version, an additional scale is used: (viii) motivation for treatment, including the patient's cooperation with clinicians and medication adherence (Mulder, 2000). Each subscale can be scored in the range 0-100. A score above 50 indicates a strength in the patient's functioning on the specific subscale and, subsequently, a score of 50 or lower indicates problematic functioning in a specific area (Kennedy, 2003b). The Kennedy Axis V manual provides brief descriptions of the severity scores to support the completion of the subscales. These descriptions are anchor points and examples of patients' functioning which should generally agree with the clinical impression of the professional (Kennedy, 2003b). For every subscale, a dangerousness score can be calculated which is used to determine the most dangerous rating on the subscales. The lowest score indicates the patient is at risk for adverse outcomes in a specific area 
of functioning. The dangerousness level for each subscale varies 30-50. Those cut-off scores indicate the likelihood of adverse situations (Kennedy, 2003a).

\section{Interrater reliability}

The interrater reliability of the Kennedy Axis V was calculated by comparing the agreement in scores of two nurses for the same patients in similar timeframes. Both daily and weekly scores were used for the calculation of the interrater reliability. The researcher selected patients randomly using a random number generator on the Internet and monitored if the nurses scored these patients independently of each other.

To ensure sufficient power, we calculated that for an estimated population correlation of 0.80 , based on the studies conducted by Ebben (2006), Bilezikian (1998), and Mundo et al. (2010), a two-tailed alpha of 0.05 and power of 0.80 , the instrument should be scored 10 times to calculate Pearson's $r$ (Polit \& Lake, 2010) and 51 times to calculate the intraclass correlation coefficient (ICC) (Bonett, 2002). This is consistent with Terwee et al. (2007) who state that for determining interrater reliability, there should be at least 50 screenings conducted twice.

\section{Clinical utility}

The clinical utility of the Kennedy Axis V was measured using a questionnaire, which was originally developed to investigate the clinical utility of depression screening instruments for patients with stroke using the criteria of Harris and Warren (1995). Clinical utility reflects aspects such as cost of procuring, reproducing, and retrieving information, and issues on how to score and interpret the data as well as the time needed to administer the instrument (Harris \& Warren, 1995). The clinical utility questionnaire was constructed using the two step method of Lynn (1986). The content validity was determined in a five round Delphi survey by an expert panel of four clinical nursing researchers. The clarity of the terminology in relation to clinical practice was further assessed by four expert neuroscience nurses. For this study, the questionnaire was adjusted by eliminating questions focusing on specific clinical aspects of stroke patients. Five expert psychiatric nurses evaluated the content, formulation and layout of the questionnaire for its use in psychiatric settings. Only minor changes were made. The questionnaire includes 10 items focusing on nurses' opinions of the questions stated. The answers are provided on a dichotomous scale ("yes" and "no") with open spaces where the nurses could explain their experiences. Two questions consider the time needed to fill in the questionnaire. Because of the diversity of the questions, no total score is calculated for the questionnaire. 


\section{Procedure}

The Kennedy Axis V was introduced on the units in January 2011 using five different strategies:

1. A plenary lecture on the research protocol and procedures in the team meetings. Also, the Kennedy Axis V manual was provided to all participating professionals.

2. A plenary and interactive lecture considering the basic principles of the instrument and a demonstration of the instrument by means of case vignettes. This training was provided twice on unit $\mathrm{A}$ and $\mathrm{B}$, once on unit $\mathrm{C}$, and strongly recommended for all professionals. Those who were not present were individually trained by the researcher (unit A and B). Because of organizational problems, the nurses at the adolescent unit $C$ that were unable to attend this plenary lecture were instructed by other colleagues.

3. Training on the job while nurses scored patients by means of the Kennedy Axis V.

4. Frequent consensus meetings to address scoring problems in specific cases.

5. A newsletter was published every second week, which included various aspects of working with the Kennedy Axis $\mathrm{V}$ and the proceeding of the study

The first four subscales of the Kennedy Axis V were scored every day, whereas all eight subscales were scored every week for each patient. All scores were noted in the patient's file and were accompanied by short descriptive reports explaining the scores. The dangerousness cut-off scores mentioned in the Kennedy Axis V manual (Kennedy, 2003 b) were not formally used. The ratings were incorporated in the daily reports and multidisciplinary meetings. The principal researcher monitored the instrument fidelity on the units and provided coaching on the job during the research period.

Nurses received the clinical utility questionnaire by an email after using the instrument for 1 month (unit C) or 3 months (unit A and B). The data on interrater reliability were collected throughout the study period.

\section{Data analysis}

The interrater reliability was calculated using the ICC and the Pearson's $r$. The two-way random ICC model was used because the nurses and patients were both considered to be random (Shrout \& Fleiss, 1979). Although Pearson's $r$ is considered unsuitable for calculating interrater reliability (Bland \& Altman, 1986; Fleenor, Fleenor, \& Grossnickle, 1996), we calculated this coefficient to compare our findings with previous studies. An ICC score of 0.49-0.59 indicates moderate agreement, a score of 0.60-0.79 substantial agreement, and a score of 0.80 or higher indicates a high level 
of agreement (Landis \& Koch, 1977). Pearson's $r$ should be 0.80 or higher when used for interrater reliability (Polit \& Beck, 2008). The demographic data and the clinical utility questionnaire data were analysed with descriptive statistics. SPSS version 16.0 for Windows (SPSS Inc, 2007) was used for all data analysis.

\section{Additional data collection and analysis}

The nurses at the adolescent unit $\mathrm{C}$ experienced considerable rating problems and therefore it was decided to stop using the instrument after 1 month. To explore the impeding factors in the rating process in this adolescent setting, additional data were collected. Two groups of three nurses were formed and each group participated in a focus group interview. These interviews were based on the qualitative research principles of Morse and Field (1995) and the focus was twofold:

1. What difficulties did you experience?

2. What are the positive factors you experienced while using the instrument?

The issues the nurses described during the interviews were further explored by indepth questions, clarifying underlying arguments, consequences, and relationships between issues. In the final stage of the interviews, the issues were clustered into categories.

The data were analysed using deductive content analysis as described by Elo and Kyngas (2008). The interviews were transcribed and coded with the categories from the interviews. The categories and accompanying issues from both interviews were combined and presented to the respondents to allow a member check. The issues were clustered by two researchers. Finally, the most frequently named positive and negative issues for each category were presented in a figure.

\section{Results}

\section{Sample characteristics of nurses and patients}

The sample consisted of 23 nurses who participated in different parts of this study. The mean age was 31.3 years and 15 nurses (65.2\%) were female (Table 1). The mean age of the patients was 24.6 years at unit A, 24.7 years at unit B, and 16.3 years at unit C (Table 2). Twenty-five patients on unit A (67.5\%) and seven patients (58.4\%) at unit B suffered from psychosis. Nine patients at unit C (90.0\%) suffered from multiple psychiatric disorders. 
Table 1: Nurses characteristics

\begin{tabular}{|c|c|}
\hline & $\begin{array}{c}\text { Total }(n=23) \\
N(\%)\end{array}$ \\
\hline \multicolumn{2}{|l|}{ No. of nurses } \\
\hline Unit $A$ & $9(39.1)$ \\
\hline Unit B & $2(8.7)$ \\
\hline Unit C & $12(52.2)$ \\
\hline \multicolumn{2}{|l|}{ Age, years } \\
\hline Mean (SD) & $31.3(7.8)$ \\
\hline Range & $23-50$ \\
\hline \multicolumn{2}{|l|}{ Sex } \\
\hline Male & $8(34.8)$ \\
\hline Female & $15(65.2)$ \\
\hline \multicolumn{2}{|l|}{ Education } \\
\hline Master's degree & $3(13.0)$ \\
\hline Registered nurses (Bachelor degree) & $16(69.6)$ \\
\hline Licensed practical nurses & $4(17.4)$ \\
\hline \multicolumn{2}{|l|}{ Work experience in psychiatry, years } \\
\hline Mean (SD) & $7.6(6.4)$ \\
\hline Range & $1-25$ \\
\hline
\end{tabular}

SD, standard deviation.

\section{Interrater reliability}

Within the entire sample of 23 nurses, 17 nurses $(73.9 \%)$ participated in scoring patients in order to measure the interrater reliability. Six nurses were unable to participate because of absence related to illness or holiday leave. In some cases, not all subscales were scored by both nurses for each patient. Table 3 reveals that there were missing scores because the first four subscales should have been scored 78 times and the subscales $5-8$ should have been scored 65 times. Those missing scores were excluded from analysis.

The interrater reliability for the total instrument was 0.79 as measured with both ICC and Pearson (Table 3). The ICC and Pearson values were similar on all subscales. All correlation coefficients of the subscales were more than 0.60 , except for the subscales social skills (0.56) and ancillary impairment (0.06). The mean difference between the scores of two nurses for the same patients differs among units; especially on these subscales, there are outliers in the data from unit $\mathrm{C}$ (Figure 1). Therefore, we repeated the analysis, leaving out the data from unit C. For the subscale social skills, this resulted in an ICC of 0.64 and Pearson 0.65. Both correlation coefficients for the subscale ancillary impairment are 0.18 . 
Table 2: Patients' characteristics

\begin{tabular}{|c|c|c|c|c|}
\hline & $\begin{array}{l}\text { Unit A } \\
(n=37)\end{array}$ & $\begin{array}{c}\text { Unit B } \\
(n=12)\end{array}$ & $\begin{array}{c}\text { Unit C } \\
(n=10)\end{array}$ & $\begin{array}{c}\text { Total } \\
(n=59)\end{array}$ \\
\hline & $\mathrm{N}(\%)$ & $\mathrm{N}(\%)$ & $\mathrm{N}(\%)$ & $\mathrm{N}(\%)$ \\
\hline \multicolumn{5}{|l|}{ Age, years } \\
\hline Mean (SD) & $24.6(8.0)$ & $24.7(5.8)$ & $16.3(2.2)$ & $23.2(7.5)$ \\
\hline Range & $16-55$ & $16-36$ & $12-20$ & $12-55$ \\
\hline \multicolumn{5}{|l|}{ Sex } \\
\hline Male & $30(81.1)$ & $8(66.7)$ & $5(50.0)$ & 43 (72.9) \\
\hline Female & $7(18.9)$ & $4(33.3)$ & $5(50.0)$ & $16(27.1)$ \\
\hline \multicolumn{5}{|l|}{ Diagnosis ${ }^{\dagger}$} \\
\hline Psychotic disorder & $25(67.5)$ & $7(58.4)$ & $1(10.0)$ & $33(55.8)$ \\
\hline Mood disorder & $8(21.6)$ & $5(41.7)$ & $3(30.0)$ & $16(27.1)$ \\
\hline Autism spectrum disorder & $4(10.8)$ & $2(16.7)$ & $5(50.0)$ & $11(18.6)$ \\
\hline Other disorders & $1(2.7)$ & $0(0.0)$ & $4(40.0)$ & $5(8.4)$ \\
\hline Multiple diagnosis & $4(10.8)$ & $2(16.7)$ & $9(90.0)$ & $15(25.4)$ \\
\hline Substance abuse & $21(56.8)$ & $6(50.0)$ & $1(10.1)$ & $28(47.5)$ \\
\hline Ethnicity: Dutch & $30(81.1)$ & $10(83.3)$ & $9(100.0)$ & $49(83.0)$ \\
\hline Involuntary commitment & $11(29.7)$ & $0(0.0)$ & $4(40.0)$ & $15(25.4)$ \\
\hline
\end{tabular}

+More than one diagnosis possible. SD, standard deviation.

Table 3: Interrater reliability of the Kennedy Axis $\mathbf{V}$ used by nurses

Interrater reliability

\begin{tabular}{|c|c|c|}
\hline & ICC & Pearson \\
\hline 1. Psychological impairment $(n=78)$ & 0.78 & $0.78 * *$ \\
\hline 2. Social skills $(n=78) \dagger$ & 0.56 & $0.56 * *$ \\
\hline 3. Violence $(n=77)$ & 0.69 & $0.69 * *$ \\
\hline 4. ADL-occupational skills $(n=76)$ & 0.71 & $0.71^{* *}$ \\
\hline 5. Substance abuse $(\mathrm{n}=62)$ & 0.79 & $0.79 * *$ \\
\hline 6. Medical impairment $(n=62)$ & 0.79 & $0.79 * *$ \\
\hline 7. Ancillary impairment $(n=56) \ddagger$ & 0.06 & 0.06 \\
\hline 8. Motivation for treatment $(n=64)$ & 0.78 & $0.79 * *$ \\
\hline Total $(n=553) \S$ & 0.79 & $0.79 * *$ \\
\hline
\end{tabular}


Figure 1: Mean differences in nurses' ratings on the subscales among units

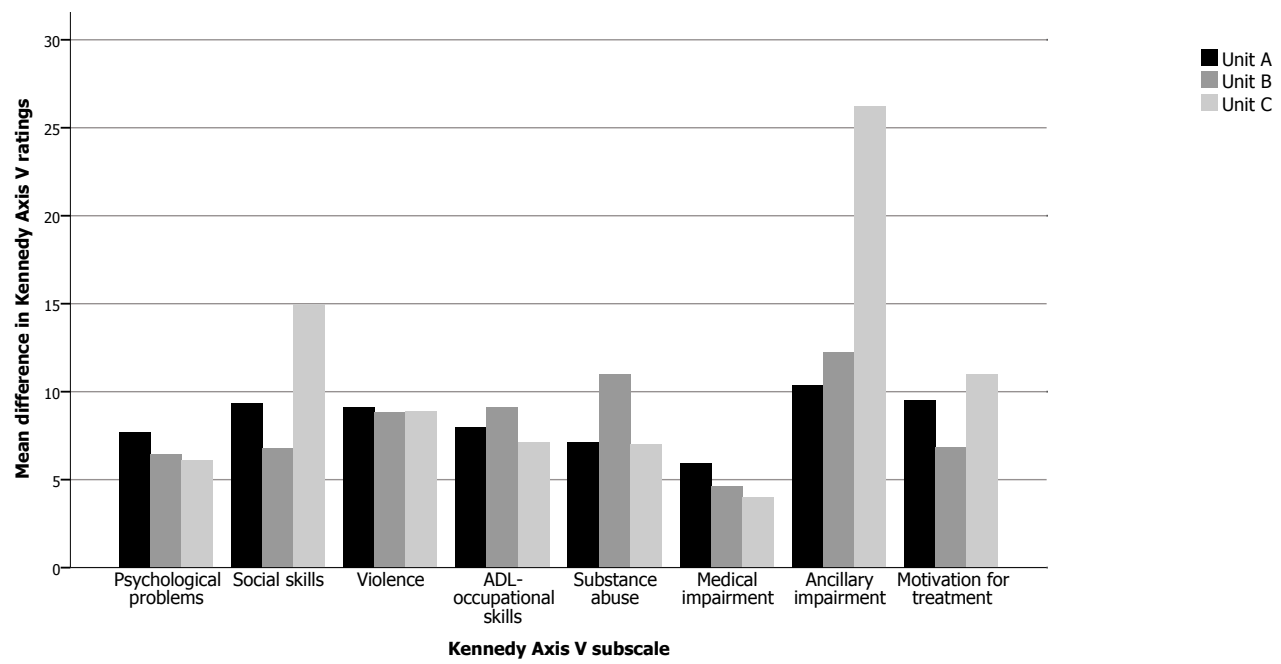

\section{Clinical utility}

From the total sample of 23 nurses, 20 nurses (87\%) completed the clinical utility questionnaire (Table 4). All 20 nurses (100\%) believed that patients could benefit if the staff would frequently use risk assessment instruments. The mean time of scoring the eight subscales was $15.2 \mathrm{~min}$ and, when nurses had scored four times or more, it took $11.6 \mathrm{~min}$ to score the instrument and to write a short report. Fourteen nurses $(73.7 \%)$ considered this time needed to score the instrument unacceptable because they had other priorities and tasks and experienced little profit from using the instrument, as they explained in the questionnaire. Eighteen nurses (90.0\%) had trouble scoring one or more subscales because they found the content description of the subscales not clear enough. On unit $\mathrm{C}$, the nurses could not link the content of the manual to the adolescent population. Thirteen nurses $(72.2 \%)$ could not relate the scores with accompanying interventions to reduce the risk factors. Fourteen nurses $(70.0 \%)$ found that the instrument provided useful information for the (nursing) care plan. Thirteen nurses $(72.2 \%)$ considered the instrument useful within the nursing written report and 12 nurses (70.6\%) considered the instrument to be useful for the weekly multidisciplinary meetings. 
Table 4: Results of the clinical utility questionnaire of the Kennedy Axis V used by nurses

Items on the clinical utility questionnaire Total $(n=20)$

Mean (SD) $\mathrm{N}(\%)$

1. Do you think patients benefit from the use of a risk assessment instrument?

$\begin{array}{ll}\text { Yes } & 20(100) \\ \text { No } & 0(0.0)\end{array}$

2. Is the instrument is easy to score without extra activities?

Yes

No

$12(60)$

3. How much time does it take to score subscales $1-4$ ?

Time in $\min$

Time when scored $<10$ times $(n=9)$

Time when scored $\geq 10$ times $(n=10)$

4. How much time does it take to score subscales $1-8$ ?

$$
\begin{array}{rc}
\text { Time in min } & 5.2(9.2) \\
\text { Time when scored }<4 \text { times }(n=8) & 17.9(11.2) \\
\text { When scored } \geq 4 \text { times }(n=6) & 11.6(4.2)
\end{array}
$$

5. Do you consider the time needed to score to be acceptable?

Yes

No

$14(73.7)$

6. Do you think the subscales are clear?

Yes

$10(50.0)$

No

$10(50.0)$

7. Are the subscales easy to score?

Yes:

No:

$18(90.0)$

7a. Which subscales do you have trouble scoring:

(i) Psychological impairment

(ii) Social skills

$5(27.8)$

(iii) Violence

$12(66.7)$

(iv) $\mathrm{ADL}$-occupational skills

5 (27.8)

(v) Substance abuse

5 (27.8)

(vi) Medical impairment

$2(11.1)$

(vii) Ancillary impairment

8 (44.4)

(vii) Motivation for treatment

$3(16.7)$

8. Is the instrument easily scored? 
9. Do you know which interventions to use according to the score?

$\begin{array}{lc}\text { Yes } & 5(27.8) \\ \text { No } & 13(72.2)\end{array}$

10. Is the instrument easy to use (layout, scores)?

$\begin{array}{ll}\text { Yes } & 14(70) \\ \text { No } & 6(30)\end{array}$

11. Does the instrument provide information that is clinically useful for the (nursing) care plan?

$\begin{array}{ll}\text { Yes } & 14(70.0) \\ \text { No } & 6(30.0)\end{array}$

12. Do you think the instrument is useful within:

Nursing verbal report

Nursing written report

Daily medical verbal report

Weekly multidisciplinary meeting

$12(70.6)$

$A D L$, activities of daily living; $S D$, standard deviation.

\section{Focus group interviews on the adolescent unit}

A sample of 12 nurses from the adolescent unit participated in this study, of whom six nurses $(50 \%)$ participated in the focus group interviews. Four thematic categories emerged from the interviews (Figure 2). The nurses had difficulties in distinguishing the psychiatric impression from developmental and childhood issues. The nurses found that they were insufficiently trained because formal training was provided only once on unit $\mathrm{C}$ and they needed more training on the job. Moreover, they experienced that the Kennedy Axis V manual did not provide enough information for them on how to rate the scale in an age-specific way. The nurses also reported positive experiences. Some described the anchor points in the instrument to observe violent behaviour and found the instrument efficient to use.

Figure 2: Results of the focus group interviews on the adolescent unit C

\begin{tabular}{|c|l|l|l|}
\hline \begin{tabular}{|l|l|} 
Assessment of \\
patient functioning \\
in a more objective \\
way
\end{tabular} & $\begin{array}{l}\text { Patients' current risks, } \\
\text { strengths and skills } \\
\text { needs to be re-assessed } \\
\text { frequently }\end{array}$ & $\begin{array}{l}\text { Ratings will be less time- } \\
\text { consuming in a routine } \\
\text { fashion }\end{array}$ & $\begin{array}{l}\text { Did proof to be a } \\
\text { useful aid for standard } \\
\text { observations and risk } \\
\text { assessment }\end{array}$ \\
\hline \begin{tabular}{|l|l|l|} 
Instrument \\
Time consuming \\
instrument in the \\
beginning due to \\
a comprehensive \\
manual
\end{tabular} & $\begin{array}{l}\text { Population } \\
\text { Concerns about } \\
\text { appropriate ratings } \\
\text { in adolescent } \\
\text { psychiatry }\end{array}$ & $\begin{array}{l}\text { Implementation } \\
\text { training on the job } \\
\text { to refine age specific } \\
\text { ratings }\end{array}$ & \begin{tabular}{l} 
Team configuration \\
\hline implementation process \\
due to major organization \\
changes. Lack of expert \\
in daily practice
\end{tabular} \\
\hline
\end{tabular}




\section{Discussion}

This is one of the few studies that investigates the interrater reliability and clinical utility of the Kennedy Axis $\mathrm{V}$ from a mental health nursing perspective in a sample of adolescent patients and adults with psychotic disorders. The overall interrater reliability of the Kennedy Axis V (0.79) was found to be close to outstanding. Among the challenges experienced, the nurses found it difficult to score the instrument and use interventions related to the scores. On unit C, the nurses found it difficult to use the instrument for the adolescent population.

There are some limitations to this study. The study was relatively small because there were only two wards participating and, even though almost all nurses were included in the study, the number of nurses remains low. Also, because the adolescent unit dropped out of the study, there is little data on interrater reliability on this unit and the results, considering that clinical utility data were collected after using the instrument for only 1 month. This limitation may also be discussed as a strength of this study because different types of wards were involved. Although the adolescent unit was not able to finish the study, additional data were collected exploring the nurses' opinion of using the instrument with adolescents. As far as we know, this aspect has not been studied before. The results of this study indicate promising options to generalize the findings to comparable adult psychiatric units. However, the data on clinical utility revealed that there are many unit-specific factors contributing to the clinical utility of an instrument, such as previous education of nurses and the number of staff. We learned that clinical utility is essential for an adequate implementation process and it is therefore recommended that the instrument is evaluated for every specific unit prior to implementation.

The interrater reliability found in this study is comparable with the findings of Mundo et al. (2010), where the subscales social skills and ancillary impairment also have the lowest agreement (respectively, ICC 0.633 and 0.667). However, in the current study the interrater reliability of the subscale ancillary impairment is much lower than these earlier findings and this may possibly be caused by a lack of information about the patient by the nurses or disagreement considering which problems should be rated. Pearson's $r$ from the study of Ebben (2006) and Bilezikian (1998) was higher than in the current study. However, because of the small sample size $(n=24)$ in the study of Ebben (2006) and the inappropriateness of Pearson's $r$ for measuring interrater reliability, these results could overestimate the actual agreement (Bland \& Altman, 1986). In this study, a larger sample size was used and the correlation coefficients were similar, indicating that our findings reflect the actual agreement (Polit \& Lake, 2010). Furthermore, little is clear about how the scoring procedures took place in previous 
studies. The raters were possibly more experienced using the scales but these articles did not mention this.

This study has several implications for the use of the instrument in adult mental health-care facilities. The nurses on the adult units A and B felt that they captured useful clinical information with the Kennedy Axis V but sometimes struggled with scoring and were unsure which complementary interventions they should use related to the assessed level of patient functioning. These challenges during the implementation processes have also been reported in other structured risk assessment studies (Watts et al., 2004; Webster \& Hucker, 2007).Various authors have emphasized that implementation of risk assessment instruments is a time-consuming process which needs careful planning and the use of multifaceted, tailored implementation strategies (Grol \& Grimshaw, 2003; Hoge, 2010). Several more specific strategies are therefore recommended to adequately implement the Kennedy Axis V in adult mental healthcare facilities:

1. Multiple training sessions and workshops which address how to refine the nursing care plan supported by an assessment tool are highly recommended. The need for adequate training is also emphasized in British risk management best practice evaluations (National Institute for Clinical Excellence, 2005). Continuous education in this area is needed as structured risk assessment is often lacking in basic and post-graduation nurse education curricula (McKenna, Poole, Smith, Coverdale, \& Gale, 2003). Moreover, risk assessment is considered especially challenging for junior registered nurses because they have experienced few risk scenarios and are in the process of learning to prioritize their observations (Whitley, Jacobson, \& Gawrys, 1996). This legitimates prolonged and intensive coaching for junior nurses in the course of the career trajectory, but it is also essential to emphasize the mastery of risk assessment scales in the basic and postBachelor education of nurses.

2. Structured and individual de-escalation plans can facilitate the use of interventions for risk management. These plans should incorporate the specific cut-off scores and corresponding preventive interventions. The Kennedy Axis V cut-off scores for dangerousness were not used in this study but these could facilitate the use of tailored interventions when a patient reaches a certain high-risk level cut-off rating. It should be noted that the relation between risk assessment and risk management is difficult with most instruments (Gilbert, Adams, \& Buckingham, 2011). Like most assessment scales, the Kennedy Axis $\mathrm{V}$ manual also exclusively focuses on assessment issues rather than recommending corresponding interventions. However, in this study, the Kennedy Axis V treatment plan handbook (Kennedy, 
2008) was not incorporated into the training because treatment planning was not the aim of this study. When the scores of the Kennedy Axis V are adequate, valid, and reliable, the use of treatment plans incorporating ratings should be considered.

3. Planning which nurses score which patients should prevent missing scores. The missing scores appear to be caused by nurses who may not have been adequately informed about the present mental state of patients or have not been able to observe the patient more specifically when they started rating. This should not be a problem when nurses have specific patients under their supervision or otherwise nurses should inform each other more in detail to enable adequate Kennedy Axis $\mathrm{V}$ scores. This phenomenon also indicates the importance of being aware of what the quality of patient-nurse interaction has been during the shift. This is specifically important with the ancillary impairment subscale because nurses should be informed about the present mental state of patients and their finances, legal, and housing issues, which are required. We assume that the nurses in this study were generally not well informed about these issues which may have caused lower correlation coefficients on this subscale.

4. Select an adequate time frame to score patients on each unit. We learned that on the outpatient unit B, the daily scores change minimally. On the other hand, we identified that the ratings did fluctuate frequently on the acute units and that the ratings can really support short-term decisions.

5. Careful planning of the implementation period, including conducting a pilot test where some patients are rated. We learned that nurses needed some time to adequately use the instrument and therefore it may be necessary to plan more than 3 months for the implementation.

During this study, the Kennedy Axis V was used alongside the BVC (Almvik \& Woods, 1999), which had been implemented on the units prior to this study and was used structurally. Although this was not the scope of this study, we recommend the use of both instruments because of their complementary qualities. For scoring the Kennedy Axis V, information about the background of the patient is required. The BVC can be scored using observations only and is appropriate during the first days of hospitalization (Abderhalden et al., 2004).Van de Sande et al. (2011) has presented further details concerning the combination of these instruments.

The author of the instrument (Kennedy, 2003a) claims that the instrument can be used for all patients including children over 5 years of age. However, we believe that this needs to be studied in a broader sense because the results on the adolescent 
unit $\mathrm{C}$ show that the nurses experienced problems with using the instrument for adolescents. The Kennedy Axis V captures violence (to self and others) in a dynamic way and provides a clinical impression of the level of self-control regarding anger, which is a developmental challenge in the adolescent phase. Adolescents admitted to acute psychiatric units are in the process of developing self-control competencies that they did not have before. Using the findings of the focus group interviews, the rating problems need to be explored more specifically in the future with use of case vignettes and refined training.

There is a need for similar studies on a larger scale. More specifically, all clinimetric properties of the instrument should be structurally evaluated in multicentre longitudinal studies after the instrument is adequately implemented using the recommended strategies. Especially, the predictive validity of the instrument should be evaluated by comparing the dangerousness scores with patients' outcomes, such as violence, seclusion, suicide, self-harm, self-neglect, and recovery, to learn more about the instrument and its use for short-term risk assessments. Because of the problems encountered in the implementation process, the effects of various implementation strategies should be studied to maximize effectiveness and cost-efficiency.

\section{Conclusion}

This study has shown that the Kennedy Axis V has substantial interrater reliability when used by mental health nurses. However, accurate and thorough implementation of the instrument is essential because of the challenges experienced with scoring the instrument. Another common challenge with risk assessment is the adequate use of specific interventions related to scores which was also encountered in this study. This challenge may be addressed by: (i) specific structured and continuous education for nurses; (ii) structured incorporation of the instrument in the treatment planning meetings on the units; and (iii) the use of multidisciplinary risk management plans with preventive interventions. Considering that the low amount of data from the adolescent unit is a limitation of this study, the rating problems on the adolescent units need to be explored more specifically in the future in terms of specific case vignettes and refined training. The Kennedy Axis $\mathrm{V}$ requires a solid implementation strategy and it takes time for nurses to become accustomed to scoring. However, when the instrument is adequately implemented, it may help with objectivity in clinical decision-making. The Kennedy Axis V can be used for short-term risk assessment and thereby contribute to a reduction of severe self-neglect, suicide, self-harm, and violence. 



\title{
CHAPTER 4
}

\section{Aggressive behaviour, hostility, and associated care needs in patients with psychotic disorders: a 6-year follow-up study}

\author{
Margo Faay \\ Jim van Os
}

The Genetic Risk and Outcome of Psychosis (GROUP) Investigators

Frontiers in Psychiatry (2020), 10, 934 
Hostility and aggressive behaviour in patients with psychotic disorders are associated with demographic and clinical risk factors, as well as with childhood adversity and neglect. Care needs are an essential concept in clinical practice; care needs in the domain of safety for others reflect the actual problem the patient has. Hostility, aggressive behaviour and associated care needs, however, are often studied in retrospect.

In a sample of 1119 patients with non-affective psychotic disorders, who were interviewed three times over a period of 6 years, we calculated the incidence of hostility, self-reported maltreatment to others and care needs associated with safety for other people ("safety-to-others"). Regression analysis was used to analyse the association between these outcomes and risk factors. The population attributable fraction (PAF) was used to calculate the proportion of the outcome that could potentially be prevented if previous expressions of adverse behaviour were eliminated.

The yearly incidence of hostility was $2.8 \%$, for safety-to-others $0.8 \%$ and for maltreatment this was $1.8 \%$. Safety-to-others was associated with previous hostility and vice versa, but, assuming causality, only $18 \%$ of the safety-to-others needs was attributable to previous hostility while $26 \%$ was attributable to impulsivity. Hostility, maltreatment and safety-to-others were all associated with number of unmet needs, suicidal ideation and male sex. Hostility and maltreatment, but not safety-to-others, were associated with childhood adversity. Neither safety-to-others, maltreatment nor hostility were associated with premorbid adjustment problems.

The incidence of hostility, self-reported aggressive behaviours and associated care needs is low and linked to childhood adversity. Known risk factors for prevalence also apply to incidence and for care needs associated with safety for other people. Clinical symptoms can index aggressive behaviours years later, providing clinicians with some opportunity for preventing future incidents.

\section{ABSTRACT}




\section{Introduction}

Most patients with psychosis are not violent but there is an increased risk compared with the general public (Volavka, 2008). Aggressive and hostile behaviour in patients with psychosis has a major impact on patients' families, health care workers and society in general, but most of all on patients. Hostile behaviour can decrease the quality of life and has been associated with poorer social functioning (Lahera et al., 2015). Several demographic and clinical risk factors have been reported such as male sex, socioeconomic deprivation, cannabis use, suicidality and clinical symptoms such as impulsiveness and excitement (Hodgins \& Klein, 2017; Large \& Nielssen, 2011; Moulin, Baumann, et al., 2018; Moulin, Golay, et al., 2018; Witt et al., 2014; Witt et al., 2013), although there are some differences between these studies.

In addition to demographic and clinical risk factors, factors in childhood may also increase the risk for adult aggressive and hostile behaviour. Physical and sexual adversity during childhood was significantly associated with later violence in patients with psychotic disorders (Bosqui et al., 2014; Swanson et al., 2006; Witt et al., 2013). Aspects of premorbid social adjustment, such as difficulties in social relationships during the stages of childhood and adolescent development, could also increase the risk of future violent behaviour among patients diagnosed with schizophrenia (Fresán et al., 2004). Insight in early risk factors can contribute not only to identifying patients at risk but also to clarifying the pathways leading to violence, opening the way to treatment (Volavka \& Citrome, 2011).

While most studies use violence or aggressive incidents as outcome to determine risk factors, these incident rates alone may not reflect the actual problem. The concept of care needs should be considered in addition to incident outcomes. Care needs in the domain of safety for others are essential in clinical practice for both patient and clinician. Care needs allow patients to clarify the impact on their daily lives and the need for care they require to cope with these problems. For the clinician, insight in these needs enables risk management in the domain of safety. Little is known about the relation with other indicators for aggressive behaviours and if the established risk factors for violence or aggressive incidents also apply to care needs in the domain of safety for others.

Many studies have identified risk factors and rates for aggressive behaviour in patients with psychotic disorders in analyses that were cross-sectional or in retrospect. Prospective data can be more informative for incidence, causality and to indicate the predictive value of risk factors. These data can thus help in the management of hostile and aggressive behaviours.

In this paper, we report the analyses of violence, hostility and associated care needs of the GROUP study, a naturalistic multicentre, six-year prospective cohort 
study in a sample of patients with non-affective psychotic disorders. The aim was to analyse the incidence, persistence, risk factors and attributable fraction for hostility, self-reported aggressive behaviour and associated care needs in a population of patients with psychosis.

\section{Methods}

\section{Study design}

The GROUP study is described in detail elsewhere (Korver et al.,2012).A representative cohort of patients aged 16 to 50 years, with a diagnosis of a non-affective psychotic disorder according to the DSM-IV (American Psychiatric Association, 2000), was included. The study also included first-degree family members of these patients and healthy controls, but for the current paper only data from patients were used. In selected representative geographical areas in the Netherlands and Belgium, patients were identified through clinicians whose caseload was screened for inclusion criteria. Subsequently, a group of patients presenting consecutively at these services either as outpatients or as inpatients were recruited. At baseline and then after three and six years, a number of questionnaires, tests and clinical interviews were administered.The study protocol was approved by the Ethical Review Board of the University Medical Centre Utrecht and by local review boards of each participating site. All participants gave written informed consent.

\section{Measures}

\section{Outcome measures}

The three main outcome measures were: the hostility item of the Positive and Negative Syndrome Scale (PANSS (Kay et al., 1987)), maltreatment to others (single question) and the safety- to-others item of the Camberwell Assessment of Need Short Appraisal Schedule (CANSAS (Slade, Beck, Bindman, Thornicroft, \& Wright, 1999)).

Hostility is one of the items on the PANSS positive subscale and is defined as "verbal and nonverbal expressions of anger and resentment, including sarcasm, passive aggressive behaviour, verbal abuse and assaultiveness." Thus, hostility can include a relatively mild to moderate expression of violence. All PANSS items are scored on a scale of 1-7. A score of 1 indicates the item is absent, a score of 7 indicates an extreme level of psychopathology. All 30-itmes of the PANSS were administered at the three study visits: baseline, year 3 and year 6 .

Safety-to-others is one of the 22 items on the CANSAS and is used to 
measure care needs associated with safety for other people. The CANSAS allows clinicians and patients together to identify the needs for care and can subsequently assist in care planning. The CANSAS contains the first question of the Camberwell Assessment of Needs (CAN (Phelan et al., 1995)). The safety-to-others item is assessed with the question: "do you think you could be a danger to other people's safety?" and is scored, like all CANSAS items, as "no problem," "need" or "unmet need." A met need indicates a moderate problem but for which the patient receives some sort of intervention. An unmet need indicates a current serious problem for which no intervention has been initiated. The CANSAS was administered at all three visits.

Maltreatment directed at others was assessed with the question: "did you attack or abuse anybody?" At baseline, the question was aimed at lifetime maltreatment with a distinction between maltreatment prior and after psychosis onset. At followup, patients were asked if they attacked or abused anybody in the past three years (i.e. between the study visits).

\section{Other measures}

Next to these three outcome measures, demographic items and known clinical and early risk factors were incorporated in the analysis.

Demographic items were age (in years), sex $(0=$ male, $1=$ female), education (no/primary, lower secondary, lower vocational, higher secondary, higher vocational, university) and ethnicity $(0=$ white, $1=$ other). Previous studies found both age and lower education risk factors for violent or aggressive behaviour (Large \& Nielssen, 2011; Swanson et al., 2008).

Clinical risk factors included IQ, cannabis use, other PANSS items, number of CANSAS unmet needs and suicidality. Lower intelligence is linked to violent behaviour in the general population and in patients with psychosis (Volavka, 2008), although a large meta-analysis found no relationship (Witt et al., 2013). IQ at baseline and at 3-year follow-up was estimated with use of the 4-subtest version of the Wechsler Adult Intelligence Scale (WAIS-III (Blyler, Gold, Iannone, \& Buchanan, 2000)). At year 6, IQ was estimated with a 15-minute version of the WAIS-III short form (Velthorst et al., 2013). Cannabis use is a well-known risk factor for violent behaviour (Hodgins \& Klein, 2017; Moulin, Baumann, et al., 2018). Cannabis use in the past 12 months (yes/no) was measured with the Composite International Diagnostic Interview (CIDI (World Health Organization (WHO), 1990)) at all three study visits.

As for other PANSS items, we selected items that were previously associated with aggression or incorporated in the same PANSS cluster as hostility: P4 excitement, G4 tension, G8 uncooperativeness and G14 poor impulse control (Lehoux, Gobeil, Lefebvre, Maziade, \& Roy, 2009; Lindenmayer, Bernstein-Hyman, \& Grochowski, 1994; Moulin, Golay, et al., 2018; Witt et al., 2013). Excitement indicates 
"hyperactivity as reflected in accelerated motor behaviour, heightened responsivity to stimuli, hypervigilance or excessive mood lability." Tension indicates "overt physical manifestations of fear, anxiety and agitation." Uncooperativeness is defined as "active refusal to comply with the will of significant others, including the interviewer, hospital staff or family, which may be associated with distrust, defensiveness, stubbornness, negativism, rejection of authority or belligerence." Poor impulse control, sometimes called "impulsivity," is defined as "disordered regulation and control of action on inner urges, resulting in sudden, unmodulated, arbitrary, or misdirected discharge of tension and emotions without concern about consequences." The PANSS total scores for the positive (P1-P7), negative (N1-N7) and general subscale (G1-G16) are presented to describe the current sample. There are suggestions that unmet needs on the CAN are associated with violence (Martinez-Martin et al., 2011). The mean number of care needs was therefore also incorporated as a risk factor. Suicidality is linked to aggressive behaviour in earlier work (Witt et al., 2014). In the current study, suicidality was measured with both the CANSAS, the Community Assessment of Psychic Experiences (CAPE (Konings, Bak, Hanssen, van Os, \& Krabbendam, 2006)) and with a separate question concerning suicide attempts. The suicidality item on the CANSAS hereafter is called safety-to-self ("do you ever have thoughts of harming yourself?") and was scored like other CANSAS items. The CAPE was developed as a self-report scale to measure lifetime psychotic experiences. Suicidality is assessed with the question: "do you ever feel like you do not want to live anymore?" and rated from 0 (never) to 3 (almost always). The CAPE was administered at all three study visits. The clinical interview question exploring suicide attempts was comparable with the maltreatment item: "did you attempt to commit suicide?" Again, at baseline, the question was aimed at lifetime suicide attempts and at follow-up, patients were asked if they attempted to commit suicide in the past three years (i.e. between the study visits).

Early risk factors incorporated in this analysis included premorbid adjustment problems and childhood adversity. The Cannon-Spoor Premorbid Adjustment Scale (PAS (Cannon-Spoor, Potkin, \& Wyatt, 1982)) is aimed at evaluating the level of functioning over several phases of life before the onset of the psychotic illness. A higher PAS score indicates worse premorbid functioning. The PAS was administered at baseline. Childhood adversity events were assessed with the Childhood Trauma Questionnaire 25 item short form (CTQ (Bernstein et al., 1994)), which is rated with a Likert scale from 1 (never true) to 5 (very often true). The total score is the mean of the 25 items. Conform previous analyses with this dataset, the scores were dichotomized differentiating a high and low adversity group with the cut-off at the 80th percentile of scores for the healthy control subjects (van Os, Marsman, van Dam, Simons, \& Investigators, 2017). The CTQ was administered once, either at baseline or at 3-year follow-up. 


\section{Data analysis}

Release 7 of the GROUP database was used for the analyses. All analyses were done using Stata 15.1 (StataCorp, 2017). PANSS hostility scores were dichotomized when used as dependent variable in two categorizations: hostility and high hostility. For hostility, scores of 1 were labelled as "no hostility" and scores $>1$ as evidence for hostility. However, a PANSS score of 2 indicates "questionable pathology" (Kay et al., 1987) and is therefore a low threshold to label as "hostile behaviour." The primary outcome measure for this analysis is high hostility, in line with earlier work (Volavka et al., 2016). For high hostility, scores of 1 and 2 were labelled as no hostility and scores $\geq 3$ were labelled as high hostility. The continuous PANSS hostility score was used for analysis of hostility as an independent variable. For the CANSAS items safety-to-self and safety-to-others, absence or presence of a need was used, without differentiation between met and unmet needs. Thus, the CANSAS need outcomes were dichotomized as need (met or unmet need) or no need. After preparation of these items, four analyses were performed in order to obtain: 1) incidence rates 2) persistence rates, 3) risk factors and 4) the attributable fraction.

First, we calculated the incidence. Data was set for survival analysis using the stset routine in Stata (StataCorp, 2017). Failure was defined as a score on CANSAS safety-to-others, maltreatment, hostility or high hostility, occurring for the first time after baseline. Failures already existing at baseline (for example, patients with PANSS hostility $=2$ at baseline) were therefore excluded from this analysis. Incidence was calculated using failures in single-failure per subject data, meaning only the first occurrence of one of the outcome measures was used to calculate the incidence. The maltreatment item was defined cumulatively including incidents occurring during lifetime (baseline) or between the study visits (year 3 and 6). Hostility was reported over the past week and CANSAS safety-to-others over the past month. In calculating their incidences, we thus assumed that the hostility and CANSAS safety-to-others scores were representative for the behaviour over the past three years.

Second, persistence was measured. Persistence stands for continuation of the item at the next visit. For example, patients with a PANSS hostility score both at year 3 and at year 6. Lagged data were used, indexing the status of the outcome in question at the previous visit. The persistence rates thus indicate the number and proportion of patients that scored on the item at two subsequent visits.

Third, the associations between concurrent risk factors (measured at the same time point) and the four main incidence outcome measures (CANSAS safetyto-others, hostility, high hostility and maltreatment) were calculated. Associations were expressed as hazard ratios $(H R)$ and $95 \%$ confidence intervals $(C I)$ from Cox proportional hazard regression analysis. The HR describes the relative increase in the rate of the occurrence of the event in one group compared with the other group 
(Sutradhar \& Austin, 2018). Although HR is not the same as Relative Risk, the two measures approach each other when the incidence of the outcome in question is low. All HR were adjusted for age and sex. Other relevant variables were incorporated in the Cox regression model.

Fourth, in order to indicate the clinical relevance in terms of preventive potential, the Population Attributable Fraction (PAF) was calculated. The PAF gives an estimation of the proportion of the outcome that could have been prevented if the lagged predictor, i.e. measured at the previous time point, was completely eliminated. For example, the proportion of maltreatment scores that could have been prevented if hostility at the previous visit had been successfully treated. The PAF was calculated from the logistic regression model of incident outcomes with the Stata PUNAF package (Newson, 2013).

\section{Results}

\section{Sample characteristics}

At baseline, 1119 patients were included. In year 3, 811 patients participated and at year 6, 662 patients participated. Patient characteristics are presented in Table 1. The DSM-IV-TR diagnoses of the patients were as follows: schizophrenia and related disorders (DSM-IV-TR code 295.x; $\mathrm{n}=945$ [84\%]), other psychotic disorders (DSM-IV-TR code 297/298; $\mathrm{n}=149$ [13\%]), and psychotic illness in the context of substance abuse or somatic illness $(n=9[1 \%])$. Six patients had a missing diagnosis but fulfilled inclusion criteria, and 11 patients had a final diagnosis of affective psychosis but fulfilled criteria of clinical diagnosis of nonaffective psychosis at study entry; these individuals were retained in the sample assuming subtle diagnostic changes between the time of identification for inclusion and actual assessment that could occur in any patient included in the cohort at any time.

\section{Incidence rates}

At baseline,127 patients (12.8\%) had a CANSAS safety-to-others need. Across the three visits, a total of 162 patients yielded 185 events of CANSAS safety-to-others. From baseline, 32 patients had a new CANSAS safety-to-others in single failure per subject data (Table 2). The yearly incidence thus was $0.8 \%$. Kaplan-Meier survival estimates for CANSAS safety-to-others, differentiated by patients with and without PANSS hostility, maltreatment, CANSAS safety-to-self and childhood trauma are shown in Figure 1. 
At baseline, 188 patients $(18.0 \%)$ had a hostility score $>1$. A total of 300 patients yielded 367 hostility events across the three visits. After baseline, 104 patients had a hostility event in single failure per subject data, yielding a yearly incidence of $2.8 \%$. For high hostility, 99 patients (9.5\%) presented this outcome at baseline. A total of 161 patients yielded 184 high hostility events. After baseline, 57 patients had a new high hostility event in single failure per subject data, thus a yearly incidence of $1.4 \%$.

At baseline, 188 (20\%) patients had a history of maltreatment of others. Of these 188 patients, 53 (5.6\%) had these problems prior to psychosis onset, while 135 patients $(14.4 \%)$ indicated the maltreatment took place after psychosis onset. A total of 255 patients collectively had 288 maltreatment scores. After baseline, 66 patients had a maltreatment score in single failure per subject data, leading to a yearly incidence of $1.8 \%$. The yearly incidence of maltreatment in patients with a CANSAS safety-toothers was $9.7 \%$ versus $1.5 \%$ in patients without a CANSAS safety-to-others.

Figure 1: Kaplan-Meier survival estimates for CANSAS safety-to-others
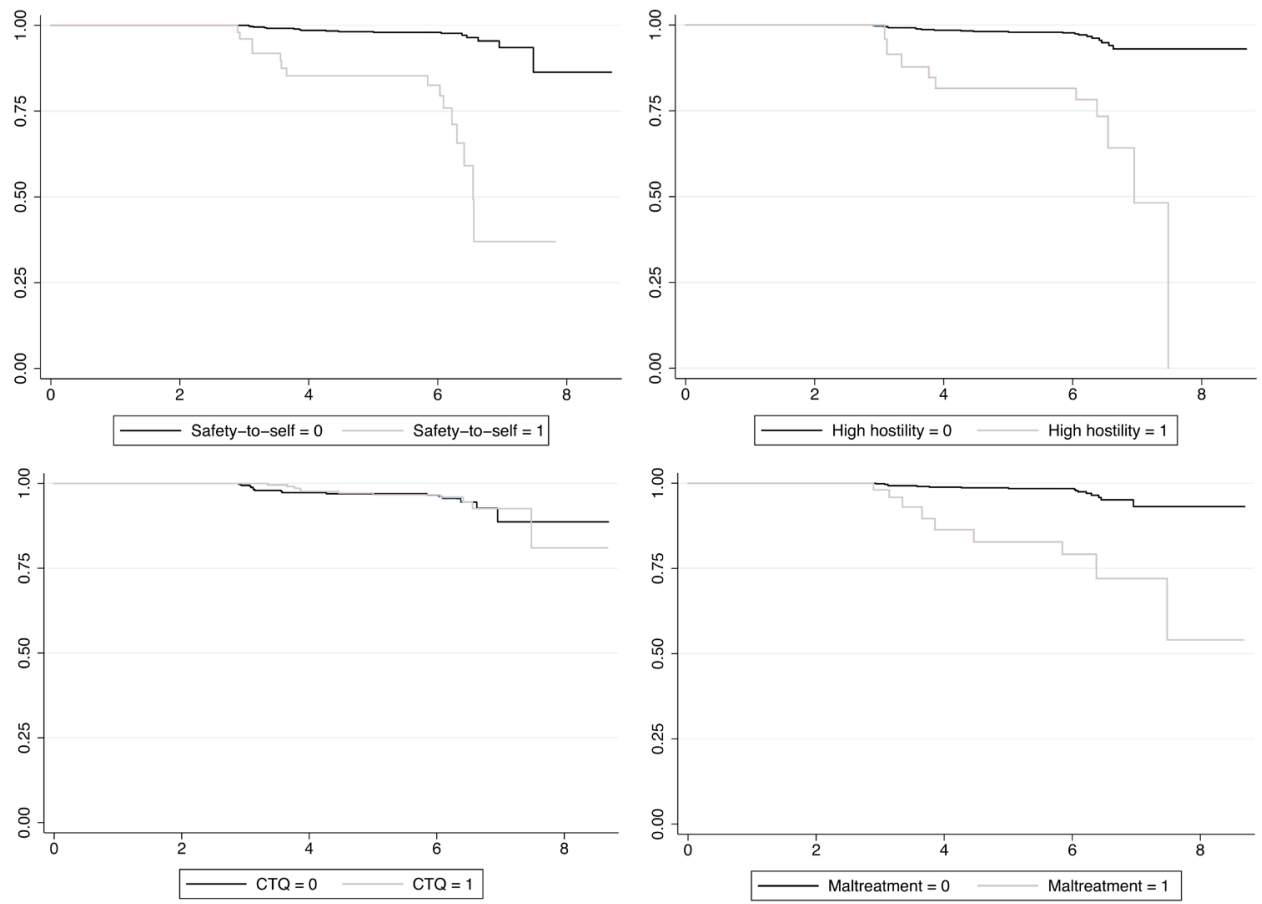

Survival estimates for CANSAS safety-to-others, differentiated by patients with and without high hostility, childhood trauma, maltreatment and CANSAS safety-to-self met or unmet need; X-axis is analysis time in years; CANSAS safety-to-others and safety-to-self item of the Camberwell Assessment of Need Short Appraisal Schedule; hostility item of the Positive and Negative Syndrome Scale with scores >2; CTQ, Childhood Trauma Questionnaire dichotomized with the cut-off at the 80th percentile of scores for the healthy control subjects. 
Table 1: Demographics

\begin{tabular}{|c|c|c|c|}
\hline & $\begin{array}{l}\text { Baseline } \\
\mathrm{n}=1119 \\
\mathrm{~N}(\%)\end{array}$ & $\begin{array}{l}\text { Year } 3 \\
n=811 \\
N(\%)\end{array}$ & $\begin{array}{l}\text { Year } 6 \\
n=662 \\
N(\%)\end{array}$ \\
\hline Mean (SD) & $27.60(7.98)$ & $30.60(7.22)$ & $33.62(7.27)$ \\
\hline $\begin{array}{r}\text { Male } \\
\text { Female }\end{array}$ & $\begin{array}{l}852(76.14) \\
267(23.86)\end{array}$ & $\begin{array}{l}623(76.82) \\
188(23.18)\end{array}$ & $\begin{array}{l}504(76.13) \\
158(23.87)\end{array}$ \\
\hline $\begin{array}{l}\text { White } \\
\text { Other }\end{array}$ & $\begin{array}{l}859(76.76) \\
260(23.24)\end{array}$ & $\begin{array}{l}664(81.87) \\
147(18.13)\end{array}$ & $\begin{array}{l}551(83.23) \\
111(16.77)\end{array}$ \\
\hline $\begin{array}{r}\text { No/primary } \\
\text { Lower secondary } \\
\text { Lower vocational } \\
\text { Higher secondary } \\
\text { Higher vocational } \\
\text { University } \\
\text { Unknown }\end{array}$ & $\begin{array}{c}151(13.49) \\
341(30.47) \\
183(16.35) \\
270(24.13) \\
98(8.76) \\
43(3.84) \\
33(2.95)\end{array}$ & $\begin{array}{c}59(7.27) \\
225(27.74) \\
187(23.06) \\
196(24.17) \\
89(10.97) \\
54(6.66) \\
1(0.12)\end{array}$ & $\begin{array}{c}32(4.83) \\
150(22.66) \\
182(27.49) \\
148(22.36) \\
91(13.75) \\
58(8.76) \\
1(0.15)\end{array}$ \\
\hline Employment & $\begin{array}{l}513(51.25) \\
488(48.75)\end{array}$ & $\begin{array}{l}395(55.79) \\
313(44.21)\end{array}$ & $\begin{array}{l}359(64.45) \\
198(35.55)\end{array}$ \\
\hline Age of onset psychosis & $22.98(7.92)$ & $22.46(6.76)$ & $22.39(6.72)$ \\
\hline Duration of illness in years & $4.98(4.46)$ & $8.46(4.44)$ & $11.57(4.54)$ \\
\hline Hospitalization & $\begin{array}{l}792(78.88) \\
212(21.12)\end{array}$ & $\begin{array}{l}294(40.11) \\
439(59.89)\end{array}$ & $\begin{array}{l}183(30.76) \\
412(69.24)\end{array}$ \\
\hline Mean (SD) & $1.98(0.88)$ & $1.96(.89)$ & $1.94(.88)$ \\
\hline CTQ $80^{\text {th }}$ percentile & $\begin{array}{l}335(44.37) \\
420(55.63)\end{array}$ & $\begin{array}{l}300(44.05) \\
381(55.95)\end{array}$ & $\begin{array}{l}225(40.98) \\
324(59.02)\end{array}$ \\
\hline Mean (SD) & $96.08(15.33)$ & $99.19(16.32)$ & $101.69(17.38)$ \\
\hline $\begin{array}{r}\text { Cannabis use in past } 12 \text { months } \\
\text { Yes } \\
\text { No }\end{array}$ & $\begin{array}{l}417(38.12) \\
677(61.88)\end{array}$ & $\begin{array}{l}197(24.97) \\
592(75.03)\end{array}$ & $\begin{array}{l}142(22.15) \\
499(77.85)\end{array}$ \\
\hline $\begin{array}{r}\text { Never } \\
\text { Sometimes } \\
\text { Often } \\
\text { Almost always }\end{array}$ & $\begin{array}{c}387(44.18) \\
366(41.78) \\
93(10.62) \\
30(3.42)\end{array}$ & $\begin{array}{l}405(54.51) \\
246(33.11) \\
68(9.15) \\
24(3.23)\end{array}$ & $\begin{array}{l}339(56.69) \\
203(33.95) \\
36(6.02) \\
20(3.34)\end{array}$ \\
\hline Suicide attempts & $\begin{array}{l}228(22.51) \\
785(77.49)\end{array}$ & $\begin{array}{l}89(12.23) \\
639(87.77)\end{array}$ & $\begin{array}{c}53(8.88) \\
544(91.12)\end{array}$ \\
\hline $\begin{array}{l}\text { CANSAS number of unmet needs } \\
\text { Mean (SD) }\end{array}$ & $3.24(2.90)$ & $2.26(2.36)$ & $2.41(2.40)$ \\
\hline
\end{tabular}


CANSAS Safety-to-self

$$
\begin{array}{rr}
\text { No problem } & 844(84.91) \\
\text { Met or unmet need } & 150(15.09)
\end{array}
$$

$$
\begin{gathered}
719(93.13) \\
53(6.87) \\
740(96.10) \\
30(3.90)
\end{gathered}
$$

$577(92.77)$

$45(7.23)$

CANSAS Safety-to-others

$$
\text { No problem }
$$

$862(87.16)$

$594(95.50)$

Met or unmet need

$127(12.84)$

$28(4.50)$

Maltreatment to others

$$
\text { Yes } 188(20.04)
$$

$64(9.48)$

$611(90.52)$

$36(6.34)$

$532(93.66)$

PANSS total

$$
\text { Mean (SD) } \quad 54.41(16.80)
$$

$46.79(14.24)$

$47.22(15.86)$

PANSS positive items $\mathrm{P} 1-\mathrm{P} 7$

Mean (SD) $\quad 12.70(5.33) \quad 11.00(4.52) \quad 11.61(5.37)$

PANSS negative items N1-N7

$$
\text { Mean (SD) } \quad 13.94(5.95) \quad 11.75(5.10) \quad 11.73(5.18)
$$

PANSS general items G1-G16

Mean (SD) $27.90(8.40)$

$24.05(7.18)$

$23.92(7.59)$

PANSS P7 Hostility (dichotomous)

$\begin{array}{rccc}\text { No }(1) & 856(81.99) & 692(88.95) & 535(85.19) \\ \text { Yes }(\geq 2) & 188(18.01) & 86(11.05) & 93(14.81)\end{array}$

PANSS P7 high Hostility

(dichotomous)

$\begin{array}{cccc}\text { No }(\leq 2) & 945(90.52) & 736(94.60) & 585(93.15) \\ \text { Yes }(\geq 3) & 99(9.48) & 42(5.40) & 43(6.85)\end{array}$

PANSS P7 Hostility (continuous)

$\begin{array}{llll}\text { Mean (SD) } & 1.31(.77) & 1.17(.53) & 1.26(.78)\end{array}$

PANSS P4 Excitement

$\begin{array}{llll}\text { Mean (SD) } & 1.34(.77) & 1.26(.67) & 1.28(.69)\end{array}$

PANSS G4 Tension

$$
\begin{array}{llll}
\text { Mean (SD) } & 1.87(1.04) & 1.74(.97) & 1.67(.95)
\end{array}
$$

PANSS G8 Uncooperativeness

Mean (SD) $\quad 1.23(.64) \quad 1.14(.54) \quad 1.15(.61)$

PANSS G14 Poor impulse control

Mean (SD)

$1.32(.76)$

$1.18(.58)$

$1.20(.60)$

PAS, Premorbid Adjustment Scale; CTQ, Childhood Trauma Questionnaire dichotomised with the cutoff at the $80^{\text {th }}$ percentile of scores for the healthy control subjects; CAPE, community assessment of psychic experiences; CANSAS, Camberwell Assessment of Need Short Appraisal Schedule; PANSS, Positive and Negative Syndrome Scale, denominators change due to incomplete data. 


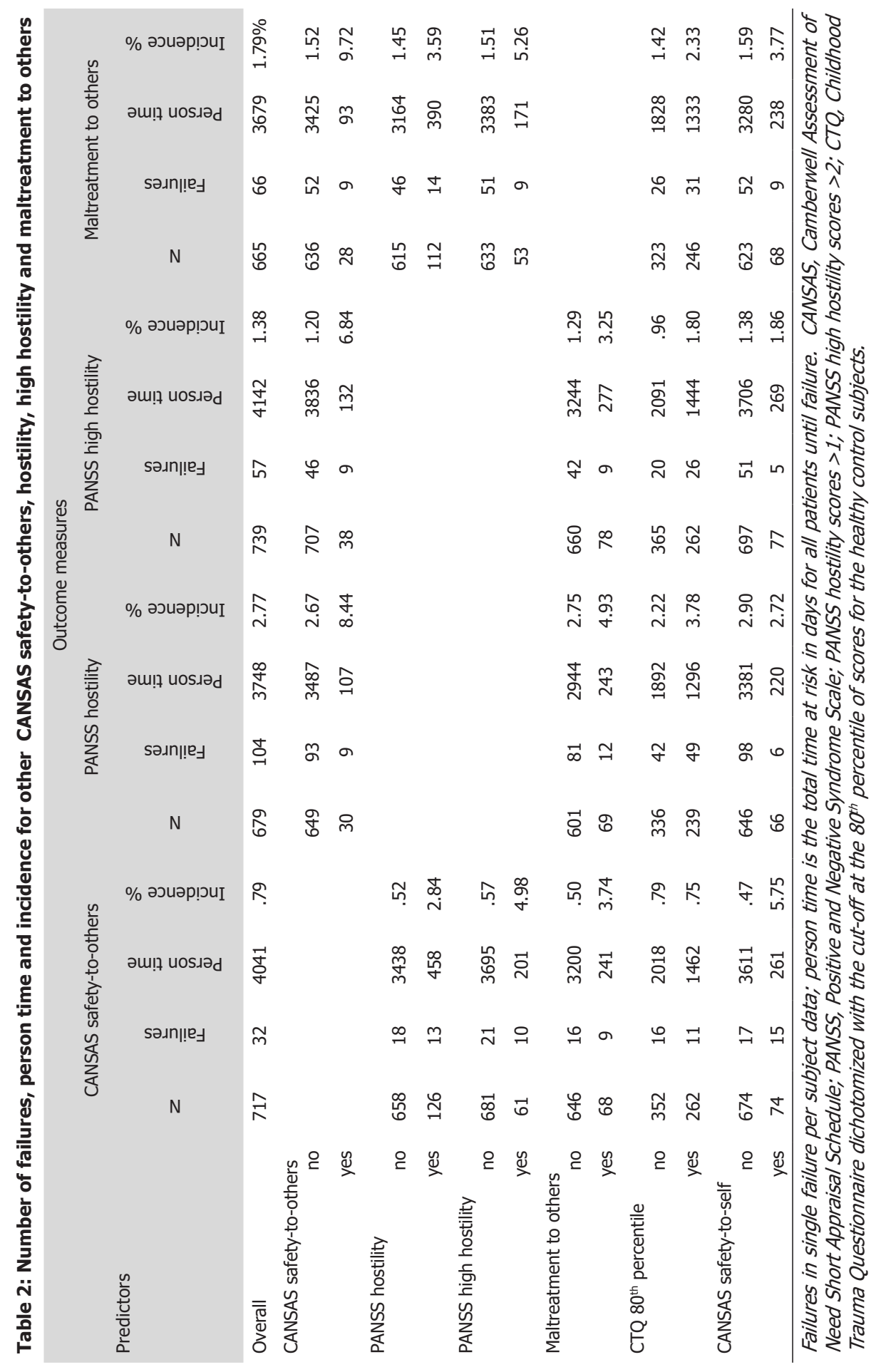




\section{Persistence rates}

Of the 99 CANSAS safety-to-others events at time point t, 17 (17.2\%) had a persistent CANSAS safety-to-others again at the next visit $t+1$. For hostility, the persistence rate was $27.4 \%$ (46 patients) and for high hostility this was 16.1\% (13 patients). For maltreatment, 27 patients (17.5\%) had a maltreatment score again at the next visit.

\section{Risk factors}

Maltreatment was significantly associated with CANSAS safety-to-others (HR 6.28 $p=.00$; Table 3$)$ and vice versa (HR $4.50 p=.00)$, corrected for CANSAS safety-toself. CANSAS safety-to-others (HR $3.58 p=.00)$ and maltreatment $(1.88 p=.04)$ were significantly associated with hostility. In the Cox regression model for CANSAS safety-to-others, there was an association with the PANSS items poor impulse control (HR $2.38 p=.00)$, hostility (HR $1.56 p=.03)$ and uncooperativeness (HR .53 $p=.02)$ but not with excitement (HR $1.11 p=.64)$ or tension (HR $1.11 p=.56)$.

CANSAS safety-to-others (HR 1.86; $p=.00)$, hostility $(H R 1.36 ; p=.01)$ and maltreatment (HR $1.82 p=.00)$ were associated with CAPE suicidality. However, only CANSAS safety-to-others was associated with CANSAS safety-to-self (HR 15.32; $p=.00)$. Hostility (HR $1.65 p=.02)$ and maltreatment (HR $1.74 p=.04)$ were associated with childhood adversity but CANSAS safety-to-others was not (HR .98 $p=.96)$. Neither hostility, maltreatment nor CANSAS safety-to-others were associated with premorbid adjustment.

\section{Population attributable fraction}

The PAF for hostility in predicting CANSAS safety-to-others was 18\% (95\% CI-.00 to .34), indicating that, assuming causality, $18 \%$ of these safety-to-others needs could have been prevented if hostility at the previous visit was eliminated. For high hostility, the PAF was much lower at 6\% (95\% CI -.06 to .16). The PAF for maltreatment in predicting CANSAS safety-to-others was $23 \%$ (95\% CI .01 to .40).

The PAF for CANSAS safety-to-others in predicting both hostility $(-.3 \%$; 95\% CI -.05 to .04) and high hostility (1.3\%; 95\% CI-.08 to .05) was negligible. For maltreatment in predicting hostility, the PAF was $1.7 \%$ (95\% CI -.06 to .09) and for high hostility $3.2 \%$ (95\% CI -.09 to .14).

For maltreatment, 5.6\% (95\% CI -.06 to .16) was attributable to hostility and $-.3 \%$ (95\% CI -.07 to .06) to high hostility. 9.3\% (95\% CI .00 to .17) of maltreatment was attributable to CANSAS safety-to-others. 
Table 3: Risk factors for other safety need, hostility, high hostility and maltreatment to others

\begin{tabular}{|c|c|c|c|c|}
\hline & $\begin{array}{l}\text { CANSAS safety- } \\
\text { to-others (met or } \\
\text { unmet need) }\end{array}$ & $\begin{array}{l}\text { PANSS hostility } \\
\quad(\text { score }>1)\end{array}$ & $\begin{array}{l}\text { PANSS high hostility } \\
\quad(\text { score }>2)\end{array}$ & $\begin{array}{c}\text { Maltreatment to } \\
\text { others }\end{array}$ \\
\hline & $\begin{array}{l}\text { Hazard ratio } \\
(95 \% \mathrm{CI})\end{array}$ & $\begin{array}{l}\text { Hazard ratio } \\
(95 \% \mathrm{CI})\end{array}$ & $\begin{array}{c}\text { Hazard ratio } \\
(95 \% \mathrm{CI})\end{array}$ & $\begin{array}{c}\text { Hazard ratio } \\
(95 \% \mathrm{CI})\end{array}$ \\
\hline $\begin{array}{l}\text { Demographic items } \\
\text { Age }\end{array}$ & $.95(.89-1.01)$ & $.99(.96-1.02)$ & $.98(.94-1.02)$ & $.89(.84-.93) * * *$ \\
\hline Sex & $.21(.05-.90)^{*}$ & $.58(.34-.99)^{*}$ & $.73(.36-1.45)$ & $.51(.25-1.04)$ \\
\hline Ethnicity & $.58(.20-1.68)$ & $.87(.51-1.47)$ & $1.01(.52-1.99)$ & $1.42(.79-2.55)$ \\
\hline $\begin{array}{r}\text { Education } \\
\text { No/primary } \\
\text { Lower secondary } \\
\text { Lower vocational } \\
\text { Higher secondary } \\
\text { Higher vocational } \\
\text { University }\end{array}$ & $\begin{array}{c}12.04(1.40- \\
103.28)^{*} \\
2.95(.36-24.31) \\
3.49(.44-27.58) \\
2.45(.29-20.64) \\
1.50(.14-16.71) \\
1\end{array}$ & $\begin{array}{c}3.99(1.32-12.00)^{*} \\
1.97(.75-5.15) \\
1.99(.77-5.18) \\
2.11(.80-5.57) \\
1.43(.50-4.13) \\
1\end{array}$ & $\begin{array}{c}4.64(.92-23.34) \\
2.58(.59-11.33) \\
2.48(.57-10.82) \\
2.23(.50-9.97) \\
1.12(.20-6.16) \\
1\end{array}$ & $\begin{array}{c}4.64(.96-22.33) \\
1.81(.41-7.90) \\
1.40(.32-6.20) \\
1.87(.43-8.19) \\
1.44(.29-7.18) \\
1\end{array}$ \\
\hline Maltreatment to others & $\begin{array}{l}6.28(2.69- \\
14.64) * * *\end{array}$ & $1.88(1.02-3.46)^{*}$ & $2.42(1.17-4.99) *$ & \\
\hline CAPE Suicidality & $1.86(1.26-2.75)^{* *}$ & $1.36(1.07-1.74)^{*}$ & $1.46(1.07-2.01)^{*}$ & $1.82(1.36-2.43)^{* * *}$ \\
\hline Suicide attempts & $4.15(1.61-10.68)^{* *}$ & $1.33(.68-2.59)$ & $.60(.18-1.96)$ & $2.27(1.19-4.33)^{*}$ \\
\hline Baseline IQ & $1.00(.98-1.03)$ & $1.00(.99-1.02)$ & $.99(.97-1.01)$ & $.99(.97-1.01)$ \\
\hline CTQ $80^{\text {th }}$ percentile & $.98(.45-2.13)$ & $1.65(1.08-2.50)^{*}$ & $1.85(1.03-3.33)^{*}$ & $1.74(1.03-2.93)^{*}$ \\
\hline PAS & $1.11(.72-1.69)$ & $1.16(.92-1.46)$ & $.95(.69-1.30)$ & $1.18(.87-1.61)$ \\
\hline $\begin{array}{l}\text { Cannabis use in the } \\
\text { past } 12 \text { months }\end{array}$ & $2.04(.98-4.26)$ & $1.79(1.19-2.68)^{* *}$ & $2.16(1.26-3.70)^{* *}$ & $2.74(1.64-4.57)^{* * *}$ \\
\hline $\begin{array}{l}\text { CANSAS number of } \\
\text { unmet needs }\end{array}$ & $1.30(1.17-1.45)^{* * *}$ & $1.17(1.10-1.26)^{* * *}$ & $1.12(1.02-1.23) *$ & $1.28(1.18-1.40)^{* * *}$ \\
\hline $\begin{array}{l}\text { CANSAS } \\
\text { Safety-to-self }\end{array}$ & $\begin{array}{c}15.32 \\
(7.50-31.32)^{* * *}\end{array}$ & $\begin{array}{c}.69 \\
(.28-1.69)\end{array}$ & $\begin{array}{c}.81 \\
(.29-2.22)\end{array}$ & $\begin{array}{c}1.42 \\
(.62-3.26)\end{array}$ \\
\hline Safety-to-others & & $\begin{array}{c}3.58 \\
(1.69-7.62)^{* * *}\end{array}$ & $\begin{array}{c}5.43 \\
(2.44-12.09)^{* * *}\end{array}$ & $\begin{array}{c}4.50 \\
(1.96-10.34)^{* * *}\end{array}$ \\
\hline
\end{tabular}

\begin{tabular}{lcccc} 
PANSS & & & & \\
Hostility (continuous) & $1.56(1.03-2.34)^{*}$ & & & $1.07(.69-1.65)$ \\
Excitement & $1.11(.71-1.74)$ & $1.31(1.04-1.66)^{*}$ & $1.60(1.17-2.20)^{* *}$ & $1.26(.84-1.89)$ \\
Tension & $1.11(.79-1.55)$ & $1.16(.96-1.40)$ & $1.06(.81-1.38)$ & $1.03(.77-1.37)$ \\
Uncooperativeness & $.53(.31-.91)^{*}$ & $1.63(1.32-2.02)^{* * *}$ & $1.65(1.24-2.18)^{* * *}$ & $1.01(.63-1.63)$ \\
Poor impulse control & $2.38(1.56-3.64)^{* * *}$ & $1.59(1.25-2.03)^{* * *}$ & $1.19(.85-1.67)$ & $1.44(.92-2.23)$ \\
\hline
\end{tabular}

$* P<0.05 ; * * P<0.01 ; * * * P<0.001 ;$ a:reference category; Grey areas encompass variables that were included models; all models and items are adjusted for age and sex;PAS, Premorbid Adjustment Scale; CTQ, Childhood Trauma Questionnaire dichotomized with the cut-off at the $80^{\text {th }}$ percentile of scores for the healthy control subjects; CAPE, community assessment of psychic experiences; CANSAS, Camberwell Assessment of Need Short Appraisal Schedule; PANSS, Positive and Negative Syndrome Scale. 


\section{Additional analysis}

In addition to the aim and scope of this paper, some additional analyses were done to examine the strong association of CANSAS safety-to-others with CANSAS safetyto-self and with poor impulse control. For CANSAS safety-to-self, we conducted a survival analysis, a regression analysis and the calculated the PAF.

There were 62 failures of CANSAS safety-to-self in single failure per subject data and the yearly incidence was $1.6 \%$. The yearly incidence rate for CANSAS safetyto-self in patients with a CANSAS safety-to-others was $10 \%$ versus $1.4 \%$ in patients without a CANSAS safety-to-others. The yearly incidence of CANSAS safety-to-self was not increased for patients with $(1.3 \%)$ and without (1.7\%) hostility. The same applied to patients with $(1.5 \%)$ and without (1.5\%) maltreatment. For patients with poor impulse control, the yearly incidence of CANSAS safety-to-self was $2.6 \%$ versus $1.5 \%$ in patients without poor impulse control. For CANSAS safety-to-others, the yearly incidence in patients with poor impulse control was 3.4\% versus .5\% in patients without poor impulse control.

CANSAS safety-to-self was significantly associated with CANSAS safety-toothers (HR $6.89 p=.00)$. In the COX regression model of CANSAS safety-to-self with the PANSS items, there were significant associations with poor impulse control (HR $1.73 p=.02)$ and tension (HR $1.32 p=.03)$ but not with hostility (HR .69 $p=.17)$, excitement (HR .83 $p=.38)$ and uncooperativeness (HR $1.27 p=.26)$.

The PAF for poor impulse control in predicting CANSAS safety-to-self was 0.6\% (95\% CI -.09 to .09) while 26\% (95\% CI .05 to .43) of CANSAS safety-toothers was attributable to poor impulse control. The PAF for CANSAS safety-toothers in predicting CANSAS safety-to-self was $-1.6 \%$ (95\% CI -.06 to .03) whereas vice versa, $13 \%$ (95\% CI -.03 to .26) of CANSAS safety-to-others was attributable to CANSAS safety-to-self.

\section{Discussion}

This paper is, to our knowledge, the first survival analysis of hostile behaviours, self-reported incidents of aggression and associated care needs. Because of the use of these different measures, results provide insight in data on self-reported incidents of aggression (maltreatment), symptoms (PANSS hostility) and associated care needs (CANSAS safety-to-others). We found that the incidence of these behaviours in this cohort is low and largely confirm previous studies considering risk factors such as male sex, lower educational level, suicidal ideation, cannabis use and more CANSAS unmet needs. Most known risk factors apply also for care needs associated with safety for other people. Childhood adversity was associated with hostility and maltreatment, 
but not with CANSAS safety-to-others. Premorbid adjustment problems were not associated with aggression towards others. Persistence rates were low for all outcome measures, although around one in five patients present themselves three years later with the same outcome. Moreover, results of the PAF analysis indicate that, assuming causality, both CANSAS safety-to-others and maltreatment can be traced back to hostile and impulsive behaviour at an earlier visit, although the preventive potential is not very high.

The incidence for CANSAS safety-to-others, hostility and maltreatment is lower compared with meta-analytic data (Fazel et al., 2009; Iozzino et al., 2015; Witt et al., 2013). However, these rates are often difficult to compare due to the differences in populations (for example: outpatients, acutely ill inpatients or forensic populations), the use of different definitions of violence and aggression (varying from verbal aggression to homicide) and differences in data collection (for example:interview-based,incidents reports or legal data). The current study used mostly interview-based data. This should be valid and reliable for measuring violence incidence in adult mental health care (Johnson, Desmarais, Tueller, \& Van Dorn, 2019). There is, however, no objective data to confirm if these data is reliable and there could be an underestimation because of the 3-year time-intervals between the visits.

There are some differences between the outcome measures throughout the analysis. This could be because of the different references periods. While the maltreatment item was aimed at lifetime problems (baseline) or in the past three years (follow-up), the PANSS was aimed at symptoms that occurred in the past week and the CANSAS reference period was the past month. This difference in reference periods is also why patients reported more maltreatment incidents than CANSAS care needs.

The results on risk factors largely confirm previous studies reporting a link between hostility and aggressive behaviour with male sex, lower education, cannabis use and more CANSAS unmet needs (Hodgins \& Klein, 2017; Large \& Nielssen, 2011; Martinez-Martin et al., 2011; Witt et al., 2013). IQ was not associated with the outcome measures, which is consistent with the results of Witt et al. (2013).

Premorbid adjustment problems were not associated with the outcome measures. Only one previous study, as far as we are aware, analysed premorbid adjustment, reporting a significant association between violent behaviour and some factors on the PAS, such as peer relationships, but not all (Fresán et al., 2004). Childhood trauma was linked to hostility and maltreatment, but not to CANSAS safety-to-others. Previous studies found an association between childhood adversity and aggressive behaviour in patients with psychosis, although the strongest association may be with sexual, and not with physical abuse (Bosqui et al., 2014; Swanson et al., 2006; Swanson et al., 2008; Witt et al., 2013). Another study using the current dataset found hostile behaviours to be 
on the route from childhood adversity to psychosis (Isvoranu et al., 2017). Childhood problems are one of the predisposing factors on the possible causal pathways leading to aggression (Volavka \& Citrome, 2011). The complex relation between childhood adversity, conduct disorders and violent behaviour in schizophrenia may include shared risk factors on a path leading to both violence and schizophrenia (Oakley, Harris, Fahy, Murphy, \& Picchioni, 2016).

Impulsivity has previously been associated with violence incidents (Moulin, Golay, et al., 2018; Witt et al., 2013) although we only found an association with hostility and CANSAS safety-to-others and not with maltreatment and high hostility. Another relevant finding was that, assuming causality, $26 \%$ of the CANSAS safetyto-others failures could be traced back to poor impulse control three years earlier while only $18 \%$ of the CANSAS safety-to-others failures could be explained by hostility at the previous visit. Moreover, for uncooperativeness, there was an increased risk for hostility but a decreased risk for CANSAS safety-to-others. Possibly, the association with hostility is simply because higher scores on the PANSS item lack of cooperation include hostile attitudes or behaviours (Kay et al., 1987). A similar reasoning may apply to excitement, higher scores of which includes agitation, which was significantly associated with hostility but not with CANSAS safety-to-others nor with maltreatment.

Aggressive behaviours towards self and others are interrelated. Previous work found an association between violence risk and suicidal threats, but not with suicidal attempts (Witt et al., 2014; Witt et al., 2013). Witt et al point out that impulsivity could be the mediator between suicidality and violence and thereby explain why suicidal behaviours are more strongly associated with violence than suicidal ideation (Witt et al., 2014). The current results also indicate a strong relation between impulsivity, suicidality and aggressive behaviour. There are, however, differences between the outcome measures and there is a lack of association of CANSAS safety-to-self with hostility and maltreatment when corrected for CANSAS safety-to-others.

This study has several limitations. The GROUP study was not specifically designed to measure violence incidents. Although we used three different outcome measures as indicators for aggression, the maltreatment item relies on self-report with long time intervals. Patients may have forgotten incidents that occurred between the visits. Moreover, maltreatment was assessed with one question only. We did not have access to incident reports or data pertaining to the criminal justice system. Drop-out during follow-up could be selective. Patients with aggressive behaviour could be less likely to participate with interviews or are, for example, incarcerated or admitted to a closed ward. Second, the results of our PAF analysis should be interpreted with some caution. The PAF assumes causality, which is for the current outcome measures not as clear as with, for example, the PAF of smoking in relation to lung cancer. Third, 
PANSS scores of 2 indicate "questionable pathology; may be at the upper extreme of normal limits"(Kay et al., 1987) while we label a score of 2 as "hostile." We therefore used "high hostility" with PANSS scores $>2$ as the primary outcome measure, next to the, rather sensitive, measure of hostility. The strength of the current study is its prospective, long-term nature and the large sample size, which allowed for a survival analysis.

Several implications for clinical practice can be mentioned. Importantly, the incidence of indicators of aggressive behaviour was very low. In addition, a number of known risk factors for mostly prevalent aggressive behaviour were confirmed for incidence measures, which could help in detecting patients at risk. The strong association between CANSAS safety-to-others and safety-to-self stands out, as do associations with treatable psychopathology. At least part of the CANSAS safetyto-others was attributable to previous hostile and impulsive behaviour. Under the assumption of causality, if these behaviours been successfully treated three years earlier, the CANSAS safety-to-others may have never occurred. This indicates the importance for clinicians to anticipate on these behaviours in order to prevent future problems in the domain of safety for others, although the preventive potential is not very high.

While the current study contributes to knowledge about aggressive behaviour, it remains unclear why some of the outcome measures have significant associations with known risk factors while others have not. Future research should focus on the mechanisms leading to violence and aggression, including the relation with impulsivity and suicidal behaviour. Ideally, there should be a combination of self-report, family and clinical observations and legal data.

\section{Conclusion}

In conclusion, this paper is, to our knowledge, the first survival analysis of hostile behaviours, self-reported incidents of aggression and associated care needs. Many of the known risk factors for prevalence also apply to incidence and for care needs associated with safety for other people. There were, however, differences between the outcome measures. Violence and aggression in patients with psychosis appears to be a complex concept with different origins, as this and other studies have shown. We found childhood adversity but not premorbid problems to be associated with aggressive behaviour. Clinical symptoms can index aggressive behaviour and associated care needs years later, providing opportunities for preventive strategies and a possible decrease of incidents of aggression. 



\section{CHAPTER 5}

Hostility and aggressive behaviour in first episode
psychosis: results from the OPTiMiSE trial

Margo Faay

Caroline van Baal

Celso Arango

Covadonga Díaz-Caneja

Gregor Berger

Stefan Leucht

Julio Bobes

Pilar Sáiz

María Paz García-Portilla

Resy van de Brug

Jocelyn Petter

Inge Winter-yan Rossum

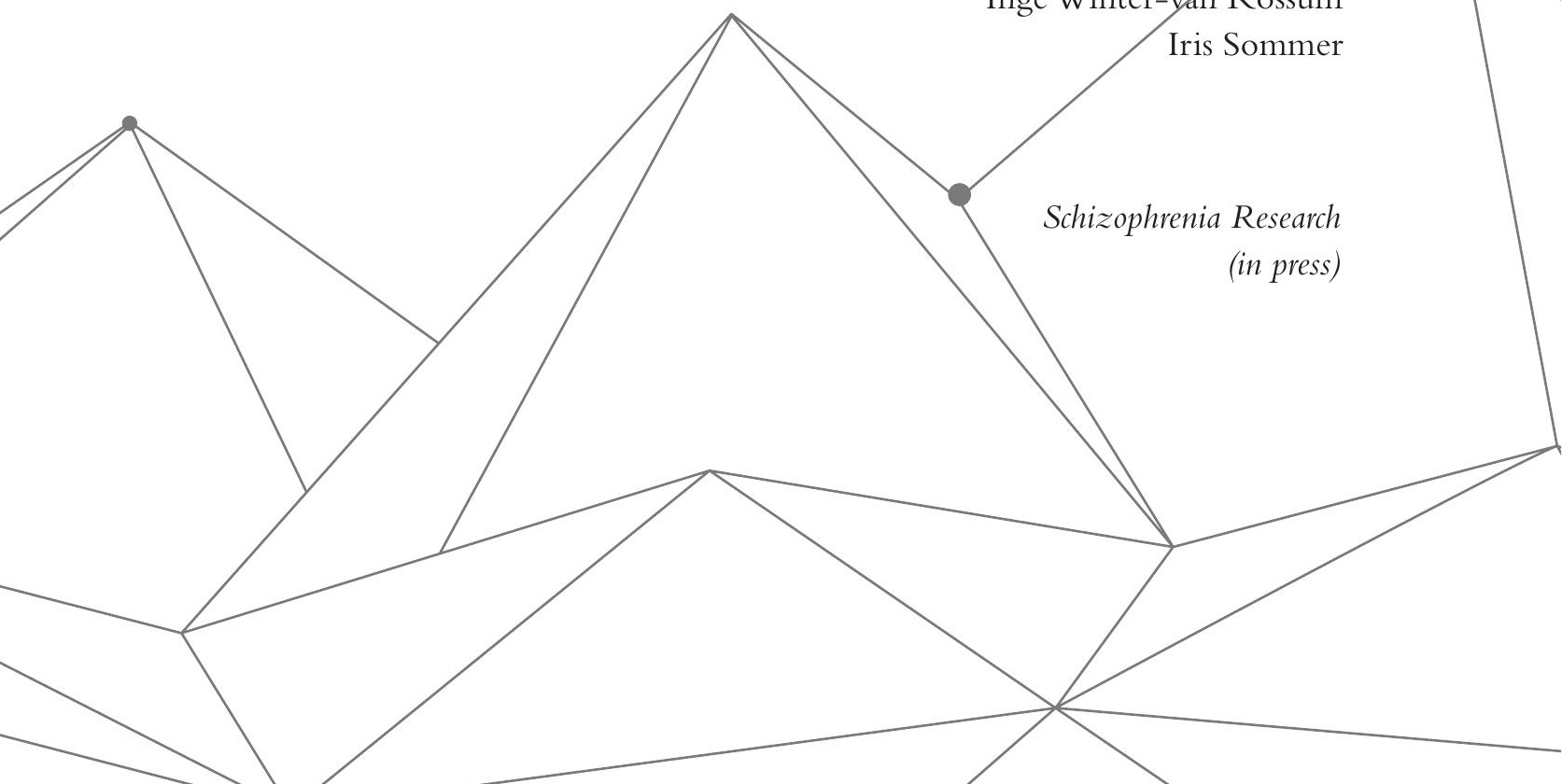


The aim of this paper is to determine clinical factors related to hostility and disturbing and aggressive behaviour and to examine the effect of medication on these behaviours in FEP.

Data from phase I and II of the Optimise trial are used. Outcome measures are the hostility item of the Positive and Negative Syndrome Scale (PANSS P7) and the disturbing and aggressive behaviour domain of the Personal and Social Performance scale (PSP-D).

Moderate, severe or extreme hostility (PANSS P7 >3) was present in 42 patients (9.4\%). The PANSS P7 and PSP-D were low to moderate but significantly associated with the selected PANSS items: delusions, hallucinatory behaviour, excitement, tension, uncooperativeness, unusual thought content, impulsivity, and lack of judgement and insight. In a subsample of 185 patients (41.5\%) with baseline PANSS P7 $>1$, the PANSS P7 and PSP-D scores improved in the first 4 weeks of amisulpride treatment. This effect remained significant after controlling for baseline positive symptoms (PANSS P1-P6). No significant differences were found between olanzapine and amisulpride in the second phase of the trial.

Clinical risk factors such as poor impulse control, uncooperativeness and excitement could help clinicians in detecting and treating hostile and aggressive behaviour in FEP. Amisulpride could be an effective antipsychotic choice in the treatment of FEP patients who express hostile or aggressive behaviour. Future research is needed to compare the effects of amisulpride and olanzapine on hostility in FEP during the first weeks of treatment.

\section{ABSTRACT}




\section{Introduction}

A considerable proportion of patients with first episode psychosis (FEP) are involved in aggressive incidents. Studies estimate that around 30\% of patients commit at least one violent act during their first psychotic episode (Large \& Nielssen, 2011; Winsper et al., 2013). Aggression often leads to a number of negative consequences for the patients as well as their immediate social network, health care workers and the public, such as prolonged hospitalisation or social isolation (Lahera et al., 2015; Volavka, 2008).

For clinicians, it is essential to have some knowledge of the factors that are associated with hostile or aggressive behaviour, as this enables risk assessment and treatment planning. Clinical risk factors for hostile or aggressive behaviour in psychotic disorders in general include previous violence, symptoms of mania, substance abuse, treatment non adherence, excitement, impulsivity, lack of insight and positive symptoms (Arango, 2000; Arango et al., 1999; Bobes et al., 2009; Faay, van Os, \& Genetic Risk and Outcome of Psychosis Investigators, 2020; Fazel et al., 2009; Volavka et al., 2016; Witt et al., 2013). Specifically for FEP, a meta-analysis identified risk factors for any type of violence, which included younger age, male sex, substance abuse, manic symptoms, lower level of education and longer duration of untreated psychosis (Large \& Nielssen, 2011). While positive symptoms are a risk factor for aggressive behaviour in more chronic psychotic disorders, the findings within FEP populations are inconsistent. In contrast to meta-analytic data (Large \& Nielssen, 2011), a 10-year follow-up study of FEP found higher positive symptom scores in patients with violent behaviour (Langeveld et al., 2014). More specifically, certain positive symptoms, such as delusions and uncommon beliefs were associated with violence in FEP (Coid et al., 2013) but a recent study in an ultra-high-risk sample found no significant relation between delusions, unusual thought content, hallucinations and violent behaviour (Brucato et al., 2019). Next to positive symptoms, clinical risk factors that are observed from patients' behaviour could be important for risk management. Impulsivity is associated with violent behaviour in early psychosis (Moulin, Baumann, et al., 2018; Moulin, Golay, et al., 2018). In patients with more chronic psychotic disorders, uncooperativeness and excitement were related to hostile and aggressive behaviour next to impulsiveness (Faay et al., 2020). However, as far as we are aware, there are no studies examining the association between symptoms such as uncooperativeness, excitement and tension with aggressive behaviour in FEP.

Although violence, aggression and hostility are different, hostile behaviour can be seen as a risk factor for violent or aggressive acts since this behaviour is often observed prior to incidents (Large \& Nielssen, 2011; Witt et al., 2013). Moreover, since most major studies do not include a separate measure of violence or aggression, the hostility item of the Positive and Negative Syndrome Scale (PANSS P7 (Kay 
et al., 1987)) is often used as a proxy to give an indication of the level of aggressive behaviour.

Some randomised trials presented post-hoc analysis of the PANSS hostility item. The European First-Episode Schizophrenia Trial (EUFEST) compared five antipsychotic drugs in 498 patients with first episode schizophrenia, schizoaffective disorder or schizophreniform disorder in terms of efficacy and tolerability. In a subset of 302 patients with at least minimal hostility $(>1)$ at baseline, olanzapine was superior to other antipsychotics in reducing hostility (Volavka et al., 2011). A similar result was found in the Clinical Antipsychotic Trials of Intervention Effectiveness (CATIE) study including 1493 patients with schizophrenia. Post-hoc analyses of this study found olanzapine to be significantly superior in reducing hostility compared with perphenazine, quetiapine, ziprasidone and risperidone (Volavka, Czobor, Citrome, \& Van Dorn, 2014). A recent meta-analysis, including these trials, compared the effect of antipsychotics on hostility, in 18 randomized studies. A small but significant effect was found for second-generation as compared to first-generation antipsychotics (Faay, Czobor, \& Sommer, 2018). Especially clozapine was superior, in line with previous studies (Frogley et al., 2012; Glazer \& Dickson, 1998; Spivak, Mester, Wittenberg, Maman, \& Weizman, 1997). Current clinical guidelines recommend clozapine for patients who do not respond to two adequate trials with different antipsychotics (Warnez \& Alessi-Severini, 2014). Thus, FEP patients can only start with clozapine after several weeks of treatment. Evidence considering the effectiveness of other second-generation antipsychotics on hostile and aggressive behaviour in the first weeks of treatment is therefore needed.

The present paper focuses on the PANSS P7 hostility item and the PSP-D disturbing and aggressive behaviour domain in the Optimization of Treatment and Management of Schizophrenia in Europe (OPTiMiSE) trial. We aim to determine clinical factors related to hostility and disturbing and aggressive behaviour, and to examine the effect of medication on these behaviours in FEP patients. To examine the effect of medication, there is a two-fold aim: 1) to describe the short-term effect of amisulpride on hostility and disturbing and aggressive behaviour and 2) to compare the effect of continuation of amisulpride versus switching to olanzapine on hostility and disturbing and aggressive behaviour in patients who were not in remission after four weeks of treatment with amisulpride. 


\section{Methods}

\section{OPTiMiSE trial}

The rationale and design of the OPTiMiSE trial is described elsewhere (Leucht et al., 2015). A total of 481 patients with first episode schizophrenia, schizophreniform or schizoaffective disorder were included in 27 sites across European countries and Israel. In phase I, patients received open label amisulpride (200-800 mg/d). Patients who were not in remission after 4 weeks continued in phase II of the trial and were randomized to 6 weeks double blind treatment with either a continuation of amisulpride or a switch to olanzapine $(5-20 \mathrm{mg} / \mathrm{d})$. If these patients still not reached remission criteria at week 10, they entered phase III where they received 12 -weeks open label clozapine (100-900 mg/d). Dosing was flexible in all three phases of the trial. For the current analysis, data from phase I and II are used. Each country obtained regulatory approval. All participants provided written informed consent. A data safety monitoring board oversaw the study.

\section{Measures}

The Positive And Negative Syndrome Scale (PANSS (Kay et al., 1987)), was used to assess the severity of clinical symptoms associated with psychosis. All 30 PANSS items are scored from absent (1) to severe impairment (7), resulting in a total score between 30 and 210. The primary outcome measure for the current analysis is the P7 hostility item, an item on the positive subscale. Hostility is defined as "verbal and nonverbal expressions of anger and resentment, including sarcasm, passive-aggressive behaviour, verbal abuse and assaultiveness" (Kay et al., 1987). The score is based on interpersonal behaviour during the interview and reports by primary care workers or family during the past week. A score of 1 indicates absent hostility, a score of 2 indicates minimal presence of hostility (questionable pathology), 3 is labelled as mild, 4 is moderate, 5 indicates moderate-severe, 6 is severe and a score of 7 indicates extreme, including marked anger, resulting in physical assault towards others.

Next to the PANSS, the Personal and Social Performance Scale (PSP (Morosini, Magliano, Brambilla, Ugolini, \& Pioli, 2000) was used. The PSP consists of four main domains of functioning: A) social useful activities, B) personal and social relationships, C) self-care and D) disturbing and aggressive behaviour. All domains are scored on a 6-point scale which has a specific description of the degrees of severity for the "disturbing and aggressive behaviour" domain. This domain is based on behaviour during the past month and scored from absent (1) to very severe (6), indicating "frequent aggressive acts, aimed at, or likely to cause severe injuries." 
For the analysis of clinical factors associated with hostility, we selected seven PANSS items to see if these are related to PANSS P7 and PSP-D. We included the four PANSS items that are in the Excitement Component (PANSS-EC), along with hostility: P4 excitement, G14 poor impulse control, G4 tension and G8 uncooperativeness (Montoya et al., 2011). Impulsivity was associated with violence in FEP (Moulin, Golay, et al., 2018) and more chronic populations (Faay et al., 2020), but for excitement, tension and uncooperativeness, there is lacking evidence in a population of FEP. Next to these items, we wanted to examine whether delusions and hallucinations are associated with hostility or aggression because of the contrasting results from previous studies (Brucato et al., 2019; Coid et al., 2013; Langeveld et al., 2014; Large \& Nielssen, 2011). We therefore included P1 delusions, P3 hallucinatory behaviour, and G9 unusual thought content, as this last item is associated with the first two.

Other measures used to describe the current sample include demographic data, type of care, duration of current psychosis, use of antipsychotics before inclusion to the trial and Clinical Global Impression (CGI (Guy, 1976)). Diagnosis was measured with the Mini International Neuropsychiatric Interview Plus (M.I.N.I. Plus (Sheehan et al., 1998)).All questionnaires and assessments were conducted by members of the local study team who were trained in the procedures and questionnaires. For administration of the PANSS, a standardised training was developed that included examination.

\section{Statistical procedures}

\section{Clinical factors}

To assess the clinical factors related to hostility (PANSS P7) and disturbing and aggressive behaviour (PSP-D) in a cross-sectional fashion, baseline values from the intention-to-treat sample were used to calculate Spearman's rank-order correlation $\left(r_{\mathrm{s}}\right)$. In general, correlation coefficients of .00 to .30 are considered negligible, .30 to .50 low, .50 to .70 moderate and $>.70$ high to very high (Hinkle, Wiersma, \& Jurs, 1988). This is, however, dependent of the context. For example a correlation of $>.70$ should be expected for instruments while correlations of a psychosocial nature rarely exceed .50 (Polit \& Lake, 2010).

\section{Phase I}

In line with the hostility analysis of the CATIE and EUFEST studies, a subset of patients with a baseline PANSS P7 hostility score of at least 2 is used (i.e. PANSS P7 $>1$ ), since there is no improvement possible in patients without baseline hostility.

For phase I, raw data were visualized using histograms, and mean values and $S D$ of differences between baseline values of PANSS-P7/PSP-D and values at weeks 
1, 2 and 4 were summarized in tables. These differences with their 95\% Confidence Intervals $(C I)$ were also visualized in high-low plots.

A general linear mixed model analysis was performed to test the differences in scores on PANSS-P7 at week 1, 2 and 4 (main effect and interaction with visit). Analyses were controlled for baseline phase I hostility, baseline phase I PANSS positive items P1-P6 (without hostility), and visit. Similar analyses were performed for PSP-D. General linear mixed models were chosen because these incorporate all available measurements and can handle missing values, assuming that these are missing at random.

\section{Phase II}

For the second phase, a new selection was made based upon hostility scores at the baseline phase II visit in week 4. For phase II, ITT analyses were performed. For both randomized groups, data were visualized using high-low plots, and mean values and $S D$ of differences between baseline values of PANSS-P7/PSP-D and values at weeks 6,8 and 10 were summarized in tables.

Again, general linear mixed models were used to test the effect of randomized treatment (continuation of amisulpride vs. switching to olanzapine) on PANSS-P7/ PSP-D values at week 6,8 and 10 (main effect and interaction with visit), controlling for baseline phase II hostility, baseline phase II PANSS positive items P1-P6 (without hostility) and visit. SPSS version 25 was used for all analyses.

\section{Results}

\section{Sample characteristics}

A total of 446 patients comprised the intention-to-treat sample. Baseline characteristics of this sample are described elsewhere (Kahn et al., 2018). From these 446 patients, 185 $(41.5 \%)$ had a baseline PANSS hostility score $>1$ and were included in the hostility subsample. Of this subsample, most patients had minimal $(35.1 \%)$ or mild $(42.2 \%)$ hostility. A total of 210 patients (47\%) of the intention-to-treat sample scored on the PSP-D at baseline. Manifest, marked, severe or very severe disturbing or aggressive behaviour was present in $90(20.2 \%)$ patients. 127 patients $(28.5 \%)$ had both a hostility and PSP-D score. 178 patients (39.9\%) scored on neither of these scales. 58 patients $(13 \%)$ of the intention-to-treat sample had a hostility score but no PSP-D. Baseline characteristics of the hostility subsample are found in Table 1. 
Table 1: Baseline characteristics of patients with baseline PANSS P7 hostility $>1$

\begin{tabular}{|c|c|c|c|c|}
\hline & & $\begin{array}{c}\text { Phase } 1 \\
n=185 \\
N(\%)\end{array}$ & $\begin{array}{c}\text { Phase } 2 \text { AMI } \\
n=16 \\
N(\%)\end{array}$ & $\begin{array}{c}\text { Phase } 2 \text { OLA } \\
n=12 \\
N(\%)\end{array}$ \\
\hline Age, years (mean; SD) & & $25.2(5.7)$ & $25.5(5.7)$ & $23.0(4.8)$ \\
\hline \multicolumn{5}{|l|}{ Sex } \\
\hline & Women & $65(35.1 \%)$ & $3(18.8 \%)$ & $1(8.3 \%)$ \\
\hline & Men & $120(64.9 \%)$ & $13(81.3 \%)$ & $11(91.7 \%)$ \\
\hline
\end{tabular}

Race

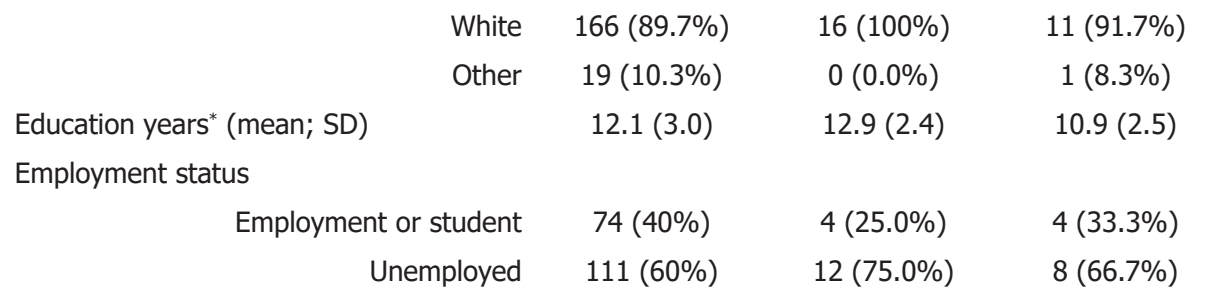

Disease type ${ }^{+}$

$$
\begin{array}{r}
\text { Schizophreniform disorder } \\
\text { Schizoaffective disorder } \\
\text { Schizophrenia }
\end{array}
$$

Comorbid major depressive disorder

Suicidality"

Substance abuse or dependence in the past 12 months

Type of care baseline

$$
\begin{array}{r}
\text { Inpatient } \\
\text { Outpatient }
\end{array}
$$

Duration of current psychosis, months

(mean; SD)

Antipsychotic naïve at baseline

CGI severity (mean; SD)

Clinical scores (mean; SD)

$\begin{array}{ccc}76(41.1 \%) & 5(31.3 \%) & 4(33.3 \%) \\ 12(6.5 \%) & 0(0.0 \%) & 1(8.3 \%) \\ 97(52.4 \%) & 11(68.7 \%) & 7(58.3 \%) \\ 15(8.6 \%) & 1(6.7 \%) & 0(0.0 \%) \\ 26(14.9 \%) & 1(6.7 \%) & 3(25 \%) \\ 40(23 \%) & 1(6.7 \%) & 5(41.7 \%)\end{array}$

$\begin{array}{lll}114(61.6 \%) & 9(56.3 \%) & 4(33.3 \%) \\ 71(38.4 \%) & 7(43.8 \%) & 8(66.7 \%)\end{array}$

$\begin{array}{ccc}6.8(0.47) & 9.13(7.8) & 9.8(7.5) \\ 81(43.8 \%) & 10(62 \%) & 9(75 \%) \\ 5.7(0.9) & 5.4(0.9) & 5.7(0.6)\end{array}$

PANSS total

85.9 (17.8)

$95.9(20.8)$

81.4 (10.6)

PANSS positive

$23.1(5.1)$

$25.2(6.5)$

$23.0(4.4)$

PANSS negative

20.7 (6.9)

$24.9(8.1)$

$22.7(7.0)$

PANSS general

45.7 (11.0)

$42.1(9.6)$ 
PANSS P7 hostility

$\begin{array}{rccc}\text { 2- Minimal } & 65(35.1 \%) & 4(25 \%) & 1(8.3 \%) \\ \text { 3- Mild } & 78(42.2 \%) & 8(50 \%) & 5(41.7 \%) \\ \text { 4- Moderate } & 27(14.6 \%) & 3(18.8 \%) & 3(25 \%) \\ \text { 5- Moderate/severe } & 9(4.9 \%) & 0(0.0 \%) & 2(16.7 \%) \\ \text { 6- Severe } & 3(1.6 \%) & 1(6.3 \%) & 1(8.3 \%) \\ \text { 7- Extreme } & 3(1.6 \%) & 0(0.0 \%) & 0(0.0 \%)\end{array}$

PSP-D

$\begin{array}{rccc}\text { 1- Absent } & 58(32 \%) & 6(42.9 \%) & 2(20.0 \%) \\ \text { 2- Mild } & 61(33.7 \%) & 7(50.0 \%) & 2(20.0 \%) \\ \text { 3- Manifest } & 33(18.2 \%) & 0(0.0 \%) & 2(20.0 \%) \\ \text { 4- Marked } & 17(9.4 \%) & 0(0.0 \%) & 2(20.0 \%) \\ \text { 5- Severe } & 11(6.1 \%) & 1(7.1 \%) & 2(20.0 \%) \\ \text { 6- Very severe } & 1(0.6 \%) & 0(0.0 \%) & 0(0.0 \%)\end{array}$

Data based on sample of patients with baseline hostility $>1$. PANSS=Positive and Negative Syndrome Scale; CGI= Clinical Global Impression; PSP-D Personal and Social Performance Scale domain D: disturbing and aggressive behaviour; $A M I=$ amisulpride; OLA= olanzapine. *years in school from age 6 onwards; + according to the Mini International Neuropsychiatric Interview 5 plus; // suicidality includes medium to high suicide risk; I patients with absent hostility at baseline are not included in the hostility subsample

Table 2: Correlations of PANSS items with PANSS P7 hostility and PSP-D

\begin{tabular}{lll} 
& \multicolumn{2}{c}{$\begin{array}{c}\text { Baseline phase } 1 \\
n=446\end{array}$} \\
& & \\
PANSS items & $\begin{array}{l}\text { PANSS P7 Hostility } \\
\text { Spearman's rho }\end{array}$ & $\begin{array}{l}\text { PSP-D } \\
\text { Spearman's rho }\end{array}$ \\
P1 Delusions & $0.23^{* * *}$ & $0.23^{* * *}$ \\
P3 Hallucinatory Behaviour & $0.13^{* *}$ & $0.15^{* *}$ \\
P4 Excitement & $0.30^{* * *}$ & $0.27 * * *$ \\
P7 Hostility & 1.0 & $0.43^{* * *}$ \\
G4 Tension & $0.19 * * *$ & $0.11^{*}$ \\
G8 Uncooperativeness & $0.43 * * *$ & $0.30^{* * *}$ \\
G9 Unusual thought content & $0.10^{*}$ & $0.19 * * *$ \\
G12 Lack of judgement and insight & $0.25 * * *$ & $0.22 * * *$ \\
G14 Poor impulse control & $0.51 * * *$ & $0.50^{* * *}$ \\
\hline
\end{tabular}

${ }^{*} P<0.05 ; * * P<0.01 ; * * * P<0.001$; based on the complete sample of the OPTIMiSE trial at baseline; PANSS=Positive and Negative Syndrome Scale; PSP personal and Social Performance Scale domain D: disturbing and aggressive behaviours 


\section{Correlations of PANSS items with hostility (PANSS P7) and disturbing and aggressive behaviour (PSP-D)}

In the intention-to-treat sample, PANSS P7 hostility and PSP-D disturbing and aggressive behaviour domain are moderately but significantly associated with each other $(r=.43, p<.0005)$ and with all other selected PANSS items (Table 2). Hostility has the strongest associations with poor impulse control $\left(r_{\mathrm{s}}=.51, p<.0005\right)$, uncooperativeness $\left(r_{\mathrm{s}}=.43, p<.0005\right)$ and excitement $\left(r_{\mathrm{s}}=.30, p<.0005\right)$. Disturbing and aggressive behaviour is associated with poor impulse control $\left(r_{\mathrm{s}}=.50, p<.0005\right)$ and uncooperativeness $\left(r_{\mathrm{s}}=.30, p<.0005\right)$. Gender and duration of psychosis were not significantly correlated with hostility.

\section{Phase I}

The mean PANSS P7 hostility score at baseline for the 185 patients in the hostility subsample was $3.0(S D=1.1$; Table 3$)$, corresponding to mild hostility. At week 4, this was $1.8(S D=1.1)$ corresponding to minimal or absent hostility. Figure 1 shows the distribution of the hostility scores during phase I. At the end of phase I, 10 patients had hostility scores $>3$. The mean change in hostility scores from baseline to week 4 is $-1.3(S D=1.3)$. The mean PSP-D score at baseline is $2.3(S D=1.2)$ corresponding to mild or manifest disturbing and aggressive behaviour and decreased to $1.5(S D=0.9)$ corresponding to absent or mild disturbing and aggressive behaviour at week 4 , a mean change of $-.8(S D=1.2)$. Figure 2 displays the mean change for both hostility and PSP-D.

Primary analysis of phase I hostility controlled for baseline phase I hostility and baseline phase I PANSS Positive items P1-P6, showed a significant reduction over visits $(F=4.111, \mathrm{p}=.017)$. Marginal means were $2.199(95 \% C I=2.040 ; 2.358)$ at week $1,2.119(95 \% C I=1.969 ; 2.269)$ at week 2 , and $1.933(95 \% C I=1.787 ; 2.079)$ at week 4.

\begin{tabular}{|c|c|c|c|c|c|}
\hline & $\begin{array}{l}\text { Baseline } \\
\text { phase I }\end{array}$ & Week 1 & Week 2 & Week 4 & $\begin{array}{l}\text { Baseline to } \\
\text { week } 4\end{array}$ \\
\hline $\begin{array}{l}\text { PANSS P7 hostility } \\
\qquad \text { Mean (SD) }\end{array}$ & $3.0(1.1)$ & $2.4(1.2)$ & $2.1(1.2)$ & $1.8(1.1)$ & \\
\hline Mean difference (SD) & & $-0.7(1.2)$ & $-0.3(1.0)$ & $-0.4(1.0)$ & $-1.3(1.3)$ \\
\hline Mean (SD) & $2.3(1.2)$ & $1.8(1.1)$ & & $1.5(0.9)$ & \\
\hline Mean difference (SD) & & $-0.4(0.9)$ & & $-0.4(0.9)$ & $-0.8(1.2)$ \\
\hline
\end{tabular}

PANSS=Positive and Negative Syndrome Scale; PSP D=Personal and Social Performance Scale domain D: disturbing and aggressive behaviour; data based on the subsample of patients with baseline hostility $>1$. 
Figure 1: Distribution of PANSS P7 hostility scores in patients with baseline hostility $>1$ during phase I

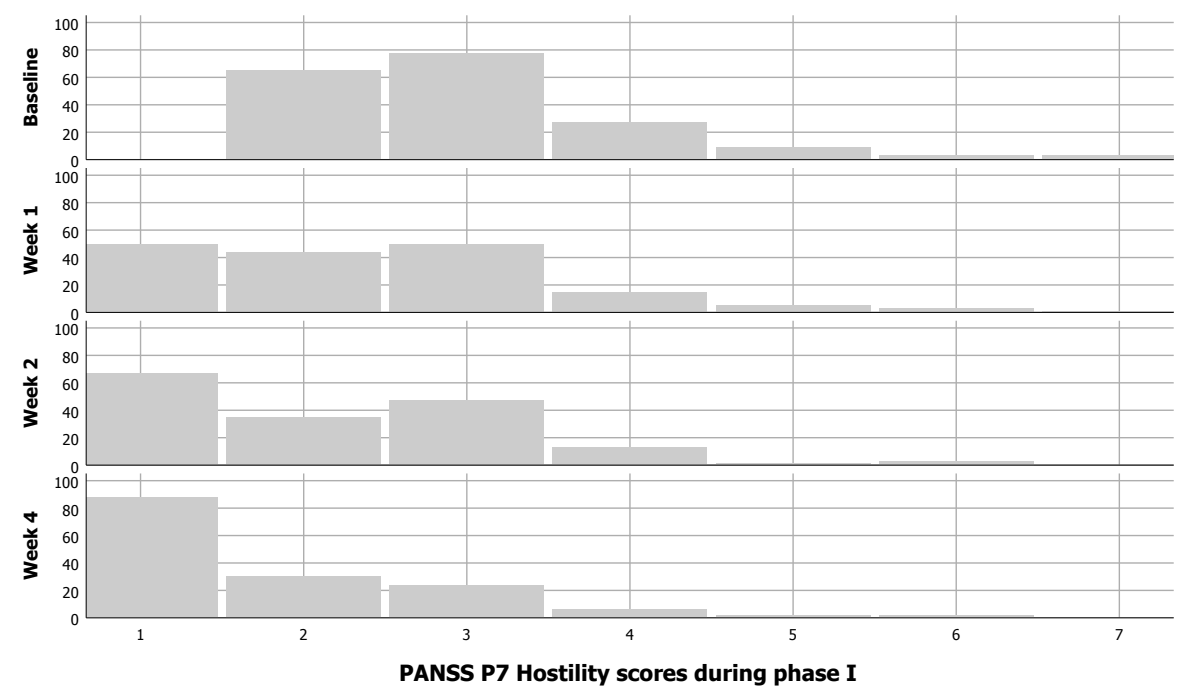

PANSS=Positive and Negative Syndrome Scale; frequencies of PANSS scores in phase I of the trial; based on the subsample of patients with baseline hostility $>1$.

\section{Phase II}

A total of 93 patients continued to the second, double blind phase of the trial. 28 (30.1\%) patients had a PANSS P7 hostility score $>1$ at the baseline phase II visit in week 4 and were included in the hostility subsample of phase II. Of these 28 patients, six had a hostility score $<2$ at baseline phase I. 16 patients were randomized to continue with amisulpride and 12 switched to olanzapine. There are no major differences in baseline characteristics (Table 1). The mean hostility score at baseline phase II for patients with amisulpride was $3.1(S D=1.0$; Table 4$)$ and for olanzapine this was $3.8(S D=1.1)$. At week 10, this was $2.5(S D=1.2)$ for patients with amisulpride and $2.3(S D=1.6)$ for patients with olanzapine. The mean change in phase II was -.7 $(S D=0.8)$ for patients with amisulpride versus $-1.4(S D=1.4)$ for patients with olanzapine (Figure 2).

Controlled for baseline phase II hostility and baseline phase II PANSS Positive items P1-P6, primary analysis of the phase II data showed a trend but no significant reduction in hostility over visits $(F=2.605, p=.087)$. No significant differences between treatment arms were found $(F=1.164, p=.292)$. 


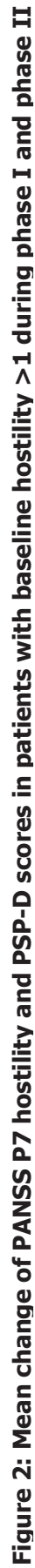

के.
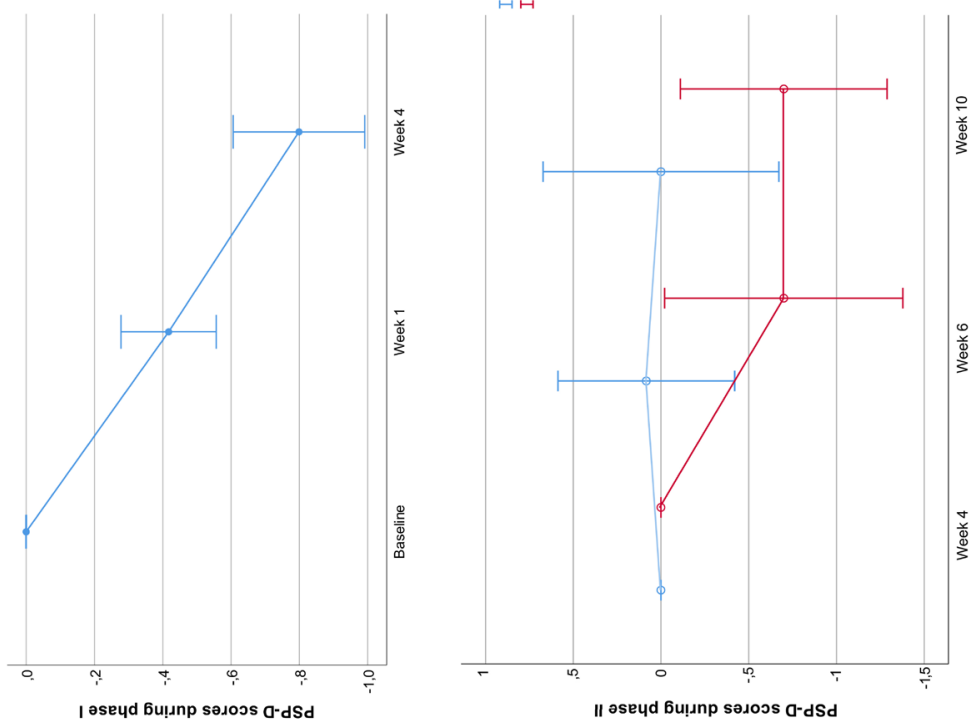

s

कิ

0 จ

ఫ

\& 8

농

ฟิ

ह

बृ

ก.

जह

हิ

$\frac{2}{2}$

论

(8)

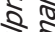

ฟิ

हो के

- $\frac{0}{2}$

४

की

है से

का

इ कू

ชิ ธิ
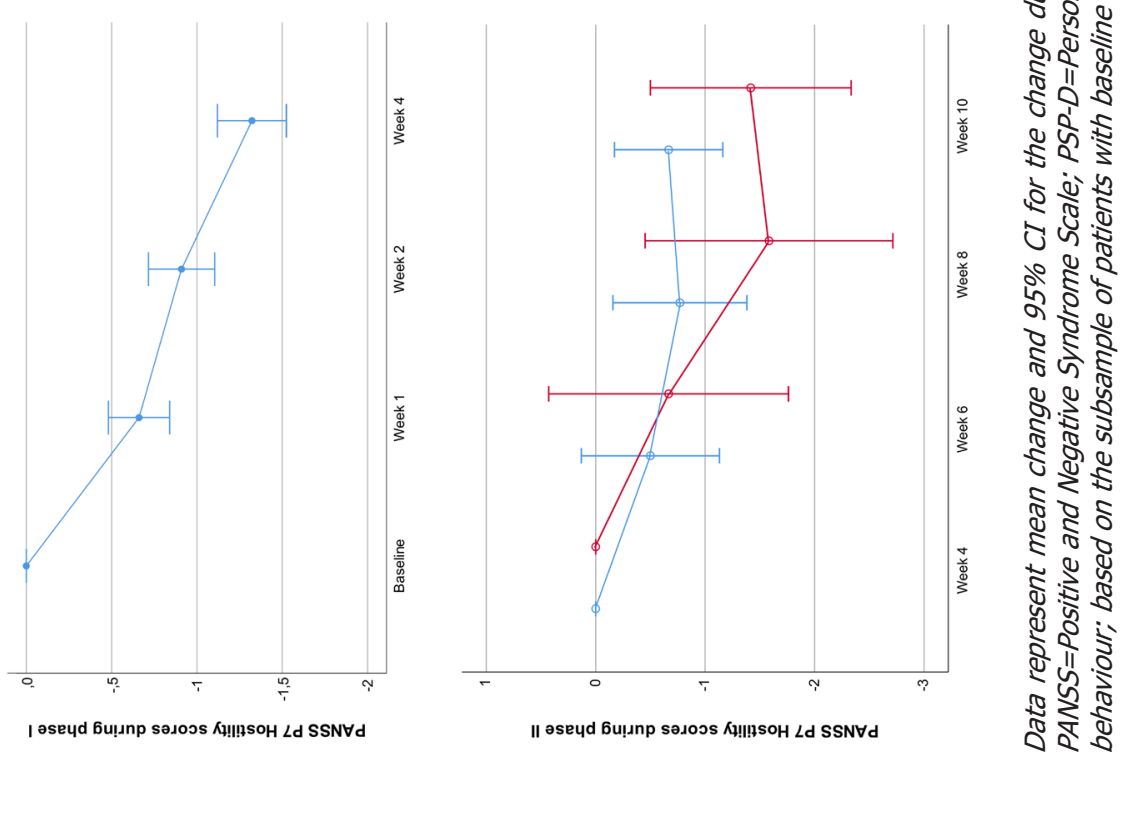


\begin{tabular}{|c|c|c|c|c|c|c|}
\hline & & $\begin{array}{l}\text { Baseline } \\
\text { phase II }\end{array}$ & Week 6 & Week 8 & Week 10 & $\begin{array}{l}\text { Baseline to } \\
\text { week } 10\end{array}$ \\
\hline \multirow[t]{4}{*}{$\begin{array}{l}\text { PANSS P7 } \\
\text { hostility }\end{array}$} & $\begin{array}{l}\text { AMI, } n=16 \\
\text { Mean (SD) }\end{array}$ & $3.1(1.0)$ & $2.6(1.3)$ & $2.4(1.3)$ & $2.5(1.2)$ & \\
\hline & Mean difference (SD) & & $-0.5(1.1)$ & $-0.4(1.1)$ & $0.1(0.8)$ & $-0.7(0.8)$ \\
\hline & $\begin{array}{l}\text { OLA, } n=12 \\
\text { Mean (SD) }\end{array}$ & $3.8(1.1)$ & $3.1(1.8)$ & $2.2(1.5)$ & $2.3(1.6)$ & \\
\hline & Mean difference (SD) & & $-0.7(1.7)$ & $-0.9(1.1)$ & $0.2(1.3)$ & $-1.4(1.4)$ \\
\hline \multirow[t]{4}{*}{ PSP-D } & $\begin{array}{l}\text { AMI, } n=14 \\
\text { Mean (SD) }\end{array}$ & $1.8(1.1)$ & $1.9(1.2)$ & & $1.8(1.3)$ & \\
\hline & Mean difference (SD) & & $0.1(0.8)$ & & $-0.1(0.8)$ & $.0(1.0)$ \\
\hline & $\begin{array}{l}\text { OLA, } n=10 \\
\text { Mean (SD) }\end{array}$ & $3.0(1.5)$ & $2.1(1.3)$ & & $2.2(1.5)$ & \\
\hline & Mean difference (SD) & & $-0.8(1.0)$ & & $0.0(0.5)$ & $-.7(0.8)$ \\
\hline
\end{tabular}

PANSS=Positive and Negative Syndrome Scale; PSP-D=Personal and Social Performance Scale domain D: disturbing and aggressive behaviour; AMI=amisulpride, OLA=olanzapine; data based on the subsample of patients with baseline hostility $>1$.

\section{Discussion}

The prevalence of moderate, severe and extreme hostility (9.4\%) and manifest, marked, severe or very severe disturbing and aggressive behaviour (20.2\%) in this sample of FEP patients is low. As patients participating in a randomized controlled trial (RCT) are considered a selected sample, this may not reflect the total population of FEP patients. We found PANSS P7 hostility and PSP-D disturbing and aggressive behaviour low to moderate but significantly associated with each other and with PANSS items delusions, hallucinatory behaviour, tension, unusual thought content and lack of judgement and insight. The strongest associations, although still moderate, for both hostility and disturbing and aggressive behaviour were with poor impulse control, uncooperativeness and excitement. In the first phase of the trial, amisulpride use was associated with a reduction in hostile and aggressive behaviour and this association was still significant after controlling for baseline PANSS positive symptoms. In the second phase of the trial, we could not demonstrate a difference between continuation of amisulpride and a switch to olanzapine.

The prevalence of hostile and aggressive behaviour is the current sample is lower compared with other studies. In the EUFEST sample, $60.1 \%$ of the patients reported baseline hostility $>1$ compared with $41.5 \%$ patients in the current OPTiMiSE sample. 
The mean hostility score at baseline in the EUFEST sample was $3.2(S D=1.1)$ versus $3.0(S D=1.1)$ in the OPTiMiSE sample. When comparing these results with rates of violent behaviour in FEP, it should be mentioned that a hostility score of 2 is a low threshold to label behaviour as hostile. PANSS hostility score of 2 indicates "minimal; may be at the upper extreme of normal limits" (Kay et al., 1987). Moderate, severe or extreme aggression (i.e. PANSS hostility $>3$ ) was present in only 42 patients $(9.4 \%)$ of the intention-to-treat sample at baseline. This is still lower than meta-analytic data indicating that around 30\% of FEP patients engage in any violence (Large \& Nielssen, 2011; Winsper et al., 2013).

There were differences between PANSS and PSP-D scores as only 127 patients $(28.5 \%)$ scored on both scales. It is expected that patients score on the PSP but not on the PANSS as the PSP is based on behaviour during the past month and PANSS is aimed at behaviour during the past week. However, 32\% of the hostility subsample did not score on the PSP-D at baseline. This could be because the PANSS is more sensitive than the PSP-D and questionable forms of hostility are not included on the latter.

The results from the correlational analysis are comparable with previous work. Combined analyses of the CATIE and EUFEST trials also found hostility associated with positive symptoms and lack of judgement and insight (Volavka et al., 2016). We confirmed the association for impulsivity and hostility with aggressive behaviour in FEP (Moulin et al., 2018a; Moulin et al., 2018b). Additionally, we found excitement and uncooperativeness also associated with aggressive behaviour in a population of FEP. Meta-analytic data from psychotic disorders in general also found excitement associated with violence, but not uncooperativeness (Witt et al., 2013). More recent work found both excitement and uncooperativeness associated with different aggressiveness outcomes, although with differences between these measures (Faay et al., 2020). Compared with the different results considering the link between positive symptoms and violent behaviour in FEP (Brucato et al., 2019; Coid et al., 2013; Langeveld et al., 2014; Large and Nielssen, 2011) and psychotic disorders in general, where the positive symptom subscale is associated with violence (Volavka et al., 2016; Witt et al., 2013), we found hallucinations, delusions and unusual thought content low but significantly associated with hostility and disturbing and aggressive behaviour.

The reduction in hostility scores in phase $\mathrm{I}$ is in line with previous work. The mean reduction in hostility scores after one month for patients on amisulpride in the EUFEST study was around -1.2 (Volavka et al., 2011), which is comparable with our results at week $4(-1.3)$. In both studies, the effect was still significant after controlling for other positive symptoms.

In the second phase of the trial, we could not find a significant difference in effect between amisulpride and olanzapine or a significant reduction over consecutive 
visits. This is likely due to the low sample size and a ceiling effect since the mean scores at baseline phase II are low and there can only be little improvement. In the EUFEST trial, olanzapine was significantly superior to amisulpride in reducing hostility during the first month of treatment $(p<.05)$ (Volavka et al., 2011). The CATIE trial found olanzapine superior to other antipsychotics but did not use amisulpride (Volavka et al., 2014).A recent meta-analysis found that amisulpride and olanzapine reduce symptoms of schizophrenia to a similar degree (Huhn et al., 2019). Although the mechanisms of the effect of antipsychotics on aggression and hostility are still not fully understood, aggression is heterogeneous. Not all aggression from patients with psychotic disorders may be derived from psychosis. Therefore, efficacy on hostility may change among antipsychotics, not only because of the general effect of antipsychotics, but also because of differences in effect on sedation or other symptoms.

The current results have several implications for clinical practice. First, poor impulse control, excitement and uncooperativeness could be more important factors in violent behaviour than positive symptoms. This could help clinicians in observing and treating these kinds of behaviours. Second, these results contribute to the current understanding of the effect of antipsychotics on aggressive behaviour. Amisulpride was an effective agent in the treatment of hostile and aggressive patients and this effect is, at least partly, independent from baseline positive symptoms, but placebo-controlled studies are needed to confirm this effect. Moreover, while other studies are aimed at the long term effect of medications on hostility, the current results are focused on the effect in the first 10 weeks of treatment after the FEP, where patients are at particular risk for adverse behaviour, thus providing insight into the clinical factors and problems in these first weeks of treatment. We believe the current results imply that amisulpride should be considered for FEP patients with hostile or aggressive behaviour during the first weeks of treatment, specifically when olanzapine is contra-indicated because of the risk for weight-gain.

\section{Limitations}

The current study has several limitations. First, the OPTiMiSE trial was not designed for an analysis on hostile or aggressive behaviour. There will probably be some selection bias as patients were able to participate in a medication trial and sign an Informed Consent. Coercive treatment was an exclusion criterion and hostile patients may be more inclined to refuse study participation. The included patients are therefore likely to display less aggressive behaviour compared with FEP patients that are admitted to a hospital on an involuntary basis. Specifically in phase II, the sample size of the hostility subsample is low and this may have hampered the comparison between olanzapine and amisulpride. Second, by using two measures, we provide an indication of the level of aggressive and hostile behaviour but we do not have information on aggressive 
incidents. Incorporation of specific scales that measure aggression, such as incident report forms, is needed for a more objective measure of aggressive behaviour. Last, the PSP was not used according to its guidelines. The PSP is aimed at the behaviour in the past month but was assessed multiple times within one month, thus possibly losing subtle changes in patients' behaviour. Also, patients were selected based on their PANSS P7 hostility score and not on their PSP-D score.

\section{Future research}

Future research should address differential effects of different antipsychotic medication on hostility in FEP, in particular a comparison between olanzapine and amisulpride may be interesting in a sample of patients with more severe hostility scores. We hypothesize that amisulpride and olanzapine are equally effective against hostility during the first weeks of treatment and that a switch could be effective after a non-response for several weeks of treatment. This should ideally be studied with combined outcome measures such as PANSS P7 hostility and data from incidents reports. Moreover, research should focus on the effect of different dosages antipsychotics and the use of concomitant benzodiazepines on aggressive behaviour in FEP. For clinicians, these are interventions frequently used in order to prevent or treat aggressive behaviour. Although there is some evidence that higher dosages of antipsychotics are more effective (Faay et al., 2018; Fazel et al., 2014), many questions about these effects remain. For the effect of benzodiazepines on aggression or agitation in patients with psychosis there is little evidence (Baranchik et al., 2019; Zaman et al., 2018) and the decreased inhibition benzodiazepines cause might also increase impulsivity and dangerous behaviour (Guina and Merrill, 2018). As it is difficult to detangle cause from consequence, this should ideally be studied in a trial with fixed dosages and regulated benzodiazepine use.

\section{Conclusion}

The prevalence of moderate to severe hostility (9.4\%) and disturbing and aggressive behaviour $(20.2 \%)$ in this sample of FEP is low. We found a significant reduction in PANSS hostility and PSP-D scores after 4 weeks of amisulpride which remained significant after controlling for baseline positive symptoms, thus indicating that amisulpride could be an effective antipsychotic choice in the treatment of FEP patients who express hostile or aggressive behaviour. Future research is needed to compare the effects of amisulpride and olanzapine on hostility in FEP during the first weeks of treatment. 
Clinical risk factors such as delusions, hallucinatory behaviour, tension, unusual thought content and lack of judgement and insight could be important for risk assessment and treatment planning. But the most meaningful clinical risk factors in detecting and treating hostile and aggressive behaviour, are poor impulse control, uncooperativeness and excitement as these symptoms could be observed from patients' behaviour and thereby help clinicians in detecting and treating hostile and violent behaviour in FEP. 



\section{CHAPTER 6}

\section{Efficacy of typical and atypical antipsychotic medication on hostility in patients with psychosis- spectrum disorders: a review and meta-analysis}

Margo Faay

Pál Czobor

Iris Sommer

Neuropsychopharmacology (2018), 43(12), 2340-2349 
As violence against self and others is an important outcome in the treatment of patients with psychosis-spectrum disorders and hostility is an important indicator for violence, we set out to evaluate the effects of different types of antipsychotic agents in reducing hostility. We performed a systematic literature search which provided 18 suitable randomized studies comparing typical to atypical antipsychotics for at least 4 weeks in patients with psychotic disorders. Results showed a small (0.26) but significant effect for atypical as compared to typical antipsychotics, with high heterogeneity, even though the mean dose of typical antipsychotics was higher. This effect size remained similar when separately analysing sponsored and non-sponsored studies. When differentiating between high and low-dose studies, the high-dose group showed a significant difference between typical and atypical antipsychotics whereas the low-dose group did not. An analysis comparing clozapine to typical antipsychotics showed a moderate effect size (0.415), with low heterogeneity. These results are important for clinicians to help their shared decision making with patients when choosing maintenance treatment, as next to efficacy for psychosis and tolerability, safety for the patient and their environment is an important outcome.

\section{ABSTRACT}




\section{Introduction}

Violence is an important outcome measure in patients who suffer from psychosisspectrum disorders. A large meta-analysis including 45,533 patients with psychosisspectrum disorders or bipolar disorder found that $18.5 \%$ had committed a verbal aggressive or physical violent act (Witt et al., 2013). An earlier meta-analysis including 18432 patients with psychosis-spectrum disorders found that $9.9 \%$ of the patients committed at least one act of interpersonal violence or violent criminality including threats (Fazel et al., 2009). This meta-analysis also found an increased risk for homicide in individuals with schizophrenia of $0.3 \%$ versus $0.02 \%$ in the general population (Fazel et al., 2009). A third meta-analysis including only patients with a first- episode psychosis found that $34.5 \%$ had shown physical violence with almost half of these (16.6\%) having committed serious violence (resulting in injury/ using a weapon/ sexual assault) (Large \& Nielssen, 2011). Factors such as the severity of positive symptoms, comorbid substance abuse, economic deprivation, childhood conduct disorder and poor treatment adherence are well known prospective predictors, while negative symptoms predict lower rates of violence (Hodgins \& Riaz, 2011; Volavka \& Citrome, 2011; Witt et al., 2013).

Violence has several negative consequences for patients themselves and for the general public. Although 95 to $99 \%$ of violent incidents in society is not caused by individuals with psychosis-spectrum disorders, a certain public fear still exists (Taylor, 2008).Violence of patients with psychosis-spectrum disorders contributes to the stigmatization for those suffering from psychotic illness, affecting also the large majority without any history of violence. The consequences of violence for individual patients include legal involvement, prolonged hospitalization, individual stigmatization but also victimization (Hodgins, 2008).

Previous reports suggest that in the acute phase of psychosis, antipsychotic medication prevents or reduces aggressive behaviour (Hodgins \& Riaz, 2011; Large $\&$ Nielssen, 2011). There is the suggestion for superiority of clozapine in reducing violence, specifically in treatment-resistant psychotic disorders (Frogley et al., 2012; Glazer \& Dickson, 1998; Spivak, Mester, Wittenberg, Maman, \& Weizman, 1997). However, most of these studies are in retrospect. It has also been suggested that atypical antipsychotics, which on average are more potent 5-HT antagonists, could have a better anti-aggressive effect than more specific D2 blockading drugs (Aleman \& Kahn, 2001; Buckley, 1999). Yet, there is no consistent evidence to make a rational decision as to which type of antipsychotic may be most effective in reducing aggression and even the superiority of clozapine is not well documented. The aim of the current review study is to provide evidence to support a rational decision on which type of antipsychotic medication most effectively reduces hostility and thereby possibly 
affecting violence in patients with psychosis-spectrum disorders.

Violence and aggression can be assessed in many different ways. Unfortunately, very few studies have investigated the influence of different antipsychotic drugs on actual rates of violent incidence or added specific questionnaires inquiring about such incidents. However, many randomized controlled trials provide data on the hostility item of the Positive and Negative Symptom Scale (PANSS; (Kay et al., 1987)) or the Brief Psychiatric Rating Scale (BPRS; (Overall \& Gorham, 1962)). This item is often used to give an indication of the aggressiveness of a patient. There is a close association between the hostility item and aggression since some of the descriptions of the hostility item include verbal aggression and physical violence (Volavka \& Citrome, 2011). Indeed, a significant correlation $(r=.77, p<0.01)$ between the hostility item of the PANSS and the Modified Overt Aggression Scale (MOAS) has been found (Knezevic et al., 2017) as well as a significant association $(O R=1.5,95 \% C I$ 1.0-2.1) between PANSS hostility scores and verbal aggressive or physical violent incidents (Witt et al., 2013).

Therefore, we assessed the efficacy of various antipsychotic drugs on this PANSS/BPRS item, focusing on the comparison between typical and atypical antipsychotics, in studies of at least 4 weeks in duration.

\section{Materials and Methods}

\section{Search}

A meta-analysis was conducted according to the PRISMA statement (Moher, Liberati, Tetzlaff, \& Altman, 2009). An electronic search was conducted using PubMed, Cochrane, Embase and PsychInfo. The following Mesh search terms were used both alone and in combinations: "violence," "aggression," "hostility," "schizophrenia," "psychotic disorders," "antipsychotic agents" and the generic names of 25 commonly used antipsychotics. There was no limitation on year or language. The reference lists of the obtained studies were checked for cross references.

\section{Inclusion}

The following inclusion criteria were used:

- Method: randomized studies, blinded or open label.

- Population: patients diagnosed with a psychosis-spectrum disorder (schizophrenia, schizoaffective disorder or psychotic disorder not otherwise specified) according 
to the Diagnostic and Statistical Manual of Mental Disorders (DSM-III, DSMIII-R, DSM-IV, DSM-IV-TR or DSM-V or International Classification of Diseases (ICD 9 or 10). Both in and out patients were included but not patients under current treatment in forensic facilities.

- Medication: studies compared at least one typical to one atypical antipsychotic.

- Treatment duration: studies testing the antipsychotic for four weeks or more.

- Outcome measure: studies reporting the hostility item of the PANSS or BPRS, the PANSS hostility/excitement factor score, the BPRS hostile/suspiciousness cluster or the Behavioural Agitation Score (BAS) derived from the BPRS.

- Data: studies reporting sufficient information to compute common effect size statistics (means and $S D s$, exact $p, t$, or $z$ values) or corresponding authors could supply these data upon request.

We excluded case studies, cross sectional studies and studies using a mixed sample of psychotic disorders and other diagnosis. The search included studies aimed specifically on hostility (i.e., studies that reported on hostility or violence in title, abstract or keywords). After the initial search, we used cross references from the studies we found and other reviews.

\section{Outcome measures}

On the PANSS, the hostility item is defined as "verbal and nonverbal expressions of anger and resentment, including sarcasm, passive-aggressive behaviour, verbal abuse and assaultiveness." The score is based on interpersonal behaviour during the interview and reports by primary care workers or family. It is, like the other PANSS items, scored from 1-7. A score of 1 indicates absent hostility whereas a score of 7 indicates marked anger, resulting in physical assault towards others. The hostility item of the BPRS is similarly scored on a scale 1-7 and is defined as "animosity, contempt, belligerence, threats, arguments, tantrums, property destruction, fights, and any other expression of hostile attitudes or actions. Do not infer hostility from neurotic defences, anxiety or somatic complaints. Do not include incidents of appropriate anger or obvious selfdefence." Although both instruments use a scale of 1-7 and assess the same construct, there are differences. Where the PANSS instructions include anchors for each score of 1 through 7, the original BPRS does not include anchors for individual scores but only a description of the hostility item.

For both the PANSS and the BPRS, clusters have been made to assess and differentiate domains in psychopathology more accurately. The PANSS five factor model is developed by Lindenmayer et al. (1994), and sometimes called "uncontrolled hostility/excitement factor." This cluster consists of four items: P4 excitement, G14 poor impulse control, P7 hostility and G4 tension. The BPRS factor is named 
"hostility/suspiciousness" and consists of the items hostility, suspiciousness and uncooperativeness (Overall \& Gorham, 1962). For the BPRS, a second cluster is sometimes used: the Behavioral Agitation Score (BAS), also called the BPRS agitation score. The BAS contains the items: hostility, anxiety, tension, uncooperativeness and excitement (Chengappa, Goldstein, Greenwood, John, \& Levine, 2003). The primary outcome measure was the mean change in the hostility item of the PANSS or BPRS, the PANSS hostility/excitement factor score, the BPRS hostile/suspiciousness cluster or BAS for typical versus atypical antipsychotics. If both the hostility item and the factor or cluster score were reported, the hostility item was preferred.

Patient data of last observation carried forward (LOCF) were used for analysis. If studies did not provide the (exact) data, the authors were contacted requesting the data.

\section{Comparisons}

Primary analysis is to compare atypical to typical antipsychotics.

To check for a possible dose-effect, the studies were divided into two groups: one with the typical and atypical antipsychotics dosed below $500 \mathrm{mg}$ chlorpromazine equivalent and one with dosages above $500 \mathrm{mg}$ chlorpromazine equivalent. Moreover, since clozapine is suggested to be particularly effective in reducing hostility (Glazer \& Dickson, 1998; Spivak et al., 1997), a separate analysis was performed including only this agent in comparison to typical antipsychotic medication. Also, the effect sizes of the sponsored versus non-sponsored studies as well as the open label versus double blind studies were calculated.

\section{Analysis}

Two reviewers independently extracted data. Disagreements were resolved by consensus. Hedges' $g$ was used to quantify effect sizes for the mean difference (typical vs atypical) of the change score (end of treatment vs baseline). These change scores were preferred instead of pre-treatment and post-treatment scores in order to avoid overestimation of the true effect because of the pre-post treatment correlation (Dunlap, Cortina,Vaslow, $\&$ Burke, 1996). If no change score was reported, pre- and post-treatment means and standard deviations (SDs) or exact $\mathrm{F}, t$, or $p$ values were used.The pre post correlation was posited as 0.5 in terms of Pearson correlations. If a comparison was reported to be significant, but no exact $\mathrm{p}$-value was reported, the reported $\mathrm{p}$-value threshold was used. For example, if only $p<0.01$ was given; $p=0.01$ was used. The effect was considered positive when the atypical antipsychotic had a better effect on hostility than the typical antipsychotic. 
When studies had more than one arm of atypical antipsychotics (e.g., two or more atypical antipsychotics or different dose of the same antipsychotic), the data of the typical antipsychotics arm was used multiple times as a control. Since this could cause an under- or overestimation of the effect of the typical antipsychotic in terms of significance, the $\mathrm{N}$ of the typical group was divided by the number of atypical arms in the study.

A random effects model was used because of the differences between studies in participants, treatment duration and size (Borenstein, Hedges, Higgings, \& Rothstein, 2009). To measure the heterogeneity the $I^{2}$ was calculated. Heterogeneity of $<25 \%$ is considered low, $>50 \%$ is moderate and a value of $>75 \%$ indicates a high variance (Borenstein, Higgins, Hedges, \& Rothstein, 2017). High heterogeneity poses a limitation to a reliable interpretation of the results. However, it had been discussed that $I^{2}$ does not indicate how much the effect size varies (Borenstein et al., 2017). A sensitivity analysis was undertaken by repeating the analysis excluding outlier studies. All calculations were executed using Comprehensive Meta-Analysis Version 2.0, Biostat (Borenstein, Hedges, Higgings, \& Rothstein, 2005).

The chlorpromazine equivalent levels were calculated according to the study of Gardner et al. (2010). The reported fixed or mean dose of the antipsychotics in the included studies were used. These equivalents were used to divide the studies into groups using high (>500mg chlorpromazine equivalent) and low $(<500 \mathrm{mg}$ chlorpromazine equivalent) dose antipsychotics. When studies reported the fixed or mean dose at baseline as well as during the study and at endpoint, the dose at endpoint was used. When studies did not report a mean or fixed dosed but a range, the minimum dose of the range should be $>500 \mathrm{mg}$ for the arm to be included in the high dose group.

\section{Results}

The literature search resulted in 1395 studies which were screened for inclusion (Figure 1). After this first screen, 65 articles were assed for eligibility. 42 studies were excluded for specific reasons, for example studies comparing two atypical antipsychotic drugs, non-randomized studies or studies using a treatment period shorter than one month. A total of 18 randomized controlled trials, providing specific information on hostility comparing an atypical antipsychotic to a typical drug for at least four weeks could be included in the meta-analysis (Table 1). Data from 6799 patients were used. From these patients, 4969 were assigned to an atypical antipsychotic and 1830 to a typical antipsychotic. Of these 18 studies, 14 studies used haloperidol as a typical comparator, two used chlorpromazine and one study used perphenazine. Two studies 
used four different atypical antipsychotics and one study used three. Also, three studies differentiated the groups according to different atypical antipsychotic dosages. One study used two dose groups, one study four and one study used five different dose groups. This resulted in a total of 34 subgroups used for the analysis.

As for the atypical antipsychotic drugs, seven studies investigated risperidone, five studies clozapine, four studies olanzapine and quetiapine, ziprasidone and amisulpride were all used in three studies. Two studies were placebo-controlled but the placebo arms are not included in this meta-analysis. With the exception of two studies, all studies were double-blind. A large proportion of the studies (14 studies; 5676 (83\%) patients) were sponsored by pharmaceutical companies.

Figure 1: PRISMA flow diagram of the literature search

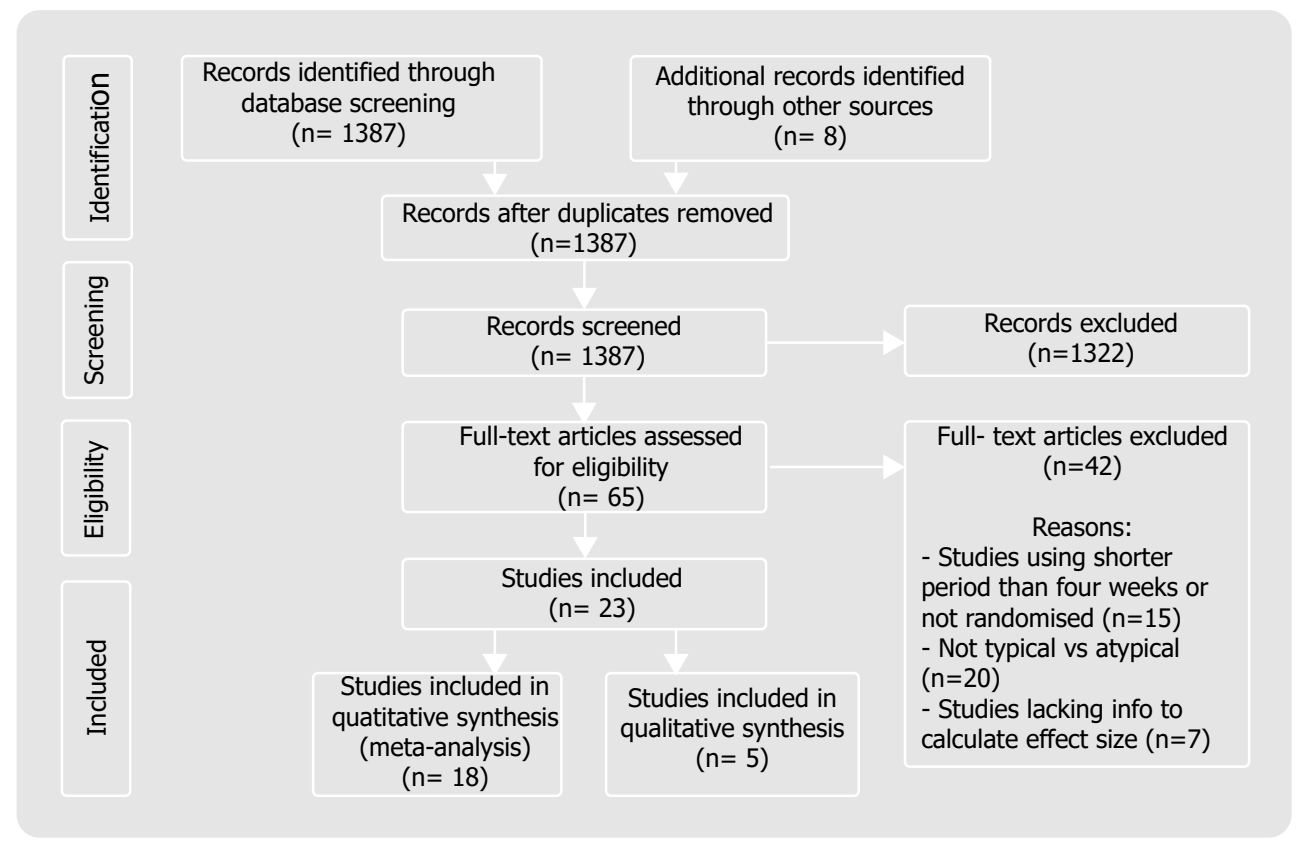




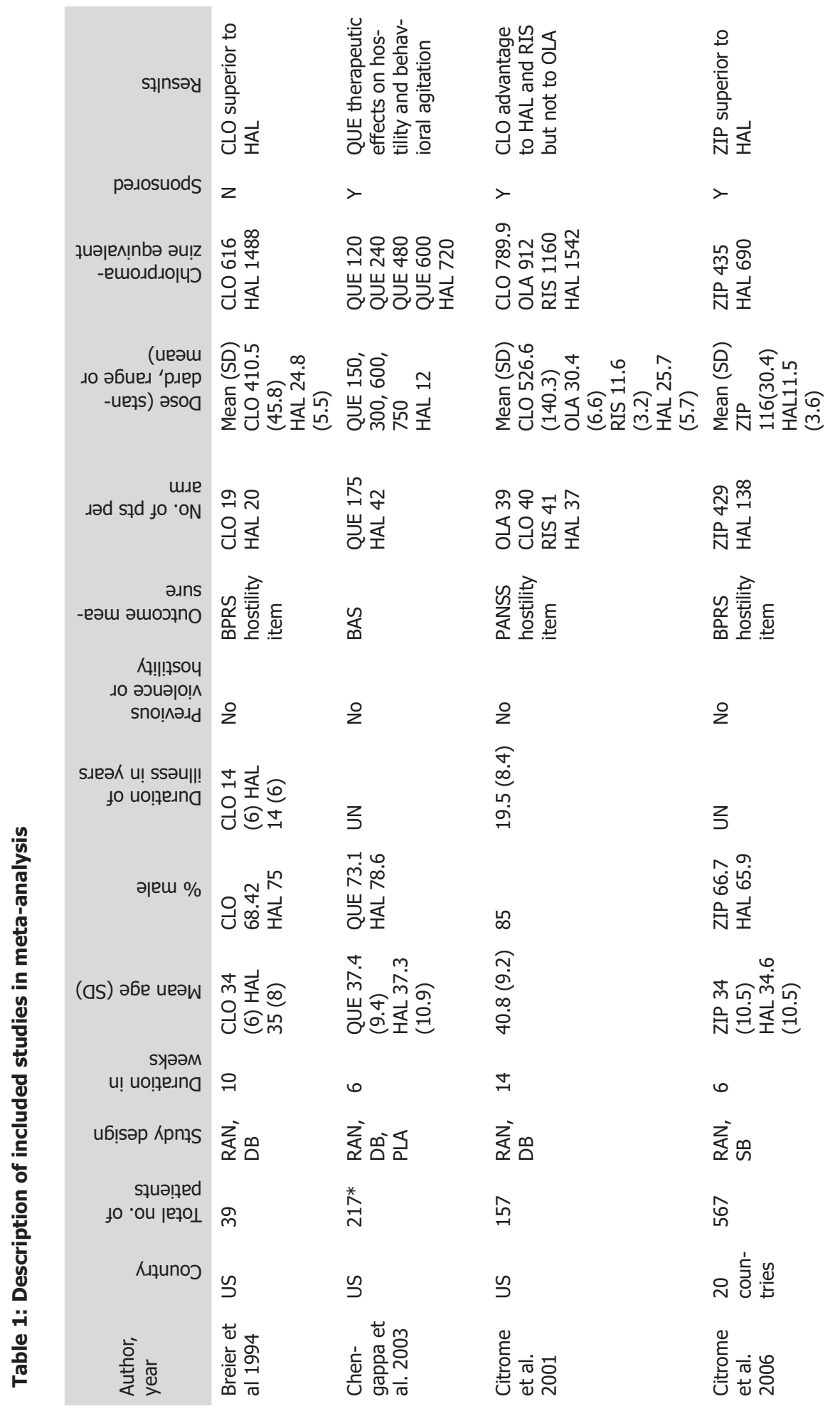




\begin{tabular}{|c|c|c|c|c|c|c|}
\hline słpnsəy & 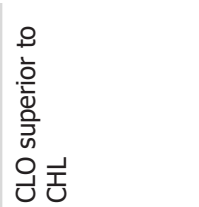 & 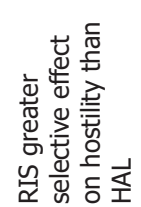 & 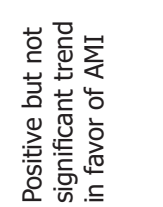 & 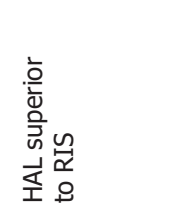 & 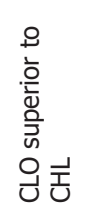 & 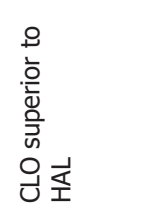 \\
\hline pəגosuods & z & $\succ$ & $z$ & $\succ$ & $\succ$ & $>$ \\
\hline 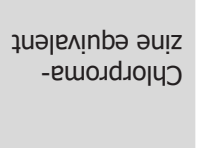 & 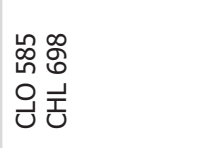 & 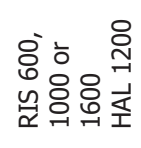 & 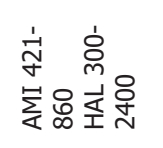 & 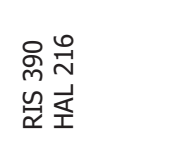 & 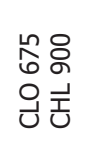 & 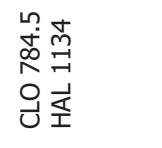 \\
\hline 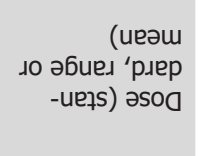 & 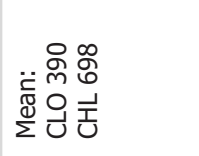 & 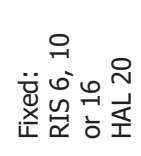 & 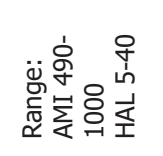 & 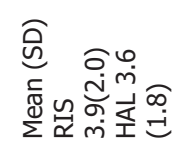 & 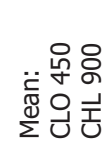 & 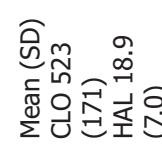 \\
\hline $\begin{array}{r}\text { mue } \\
\text { tad słd to . ON }\end{array}$ & 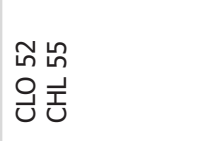 & 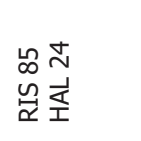 & 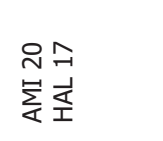 & 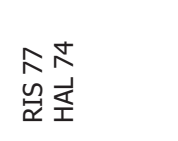 & 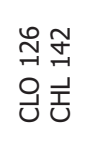 & 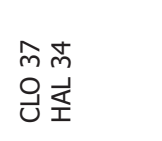 \\
\hline $\begin{array}{r}\text { əגns } \\
\text {-еәш әшоэłnо }\end{array}$ & 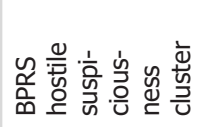 & 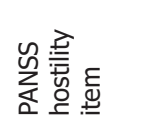 & 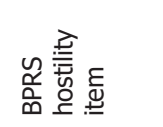 & 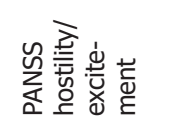 & 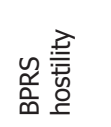 & 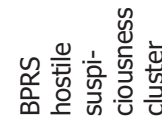 \\
\hline 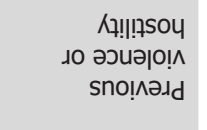 & $\stackrel{0}{2}$ & 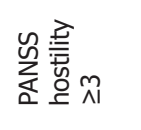 & $\stackrel{0}{2}$ & 2 & 2 & 2 \\
\hline 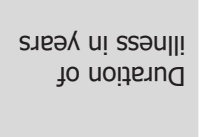 & 3 & 3 & 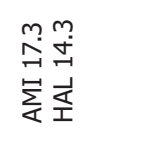 & ろ & z & ろ \\
\hline әәеш \% & $\begin{array}{l}\text { ๙ิ } \\
\text { రิ }\end{array}$ & 3 & $\begin{array}{l}\vec{\infty} \infty \\
\sum_{<}^{\infty} \frac{1}{\mid}\end{array}$ & 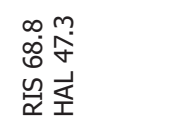 & 3 & 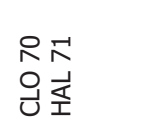 \\
\hline (as) әбе ueəw & 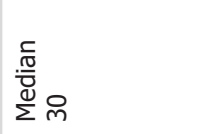 & 3 & 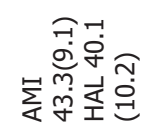 & 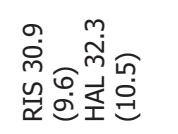 & 3 & 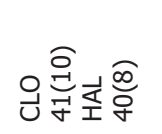 \\
\hline $\begin{array}{r}\text { sуәәмм } \\
\text { u! uo!̣eגna }\end{array}$ & $\infty$ & $a$ & 0 & กิ & 0 & న \\
\hline 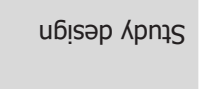 & ऐo & 觓京 & 京回 & 交品 & 京品 & $\sum_{\alpha}$ \\
\hline $\begin{array}{r}\text { squạ̈ed } \\
\text { to :ou |ezol }\end{array}$ & م્વ & 蒙 & $\hat{m}$ & $\overrightarrow{\stackrel{n}{n}}$ & $\stackrel{\infty}{N}$ & $\vec{r}$ \\
\hline kıұunos & 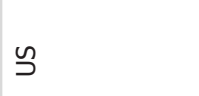 & 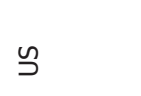 & 总 & 獣 & 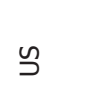 & 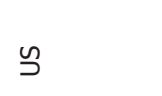 \\
\hline $\begin{array}{l}\text { 蒿 } \\
\text { 妾京 }\end{array}$ & 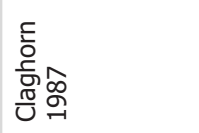 & 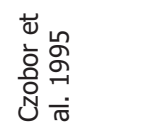 & 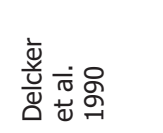 & 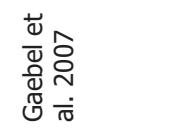 & 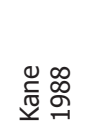 & 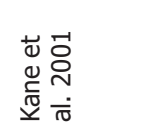 \\
\hline
\end{tabular}




\begin{tabular}{|c|c|c|c|c|c|c|}
\hline sł|nsəy & 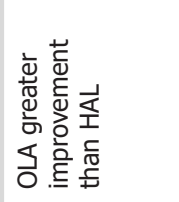 & 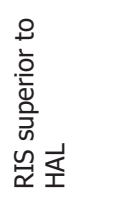 & 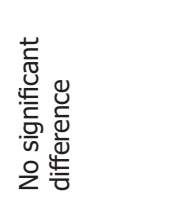 & 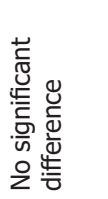 & 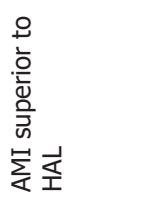 & \\
\hline pəıоsuods & $>$ & $>$ & $>$ & $>$ & $>$ & $>$ \\
\hline 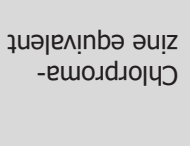 & 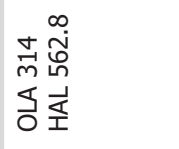 & 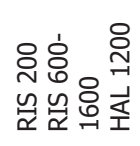 & 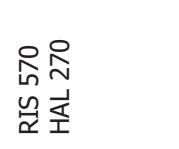 & 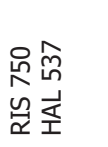 & 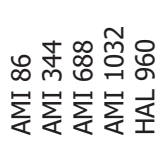 & 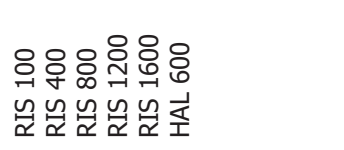 \\
\hline 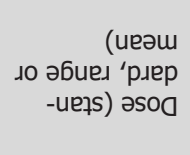 & 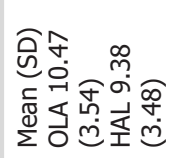 & 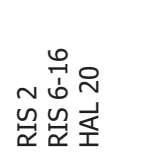 & 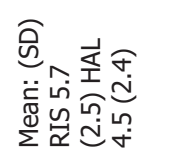 & 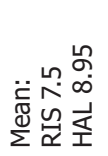 & 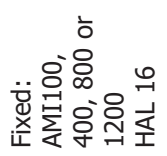 & 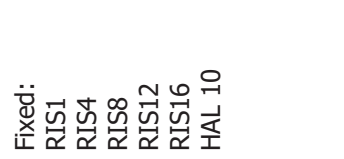 \\
\hline $\begin{array}{r}\text { mue } \\
\text { təd słd to "on }\end{array}$ & 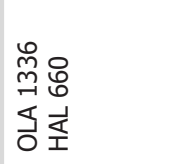 & 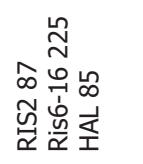 & 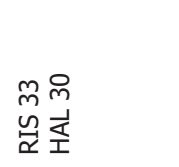 & 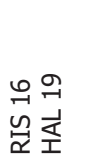 & 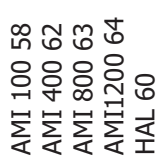 & 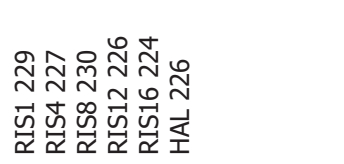 \\
\hline $\begin{array}{r}\text { วגns } \\
\text {-еәш әшоวฺฺno }\end{array}$ & 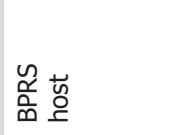 & 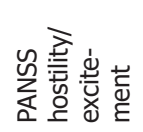 & 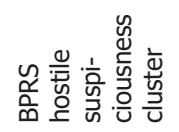 & 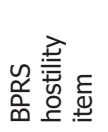 & 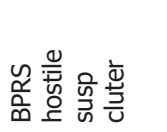 & 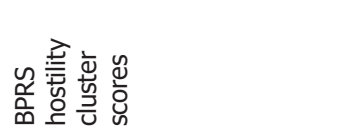 \\
\hline 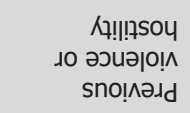 & 운 & 은 & $\frac{0}{2}$ & 운 & 을 & 을 \\
\hline $\begin{array}{l}\text { sıeəК u! ssəu|!! } \\
\text { fo uo!̣eına }\end{array}$ & 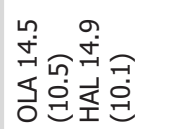 & z & ろ & ろ & ろ & z \\
\hline әреш \% & $\begin{array}{l}\text { 웜 } \\
\text { 它完 }\end{array}$ & m & 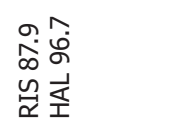 & $\hat{\stackrel{o}{\sigma}}$ & $\widetilde{ช}$ & 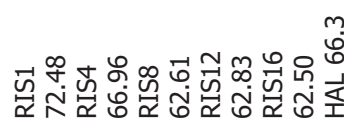 \\
\hline (as) әбе uеәw & 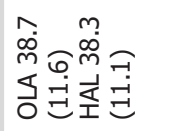 & 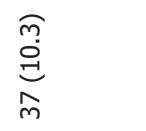 & 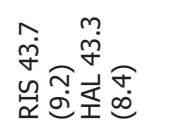 & में & 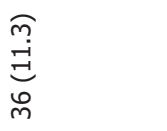 & 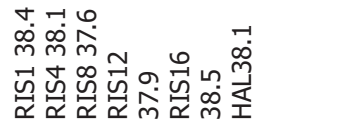 \\
\hline $\begin{array}{r}\text { ऽуәәәм } \\
\text { u! uo!̣e.มng }\end{array}$ & 0 & $\infty$ & 옴 & $\infty$ & $\theta$ & $\infty$ \\
\hline u6!səp Кpnłs & 衣吕 & 京品 & 衣品 & 市品 & 市 & 市 \\
\hline $\begin{array}{r}\text { squarped } \\
\text { to :ou |ezol }\end{array}$ & ஓ요 & 今ે & $\tilde{6}$ & $\stackrel{m}{m}$ & 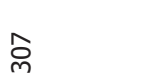 & $\underset{\mathrm{O}}{\mathrm{m}}$ \\
\hline Kıиunos & 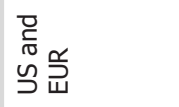 & 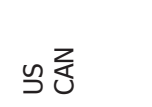 & 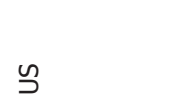 & $\begin{array}{l}\text { 吕 } \\
\text { ㅁ }\end{array}$ & 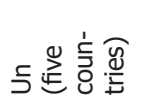 & 녹 \\
\hline 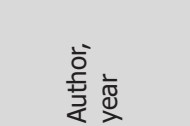 & 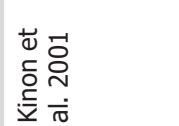 & $\begin{array}{l}\frac{1}{\overline{0}} \\
\frac{1}{\pi} \\
\sum\end{array}$ & 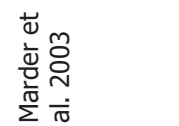 & 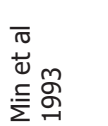 & 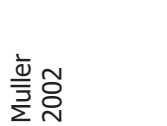 & 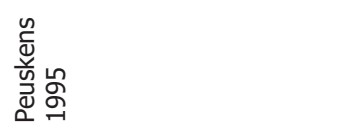 \\
\hline
\end{tabular}




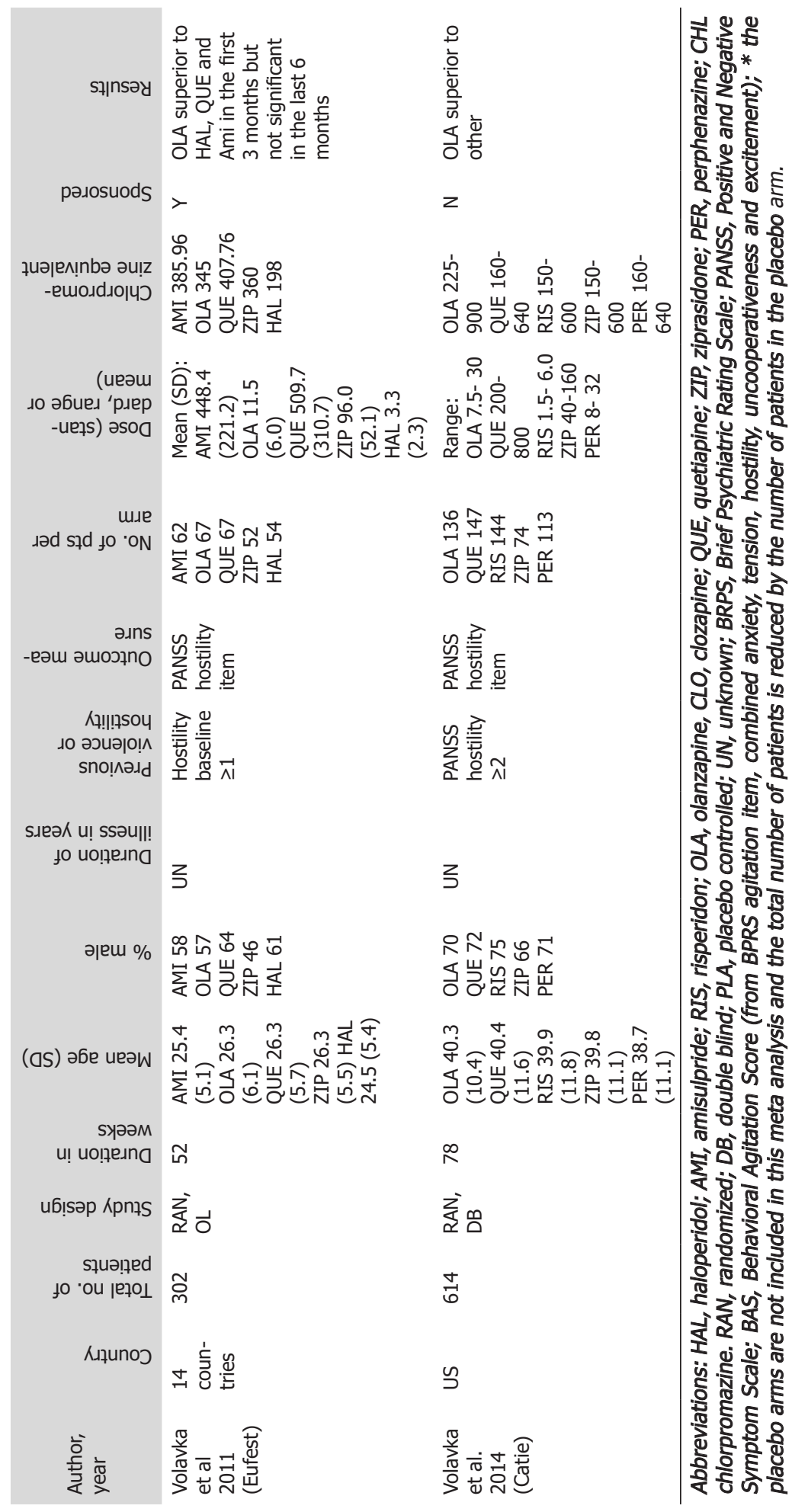




\section{Primary outcome measure: hostility scores}

Antipsychotics were superior in reducing hostility compared to the typical antipsychotics with a small effect size, as shown in Figure 2 (18 studies, $N=6799$, Hedges' $g=0.260, p=0.025)$. Heterogeneity, however, was high $\left(I^{2}=92.65\right)$. The study of Min et al, (Min, Rhee, Kim, \& Kang, 1993) was considered an outlier (see Figure 2). Excluding this study, the effect size remained similar and heterogeneity remained high (17 studies, Hedges' $g=0.273, p=0.020, I^{2}=92.84$ ). The study of Peuskens (1995) included a large group, and for a sensitivity analysis, we repeated the meta-analysis without this study. The effect size remained similar, with increased significance and decreased heterogeneity (17 studies, Hedges' $g=.212, p=.000, I^{2}=44.36$ ).

\section{Differences between high and low dosage on effect size}

The threshold for the high and low dosed groups was set at $500 \mathrm{mg}$ chlorpromazine equivalent. 19 of the subgroups had a dose below $500 \mathrm{mg}$ and 15 subgroups above the $500 \mathrm{mg}$ threshold.

The high dose subgroups showed a significantly superior effect of the atypical antipsychotics in reducing hostility compared with typical antipsychotics (Figure 3; Hedges' $g=.567, p=.001$ ) but this was not found in the analysis of the low-dose groups (Hedges' $g=.023, p=.871$ ). The heterogeneity for the high dosed arms is somewhat lower $\left(I^{2}=82.79\right)$ than the low dose groups $\left(I^{2}=93.39\right)$.

\section{Open label vs double blind studies}

Two studies were open label (Citrome et al., 2006; Volavka et al., 2011). These open label studies had a lower effect size compared with the double blind studies (Figure 4; open label studies Hedges' $g=.136 p=.668$; double blind studies Hedges' $g=.280$, $p=.031)$.

\section{Sponsored vs non-sponsored studies}

For both the non-sponsored as well as the sponsored studies, the atypical antipsychotics were superior in reducing hostility compared to typical antipsychotics (Figure 5; nonsponsored studies Hedges' $g=.248 p=.340$; sponsored studies Hedges' $g=.262 p=.047$ ). Significance was lost for the non-sponsored studies because of reduced statistical power. While mean weighted effect sizes of sponsored and non-sponsored studies were similar, heterogeneity was much higher in the sponsored group $\left(I^{2}=94.12\right)$ compared to the non-sponsored group $\left(I^{2}=12.65\right)$. 


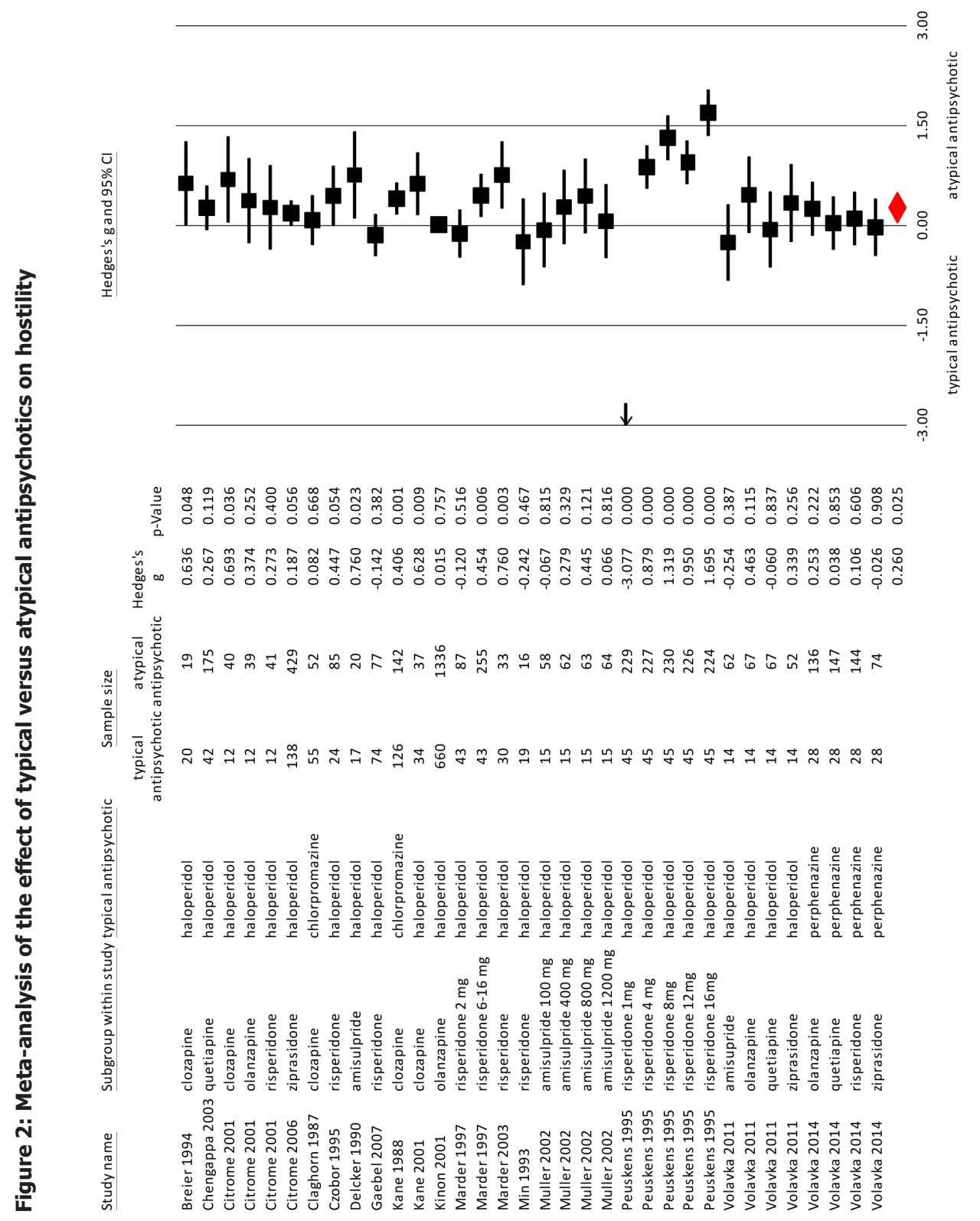




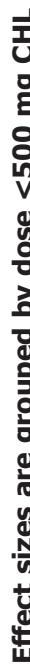

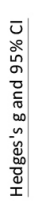

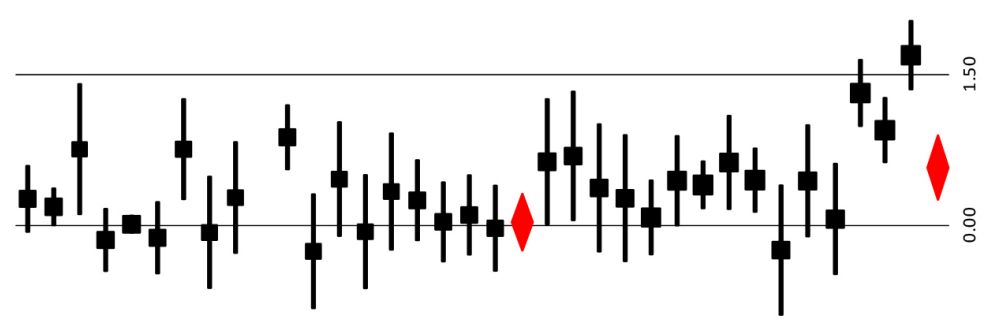

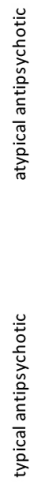

$\downarrow$

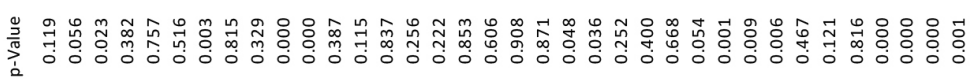

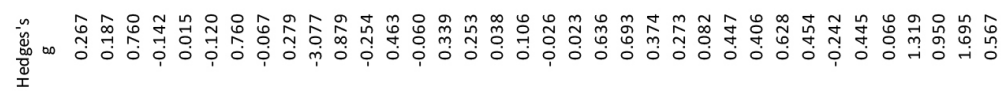

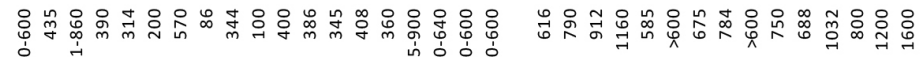 సें

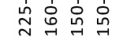

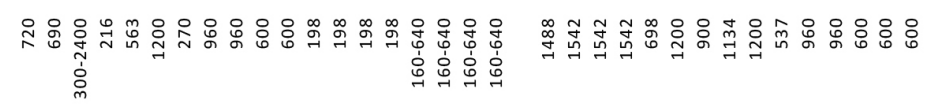

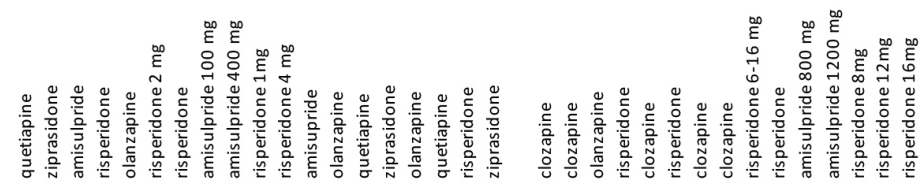



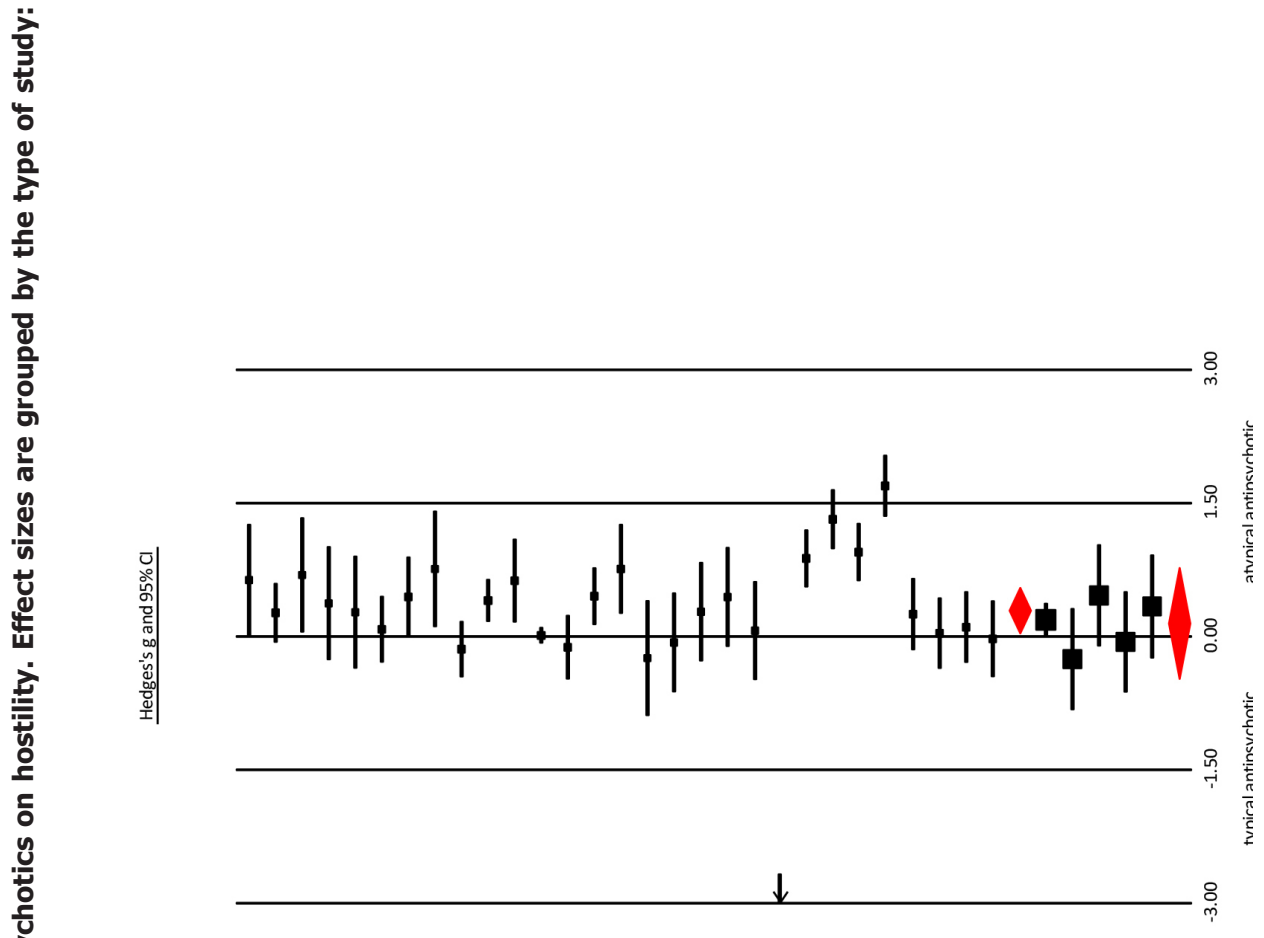

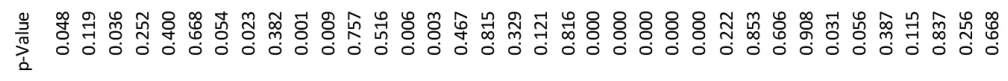

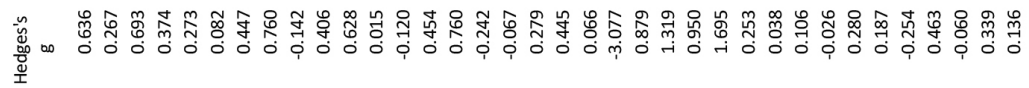
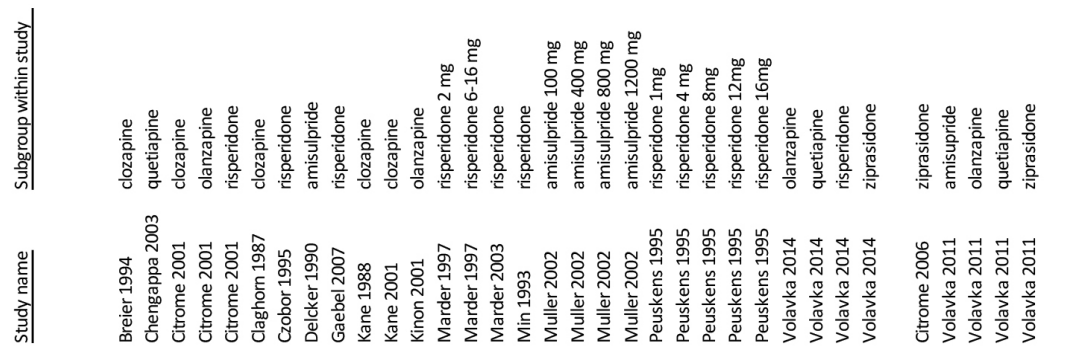

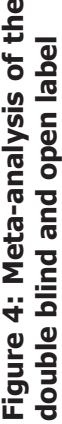

믈
흠
$\frac{0}{0 !}$
$\frac{0}{0}$

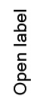



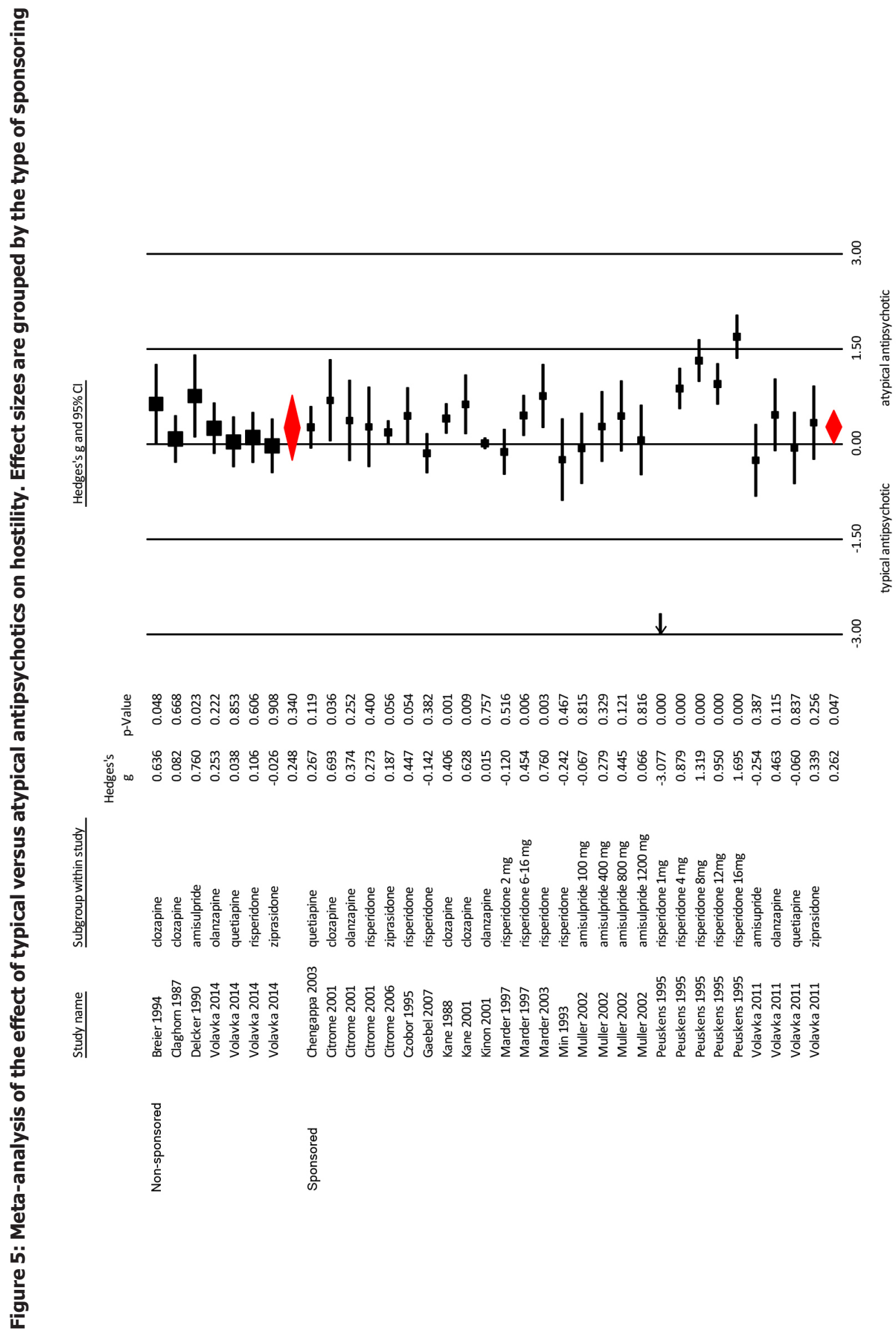

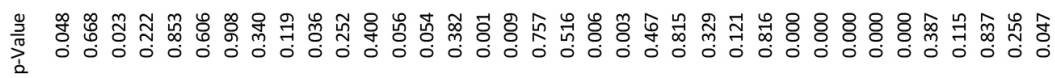

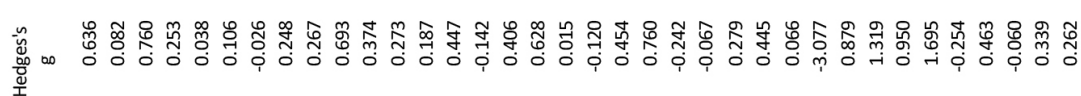

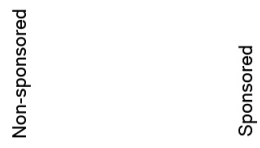




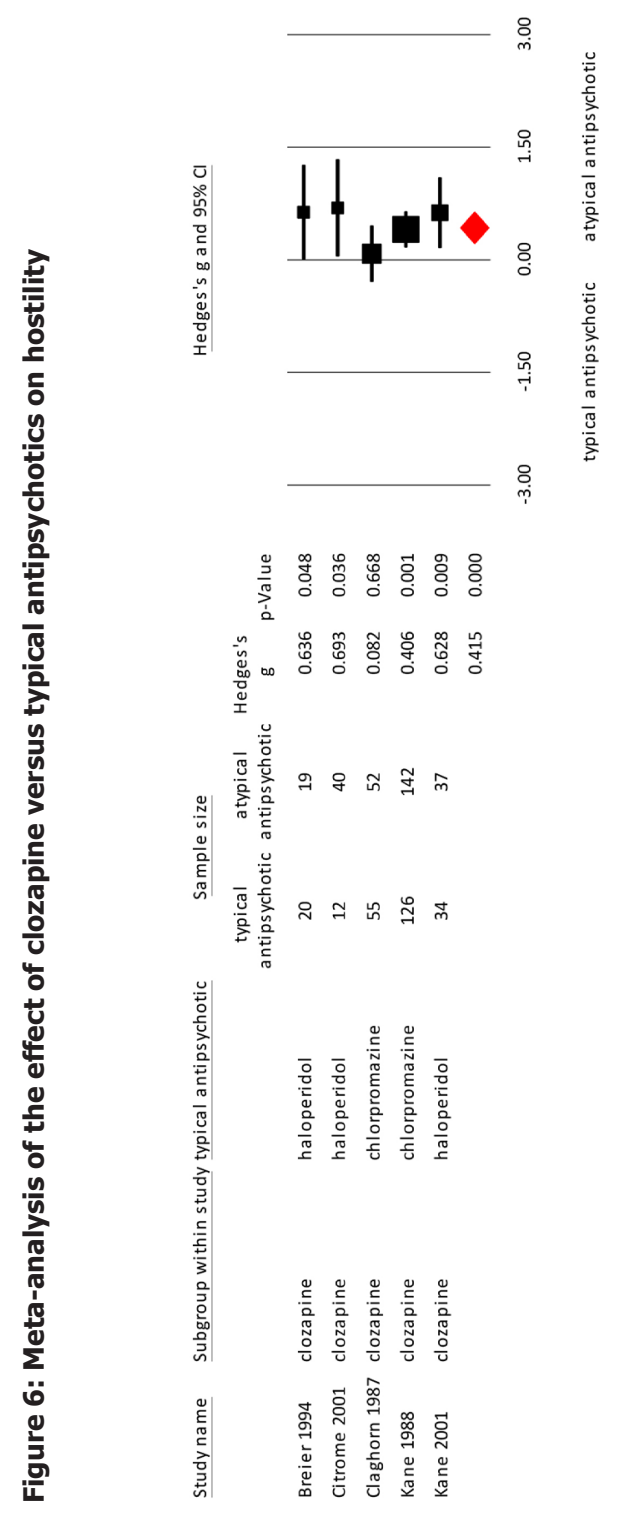




\section{Clozapine}

Five studies compared clozapine to typical antipsychotics, of which three used haloperidol and two studies chlorpromazine as a comparator. Clozapine was significantly superior in reducing hostility compared to typical antipsychotics with a moderate effect size (Figure 6; clozapine $\mathrm{N}=290$; typical antipsychotic (haloperidol or chlorpromazine) $\mathrm{N}=247$; Hedges' $g=.415, p=.0000$; Table 2). Heterogeneity was low $\left(I^{2}=19.16\right)$.

\section{Discussion}

This study found that atypical antipsychotics are marginally more effective in reducing hostility compared to typical antipsychotics, with a small effect size. Heterogeneity, however, was high and the majority of studies were sponsored. Nonetheless, the non-sponsored studies also found atypical antipsychotics to be superior in reducing hostility with a similar effect size. Only two open label studies were included which had a lower effect size than the double blind studies. Clozapine was significantly better in treating hostility than typical antipsychotic drugs, with a moderate effect size and low heterogeneity. In general, typical drugs were dosed higher than atypical drugs. However, with the studies divided into high and low-dose groups, the high dosed atypical antipsychotics show an even greater and significant effect size. It should be noted that the superiority of the high dose atypical antipsychotics could be influenced by the clozapine studies, since these studies are all included in the high dose group.

The results of this meta-analysis largely confirm previous studies (Krakowski, Czobor, Citrome, Bark, \& Cooper, 2006; Krakowski, Czobor, \& Nolan, 2008; Spivak et al., 1997; Swanson, Swartz, \& Elbogen, 2004; Volavka et al., 2004); and reviews (Aleman \& Kahn, 2001; Brieden, Ujeyl, \& Naber, 2002; Glazer \& Dickson, 1998) suggesting superiority of atypical antipsychotics and particularly clozapine in treating violence in patients with psychosis-spectrum disorders. However, previous studies did not perform a meta-analysis of different atypical antipsychotics and therefore could not quantify these difference. All previous reviews and expert opinions pointed to clozapine as being most effective. This quantitative systematic review confirms these statements using all currently available evidence from randomized controlled trials.

Some atypical antipsychotic drugs, especially olanzapine, quetiapine and clozapine are more sedative than haloperidol (Leucht et al., 2013), which could explain their effect on hostility. However, most clinical studies control for the effect of sedation and activation as well as psychosis, which could indicate that the anti-hostility effect was at least partially independent from the sedating, activating and antipsychotic effects of the drug. Moreover, augmentation with benzodiazepine, which does not 
reduce hostility, shows that sedation alone is not responsible for this effect (Correll,Yu, Xiang, Kane, \& Masand, 2017). Indeed, animal work using knock-out mouse models that are insensitive to sedative effects of clozapine, still show efficacy of clozapine in reducing aggression in these animals (Gallitano-Mendel, Wozniak, Pehek, \& Milbrandt, 2008), suggesting the superiority of clozapine and to a lesser degree of other atypicals, may be independent of sedation. Another explanation for the superiority of atypical antipsychotics is their effects on 5-HT receptors. Aggression and hostility have been linked to serotonergic abnormalities both in human and animal studies (Silver, 2001). In patients with psychosis-spectrum disorders, polymorphism of the 5HT2a promoter region is associated with social cognition and anger management (Lo et al., 2010). SSRI's have been suggested to be effective in treating hostility in autism, but for schizophrenia this evidence is lacking (Silver, 2001).

Of course, we need to keep in mind that hostility and violent behaviour in psychosis-spectrum disorders have heterogeneous aetiology, and may include fear, delusional beliefs, command hallucinations, lack of impulse control or comorbid antisocial personality or drug addiction. We should therefore not expect that any given pharmacological treatment will be equally effective in reducing violent behaviour caused by psychosis, impaired impulse control, craving or personality disorder (Volavka \& Citrome, 2008).

\section{Strengths and weaknesses of the study}

This study has several limitations. The search initially included studies that focused on violence or hostility and reported as such in the title or abstract. Studies without any of our keywords in title or abstract may have been missed. However, the cross reference search was performed thoroughly and included other meta analyses and reviews. Second, the PANSS and the BPRS are instruments with different scoring instructions. While both have a similar outcome measure, the scores are not interchangeable. We, however, could not analyse them separately because of the loss in power. Third, the large number of sponsored studies could cause bias. However, the separate analysis of the non-sponsored studies also showed very similar effects. The heterogeneity of the sponsored studies is much higher. Possibly because, in many cases, sponsored studies were conducted to establish a dose-response relationship. Therefore, these studies included several dose arms for the same antipsychotic (e.g., for risperidone and amisulpride), which increased the variation in terms of therapeutic efficacy, and were likely to enhance the variation of therapeutic effects (and increased heterogeneity) with respect to hostility. Studies without sponsorship used established fixed doses of antipsychotic medications, or flexible doses in an established dose range to achieve the best therapeutic effects in individual patients, which could be behind, at least in part, the lower level of heterogeneity in these studies. Finally, a number of studies only 
used one typical antipsychotic as a control versus multiple atypical antipsychotics or dose-groups. Although the $\mathrm{N}$ of the typical antipsychotics is divided by the number of atypical arms, this could cause an over- or underestimation.

Moreover, some limitations of our inclusion criteria should be noted. This review only included studies investigating the medication for one month or more. We did not look at the acute effect of antipsychotic drugs in emergency situations, as these effects may differ from those of maintenance treatment. These studies mostly focus on the first day of treatment and include, for example, the use of short-acting olanzapine IM, short-acting aripiprazole IM, loxapine inhaled, sublingual asenapine and antihistamines. As a recent review towards medication for psychosis-induced aggression or agitation concluded there is only a small amount of evidence and of poor quality (Zaman et al., 2018), future research could compare the effects of medications in emergency situations and long term treatment. Finally, only studies with hostility scores as outcome measure are included and not the studies using physical violent incidents as an outcome measure, as there were too few of those. However, our results seem to be compatible with previous research studying the anti-aggressive effects of typical and atypical antipsychotics in a population of violent patients, showing a superiority for atypical drugs and specifically clozapine (Krakowski et al., 2006; Krakowski et al., 2008; Spivak et al., 1997; Swanson et al., 2004;Volavka et al., 2004).

The strengths of this study, however, are the large number of patients used in the main analysis and the quantification of effect sizes.

\section{Conclusion}

In conclusion, we confirmed previous claims of superiority for atypical antipsychotics as compared to typical antipsychotics in treating/reducing hostility but found only a small effect with high heterogeneity. When we restricted our meta-analysis to only studies that were not industry sponsored, the effect remained largely the same, but heterogeneity disappeared. This suggests that there is indeed a small yet significant advantage. When we restricted our analysis to clozapine versus typical medication, the effect size almost doubled and heterogeneity largely disappeared. Finally, when differentiating between high and low dosed antipsychotics, the high dosed studies had a significantly greater effect on hostility compared with low dose antipsychotics. Although this effect could be partially caused by the clozapine studies in the highdose groups, this possibly indicates that higher dosed atypical antipsychotics are most effective to deal with violent behaviour from patients with psychosis spectrum disorder in daily practice. These results are important for clinicians to help their shared decision making with patients when choosing maintenance treatment, as next to efficacy for psychosis and tolerability, safety for the patient and their environment is an important outcome. 



\section{CHAPTER 7}

Summary 



\section{Summary}

Aggression is a major problem in mental health care and affects patients, their surroundings, health care workers and the society in general. Despite years of research on this topic, there are still many clinical decisions not based on evidence. The aim of this thesis is to contribute to the current knowledge about aggression in psychiatry with clinically relevant results and insights. The focus is on three main themes: prevention, epidemiology and treatment. These themes are addressed in the five studies that are presented in this thesis (Chapters 2-6). In this chapter, we summarize the findings of the previous five chapters.

\section{Chapter 2. Warning signs prior to aggressive behaviour in child psychiatric units}

The motivation for the second chapter was that there are no known warning signs prior to aggressive incidents in children. Knowledge about warning signs enables professionals to observe these kinds of behaviours and anticipate on this by, for example, using preventive interventions. While in adult mental health care, there is some knowledge on changes in patients' behaviour prior to aggression, there is limited evidence within child psychiatric facilities.

The aim of this study is therefore to determine the most prevalent early warning signs prior to aggressive incidents in a sample of children from 4 to 12 years of age in clinical and semi-clinical psychiatric facilities. To identify these warning signs, we used the Proactive monitoring of Aggression in Children Tool (ProACT (Valenkamp, 2011)), which was developed for staff to report aggressive incidents. For this analysis, the open-ended question was used: "which warning signs did you observe?" We also incorporated an indication of the severity of the incident. Data were collected from 26 inpatients units within 4 child psychiatric settings and 2 child welfare services. 236 children were admitted within these units of which 131 (56.4\%) were involved in one or more aggressive incidents as reported with the ProACT.

A total of 1068 incidents were reported of which 575 (53.8\%), one or more warning sign was entered. 62 (10.8\%) incidents were categorized as "severe," meaning there were physical consequences for staff. Because this was an open question, a coding process was needed to identify the most prevalent warning sings out of all these descriptions. The coding process consisted of six steps: 1) open coding, 2) expert review round I, 3) intercoder agreement test I, 4) expert review round II, 5) intercoder agreement test II, 6) final coding. This process resulted in a total of 16 warning signs, divided into 4 categories. The most common warning sign was "restlessness" which 
was mentioned in 230 (21.2\%) of the incident reports. Second was "not listening" with 165 (15.2\%) entries. Third was “anger" with 106 (9.8\%) entries. These three signs were also most prevalent prior to the incidents marked as "severe."

Although this is a first step in identifying warning sings in children, and further research towards the clinical utility, interrater reliability and the predictive capacity of this list is needed, this list could be helpful in daily practice for nurses to become aware of changes in children's behaviour that can predict aggression. Also, the signs can be integrated in aggression-evaluation forms, personal de-escalation plans and could become part of the training for nursing students and professionals to observe and recognize these possible signs of escalation and subsequently act on it.

\section{Chapter 3. Kennedy Axis $\vee$ : clinimetric properties assessed by mental health nurses}

The third chapter is focussed on risk assessment instruments. These instruments are important for risk management in clinical psychiatry as they quantify the risk for aggressive behaviour. For a seclusion reduction programme in a large university hospital, we set out to select clinically useful instruments. A systematic review of the literature found 19 available instruments of which, after appraising the methodological quality, three could be useful for short-term risk assessment. However, another instrument, the Kennedy Axis V, was identified. This instrument was advised as best practice in the Netherlands (Inspectorate of Health, 2011). As this instrument uses both dynamic and static risk factors, assesses multiple risk factors and can be used for outcome measurement, the Kennedy Axis V has many benefits compared with contemporary risk assessment instruments. However, only a few studies systematically evaluated its clinimetric properties.

The aim of this study is to evaluate the interrater reliability and clinical utility of the Kennedy Axis V when used by nurses in daily care of patients with mental illness. This cross-sectional study was conducted in an adult inpatient and outpatient psychiatric unit and in one adolescent inpatient unit at a university hospital in the Netherlands. Interrater reliability was measured based on the independent scores of two different nurses for the same patients. The clinical utility was evaluated by means of a questionnaire.

The overall results revealed a substantial level of agreement between nurses as both the intraclass correlation coefficient (ICC) and Pearson's $r$ were 0.79. It took nurses on average 15 minutes to complete the instrument but this decreased after nurses had scored the instrument more often. Some challenges were identified, including difficulties with scoring the instrument and using interventions based on the scores. 
On the adolescent ward, the nurses reported problems with using the instrument soon after the implementation. After one month, it was decided to stop using the instrument. To gain a deeper understanding of these problems, additional data were collected by means of two focus group interviews. Six nurses (50\%) from the adolescent ward participated.The major problem was the distinction between psychiatric problems and developmental and childhood issues. The manual did not provide enough information on how to use the scale in an age-specific way. It was, however, useful to have some anchor points to observe aggressive behaviour and the instrument was efficient to use.

The Kennedy Axis V can be a useful instrument for short-term risk assessment but the implementation should be accompanied by specific strategies. The implementation of risk assessment instruments is time-consuming and needs careful planning of multifaceted and tailored implementation strategies (Grol \& Grimshaw, 2003; Otto \& Douglas, 2011). Using these strategies, the Kennedy Axis V can contribute to risk assessment and possibly in the prevention of aggression, suicide, self-harm, severe self-neglect and enhanced objectivity in clinical decision-making.

\section{Chapter 4. Aggressive behaviour, hostility and associated care needs in patients with psychotic disorders: a 6-year follow-up study}

Aggression and hostility are associated with each other and with other factors, such as suicidality, impulsivity and childhood trauma. However, these variables are often studied in retrospect. Therefore, in the fourth chapter, we report the results of the Positive And Negative Syndrome Scale (PANSS (Kay et al., 1987)) hostility item, self-reported maltreatment to others, and the Camberwell Assessment of Need Short Appraisal Schedule (CANSAS (Phelan et al., 1995)) safety needs ("safety-to-others") analysis of the Genetic Risk and Outcome of Psychosis (GROUP) study (Korver et al., 2012), a naturalistic, multicentre, six-year prospective cohort study of patients with non-affective psychotic disorders.

The aim is to analyse the incidence, persistence, risk factors and attributable fraction for hostility, self-reported aggressive behaviour and associated care needs in a population of patients with psychosis. Regression analysis was used to analyse the association between these outcome measures and the hypothesized risk factors. The population attributable risk fraction (PAF) was used to calculate the proportion of the outcome that could potentially be prevented if previous expressions of adverse behaviour were eliminated.

A total of 1119 patients were included at baseline of whom 188 (20\%) had a history of maltreatment to others. The yearly incidence of hostility was $2.8 \%$, the incidence of a safety-to-others need was $0.8 \%$ and for maltreatment this was $1.8 \%$. 
Maltreatment was significantly associated with safety-to-others (HR $6.28 p=.00)$ and vice versa (HR $4.50 p=.00)$, corrected for safety-to-self. Safety-to-others (HR 3.58 $p=.00)$ and maltreatment $(1.88 p=.04)$ were significantly associated with hostility. In the Cox regression model of maltreatment, there were no significant associations with the PANSS items hostility, poor impulse control, excitement, tension and lack of cooperation. In the Cox regression model for safety-to-others, there was an association with the PANSS items poor impulse control (HR $2.38 p=.00$ ), hostility (HR $1.56 p=.03)$ and lack of cooperation (HR .53 $p=.02)$ but not with excitement (HR $1.11 p=.64)$ or tension (HR $1.11 p=.56)$. In the Cox regression model of hostility, there were significant associations with both poor impulse control (HR $1.59 p=.00)$, excitement (HR $1.31 p=.02)$ and lack of cooperation (HR $1.63 p=.00)$ but not with tension (HR $1.16 p=.13)$. Safety-to-others was associated with previous hostility and vice versa, but only $18 \%$ of incident safety-to-others were attributable to previous hostility while $26 \%$ was attributable to impulsivity. Hostility and maltreatment, but not safety-to-others, were associated with childhood adversity. Hostility, maltreatment and safety need were all associated with number of unmet needs, suicidal ideation and male sex. Neither safety-to-others, maltreatment nor hostility was associated with premorbid adjustment problems.

This survival analysis of hostility, self-reported aggressive behaviour and associated care needs, affirmed many of the known risk factors. There were however, differences between the outcome measures. Aggression in patients with psychosis appears to be a complex concept with different origins, as these, and other studies have shown. We found childhood adversity but not premorbid problems to be associated with aggressive behaviour. Clinical symptoms can index aggressive behaviours years later, providing opportunities for preventive strategies and a possible decrease of incidents of aggression.

\section{Chapter 5. Hostility and aggressive behaviour in first episode psychosis: results from the OPTiMiSE trial}

Studies show that First Episode Psychosis (FEP) patients are specifically at risk for aggressive behaviours (Large \& Nielssen, 2011; Winsper et al., 2013). However, many questions considering associated clinical factors and the effect of medication on aggressive behaviour remain unanswered. This is why the fifth chapter is aimed to determine clinical factors related to hostility and disturbing and aggressive behaviours and to examine the effect of amisulpride and olanzapine on these behaviours in FEP.

Results from phase I and II of the Optimization of Treatment and Management of Schizophrenia in Europe (OPTiMiSE) trial are used (Kahn et al., 2018; Leucht 
et al., 2015), which included 512 FEP patients in 27 sites across Europe and Israel. Patients start with 4 week open label amisulpride in flexible dosage (phase I) and continue to a 6- week double blind phase, if they are not in remission, where there are randomized to either amisulpride or olanzapine, also in flexible dose (phase II).

For the current analysis, the main outcome measures are the PANSS hostility item and Personal and Social Performance scale (PSP (Morosini et al., 2000)) disturbing and aggressive behaviours domain. The intention-to-treat sample at baseline $(\mathrm{N}=446)$ was used to determine the clinical factors associated with hostility. PANSS items that were previously associated with hostility (excitement, poor impulse control, tension and uncooperativeness) were included, as well as some items to see if positive symptoms are associated with aggression in this sample (delusions, hallucinatory behaviour and unusual thought content). For the medication analysis, patients with baseline hostility $>1$ are included in the hostility subsample, in line with previous work. PANSS hostility and PSP disturbing and aggressive behaviours mean values and mean differences were calculated.

Both hostility and disturbing and aggressive behaviour were significantly associated with each other as well as with PANSS items delusions, hallucinatory behaviour, excitement, tension, uncooperativeness, unusual thought content, lack of judgement and insight and poor impulse control. Remarkably, while positive symptoms are related to hostile and aggressive behaviour, the association is higher for impulsiveness, excitement and uncooperativeness. 185 (41.5\%) patients had baseline hostility $>1$. During phase I, the hostility scores significantly improved and this effect remained significant after correcting for baseline positive symptoms. We found no significant difference between olanzapine and amisulpride in the second phase of the trial.

The prevalence of hostile and aggressive behaviour in this selected sample was low. Behavioural symptoms, such as impulsivity, excitement and uncooperativeness are more relevant for hostile and aggressive behaviour than positive symptoms. In the hostility subsample, amisulpride was effective in the treatment of aggressive behaviours.

\section{Chapter 6. Efficacy of typical and atypical antipsychotic medication on hostility in patients with psychosis-spectrum disorders: a review and meta- analysis}

Antipsychotic medication can be an important intervention in the treatment of aggressive behaviour in patients with psychotic disorders as it has been suggested that these prevent or reduce aggressive behaviour. Specifically clozapine should be effective 
for aggressive behaviour in patients with treatment-resistant psychotic disorders. There is, however, no consistent evidence to make a rational decision as to which type of antipsychotic is most effective in reducing aggressive behaviour and even the superiority of clozapine is not well documented. Since very few studies, unfortunately, have investigated the influence of different antipsychotic drugs on actual rates of aggression incidence, the hostility item of the PANSS is often used as a proxy to give an indication of the aggressiveness of the patient. Therefore, the sixth chapter describes a systematic review and meta-analysis with the aim to provide support for a rational decision on which type of antipsychotic medication most effectively reduces hostility.

The initial literature search resulted in 1395 studies. After screening, 18 suitable randomized studies comparing typical to atypical antipsychotics for at least 4 weeks in patients with psychotic disorders were included. Data from 6799 patients were used. The used atypical antipsychotics included amisulpride, clozapine, olanzapine, quetiapine, risperidone and ziprasidone. These were compared with the typical antipsychotics haloperidol, chlorpromazine and perphenazine. Results show a small (Hedges' $g=.26$ ) but significant effect for atypical as compared to typical antipsychotics. Heterogeneity was high $\left(I^{2}=92.84\right) .14$ studies $(83 \%)$ were sponsored by pharmaceutical companies but the effect size remained similar when separately analysing sponsored (Hedges' $g=.262, p=.047$ ) and non-sponsored studies (Hedges' $g=.248, p=.340)$. When differentiating between high and low-dose studies, the highdose group showed a significant difference between typical and atypical antipsychotics (Hedges' $g=.567, p=.001$ ) whereas the low-dose group did not (Hedges' $g=.023$, $p=.871)$.An analysis comparing clozapine to typical antipsychotics showed a moderate effect size (Hedges' $g=.415, p=.0000)$ with low heterogeneity $\left(I^{2}=19.16\right)$.

These results support previous claims of superiority of atypical antipsychotics as compared with typical antipsychotics in treating or reducing hostility but we found a small effect size and high heterogeneity. When we restricted our analysis to clozapine versus typical medication, the effect size almost doubled and heterogeneity largely disappeared. These results are important for clinicians to help their shared decision making with patients when choosing maintenance treatment, as next to efficacy and tolerability, safety for the patient and their environment is an important outcome. 



\section{CHAPTER 8}

\section{General discussion}





\section{General discussion}

The aim of this thesis is to contribute to the current knowledge about aggression in psychiatry with clinically relevant results and insights. There are three main themes: prevention, epidemiology and treatment. The preventive section is focussed on warning signs prior to aggressive behaviour and on the clinimetric properties of a structured observation instrument (Chapters 2\&3). In the epidemiology section, we set out to identify incidence rates, prevalence, and risk factors for aggression in patients with psychotic disorders (Chapters $4 \& 5$ ). The main focus of the treatment section is the efficacy of antipsychotics on aggressive behaviour (Chapters $5 \& 6$ ).

In this final chapter, we discuss the most important findings of this thesis according to the three main themes. Both the scientific contribution and suggestions for clinical application of the findings are discussed. Table 1 summarizes the most important implications for clinical practice. Later on in this chapter, we discuss the general limitations of the findings, followed by some relevant recent developments, suggestions for future research and the general conclusion.

\section{Prevention}

\section{Warning signs prior to aggression in children}

In the second chapter, we concluded that common warning signs can be observed prior to aggressive behaviour in children. While some research has identified warning signs in adult psychiatry (Fluttert, Van Meijel, Bjorkly, Van Leeuwen, \& Grypdonck, 2013; Steinert, 2006), the identification of warning signs prior to aggressive incidents in child psychiatry is new. The identification of the 16 most common warning signs in child psychiatry is a first but important step towards a more evidence-based approach in the prevention of aggression in child psychiatry. Future research is needed to test the clinical applications and predictive capacity of the warning signs. Routinely collected warning signs, for example in incident report forms, can help in unravelling causes and patterns of aggressive behaviour. The identification of these warning signs can also be a first step in the development of a risk assessment instrument, such as the Bröset Violence Checklist (BVC (Almvik \& Woods, 1999)), specifically for child psychiatry and this could be a target for future research.

In clinical practice, the identified warning signs can be applied on three different levels. First, the signs could be incorporated in children's personal deescalation plans. The child, nurse, and for example the parents, could collaborate on 
selecting the signs that are applicable for that child. Signalling warning signs and discriminating them from antecedents or provocations can be difficult and therefore, an overview of the most prevalent warning signs can help in selecting personal warning signs. Whilst only the most prevalent signs were listed, specific signs could be added. A personal de-escalation plan that includes warning signs can help patients and their surroundings in the early recognition of aggression. Second, the signs could be added to incident report forms. For example with a multiple choice option after the question: "which warning signs did you observe prior to the incident?" Analysis of these report forms can be useful at patient-level, for children with persistent aggressive behaviour to detect patterns. On ward-level, analysis of aggressive incidents, including the warning signs, can be used for policy-making. Third, the list of warning signs could be used during training sessions to teach health care workers to recognize changes in children's behaviour that precede aggressive incidents. This could especially be useful for inexperienced colleagues that are developing their skills in detecting emerging aggression.

\section{Risk assessment instruments}

In the third chapter, we reported that a structured observational tool, like the Kennedy AxisV (Kennedy, 2003b), can be reliable and clinically useful in practice when carefully implemented. While it was already known that this instrument could be used for risk assessment (van de Sande et al., 2017), these results contribute to the existing literature on the Kennedy Axis $\mathrm{V}$ that the interrater reliability and clinical utility are sufficient. We found that careful implementation of this instrument is crucial and we concluded that it was challenging to use preventive interventions that are linked to the outcome of the Kennedy Axis V. This confirms previous work on risk assessment instruments, where both the difficulties with implementation (Hoge, 2010; Webster \& Hucker, 2007) and application of preventive interventions (Kaunomaki et al., 2017) have been reported. Another novel finding was that there were problems with scoring the instrument on the adolescent ward while, according to the author, the instrument should be useful across all age groups (Kennedy, 2003b). As far as we are aware, this was the first evaluation of the Kennedy Axis V within an adolescent unit.

The use of the Kennedy Axis $\mathrm{V}$ is recommended in adult psychiatry, but with a specific implementation strategy. In contrast with most other risk assessment instruments, the Kennedy Axis V can also be used as a routine outcome instrument and incorporates other adverse outcomes next to aggression. Therefore, the instrument can be used for treatment planning and enhance objectivity in clinical decisionmaking. The difficulties with scoring the instrument can probably be resolved by careful implementation and prolonged experience with the use of this instrument. Some implementation strategies are advised in Chapter 3, such as training sessions, 
newsletters, pilot testing and careful planning of the implementation process. An important strategy is the incorporation of the instrument into clinical processes. Risk assessment scores should always be interpreted together with professional judgement, ideally in a multidisciplinary evaluation. This should be incorporated into treatment planning and subsequently lead to preventive interventions, if there is an enlarged risk for adverse outcomes such as aggression.

\section{Epidemiology}

\section{Incidence and prevalence}

The fourth chapter reports incidence data of aggressive behaviours in patients with psychotic disorders and the fifth chapter reports prevalence data of aggression in First Episode Psychosis (FEP). Both chapters contribute to numerous of previous findings. However, as discussed in Chapter 1, aggression rates are difficult to compare. Moreover, data from both Chapters 4 and 5 consist of selected samples, limiting the generalizability of these findings. However, whilst more prospective studies, reporting incidence data, are needed, the current findings contribute to the body of knowledge that is needed to understand the phenomenon of aggression.

The fourth chapter is a valuable contribution as most earlier studies within this population are retrospective and report prevalence rates. Incidence is difficult to compare with prevalence but we can compare meta-analytic prevalence data with the baseline rates. The prevalence of Positive and Negative Syndrome Scores (PANSS (Kay et al., 1987)) hostility scores $>1$ was $18 \%$ during the past week, $12.8 \%$ of patients had a Camberwell Assessment of Need Short Appraisal Schedule (CANSAS (Phelan et al., 1995)) safety-to-others met or unmet need during the past month and $20 \%$ had a history of lifetime maltreatment to others. However, hostility scores of 1 indicate minimal presence of hostility (Kay et al., 1987) and is therefore not comparable with incidents of aggression. Lifetime maltreatment is evidently higher compared with point prevalence rates. Therefore, in comparison with earlier work that report point prevalence rates for different types of aggression around $18.5 \%$ in patients with psychotic disorders (Witt et al., 2013), we believe the current rates are somewhat lower.

The fifth chapter reports prevalence of PANSS hostility within a sample of patients with First Episode Psychosis (FEP). We found hostility scores $>1$ in $41.5 \%$ of patients at the baseline study visit which is lower compared with an earlier Randomized Clinical Trial (RCT) with FEP patients, where the prevalence of baseline hostility scores $>1$ was $60.1 \%$ (Volavka et al., 2011). Hostility scores $>3$, indicating "overtly 
hostile attitudes, verbal abuse and aggression" (Kay et al., 1987), were present in 9.4\% of patients at baseline. This is lower compared with meta-analytic data that indicate prevalence rates of aggression in FEP $34.5 \%$ prior to contact with health services or during the initial treatment period (Large \& Nielssen, 2011).

\section{Risk factors}

Chapters 4 and 5 report risk factors associated with different aggression outcomes, contributing to many previous studies that try to unravel the factors that place people at risk for developing aggressive behaviour. In this section, we will discuss two categories of risk factors: clinical symptoms and early adversity.

First, clinical symptoms associated with aggression in patients with psychotic disorders, discussed in Chapter 4, were impulsivity and excitement, consistent with earlier findings and found uncooperativeness associated with the outcome measures, in contrast to earlier findings (Witt et al., 2013). However, not all aggression outcome measures were associated with these symptoms. This appears to be an indication of the complexity of aggression and the subtle differences between the outcome measures. In Chapter 5 we confirmed that impulsivity and lack of judgement and insight are associated with aggressive or hostile behaviour in FEP (Moulin, Golay, et al., 2018; Volavka et al., 2016). Additionally, we found excitement and uncooperativeness associated with hostility and aggression. Chapter 5 also included other positive symptoms and found that hallucinations, delusions, and unusual thought content were associated with hostility and with aggressive behaviour, whilst earlier findings are contradictory (Coid et al., 2013; Langeveld et al., 2014; Large \& Nielssen, 2011; Witt et al., 2013).

In clinical practice, these risk factors are important as they can predict aggressive behaviour even years later, suggesting they may represent trait-like risks. Health care workers should have some knowledge on risk factors for aggressive behaviour as this enables early recognition. Specifically in patients with additional risk factors, such as substance abuse (Fazel et al., 2009), these risk factors should be considered in clinical decision making. Assuming causality, treatment of these symptoms could reduce the risk and this should be discussed with patients in shared decision making. One risk factor of specific concern for clinical practice is impulsivity. Impulsivity was associated with aggression but also with suicidality, although again with some differences between the outcome measures (Chapter 4). This is in line with earlier work, suggesting that impulsivity is the mediator between aggressive and suicidal behaviour (Witt et al., 2014). Therefore, impulsivity is an important risk factor for clinical practice that should be considered in a routine matter.

Second, in Chapter 4 we found childhood trauma associated with aggressive behaviour while premorbid problems were not associated. This confirms previous 
evidence (Bosqui et al., 2014; Swanson et al., 2006; Witt et al., 2013) and contributes to the understanding of the complexity of aggression aetiology and potential treatability, to the degree that the sequelae of trauma are treatable and with that possibly the way it impacts aggression risk. More research is needed to find out if there is causality and if treatment of the sequelae of trauma indeed reduces aggression risk and can give insight in the aetiology of aggression. This also emphasizes the importance of the prevention of childhood trauma in the general population. People with a history of childhood trauma are at risk for developing both a psychotic disorder and aggressive behaviour (Gibson, Alloy, \& Ellman, 2016; Isvoranu et al., 2017; Oakley et al., 2016).

In clinical practice, childhood trauma is a more complicated risk factor than symptoms such as impulsivity as evidently, previous events can never be made undone. However, the psychological sequelae of trauma are treatable with psychotherapy and the risk of aggression associated with trauma may also be treatable if it is affected by the same mechanisms targeted by psychotherapy. Whilst causality has to be determined, the treatment of the sequelae of trauma in patients is important either way. In patients with psychotic disorders, childhood trauma is often not recognized and Posttraumatic Stress Disorder (PTSD) is under-diagnosed (Grubaugh, Zinzow, Paul, Egede, \& Frueh, 2011; Lommen \& Restifo, 2009). In line with previous suggestions (Horowitz, Guyer, \& Sanders, 2015), this urges a need to screen patients for trauma in a routine matter, specifically those with persistent aggression, and consider the treatment of the psychological sequelae of trauma.

\section{Treatment}

\section{Amisulpride and olanzapine}

In Chapter 5, we analysed data from the Optimization of Treatment and Management of Schizophrenia in Europe (OPTiMiSE) trial, and found that amisulpride was an effective antipsychotic in reducing hostility in FEP patients during the first four weeks of treatment. This effect is, at least partially, independent from other baseline positive symptoms. Earlier work also indicated a decrease in hostility scores in patients using amisulpride but found olanzapine superior (Volavka et al., 2011), as olanzapine was also superior to other antipsychotics in a later study, although amisulpride was not used in that study (Volavka et al., 2014). Interestingly, the dataset used by Volavka et al. in 2014 was also used in an earlier analysis that incorporated aggression as an outcome measure (the MacArthur Community Violence Interview) which did not find any of the atypical agents superior (Swanson et al., 2008; Volavka et al., 2014). There are several plausible explanations for this apparent discrepancy and this emphasizes again 
that there are differences between hostility and aggression (Volavka et al., 2014). Olanzapine was used in the randomized second phase of the OPTiMiSE trial, but we could not find a significant difference between amisulpride and olanzapine due to the low sample size and a ceiling effect since the mean scores at baseline phase II were low and there could only be little improvement. Whilst this could be a target for future research, the finding that amisulpride was effective in reducing hostility in the first phase of the trial is an important contribution to the current evidence about treatment of patients with FEP and aggressive behaviour.

Clinicians should consider amisulpride for patients with FEP to reduce hostility and aggressive behaviour during the first weeks of treatment. Amisulpride is not licensed in the USA and some European countries (Kahn et al., 2018), despite evidence for its efficacy against psychosis (Huhn et al., 2019). Therefore, this recommendation is limited to the countries where amisulpride is available. While clozapine is most effective against hostility (Chapter 6), current clinical guidelines recommend clozapine for patients that were non responsive to two adequate trials of different antipsychotics (Warnez \& Alessi-Severini, 2014) and is therefore no option for FEP patients. In Chapter 5, we could not determine a difference between olanzapine and amisulpride. While olanzapine may be superior to amisulpride in treating hostility (Volavka et al., 2011), olanzapine induces a higher risk for weight gain compared with amisulpride (Huhn et al., 2019). We therefore believe that amisulpride should be considered for the treatment of FEP patients with aggressive behaviour, specifically when olanzapine is contraindicated because of the risk of weight gain.

\section{Clozapine}

In Chapter 6, the superiority of atypical antipsychotics over typical antipsychotics in reducing hostility was confirmed through a meta-analysis. Specifically clozapine was superior, in line with previous work (Frogley et al., 2012; Glazer \& Dickson, 1998; Spivak et al., 1997; Volavka \& Citrome, 2018). This finding is not new but adds a layer of confidence to the evidence through a meta-analysis. A novel and interesting finding was the significant difference in the high-dosed group in favour of atypical antipsychotics while there was a non-significant difference in the low-dosed group. Since there is limited evidence on the effect of antipsychotic dose on hostility and aggressive behaviour, but increasing the antipsychotic dose for patients with aggressive behaviour is a frequently used intervention in clinical practice, this urges a need for future research.

This confirmation of the effectiveness of clozapine for the treatment of hostility in patients with psychotic disorders implicates that clinicians should consider clozapine for the treatment of patients with psychotic disorders and persistent hostile or aggressive behaviour. Clozapine is still under-prescribed in clinical practice because 
of concerns about serious adverse effects such as agranulocytosis, hyperglycaemia and the neuromalignant syndrome (Warnez \& Alessi-Severini, 2014). The risk for these side effects should be considered in light of the effectiveness of clozapine as there are also many negative consequences linked to hostile and aggressive behaviour. Specifically the poorer social functioning that is linked to hostile behaviours (Lahera et al., 2015) could have a major impact on the daily lives of patients. Therefore, clozapine should be considered for patients with psychotic disorders and persistent hostile or aggressive behaviour, in shared decision making processes.

As for the pharmacological implications of Chapters 5 and 6, two additional remarks should be made. First, clinicians should consider treatment adherence next to the effectivity of antipsychotics against hostility. Treatment discontinuation has been linked to hostility (Volavka et al., 2016), urging the need for clinicians to choose an antipsychotic in shared decision making with the patient and to make an effort in ensuring treatment adherence (Nieuwlaat et al., 2014). Treatment adherence could also be a motivation to choose a Long Acting Injectable (LAI) instead of clozapine or amisulpride, which are both unavailable as LAI while olanzapine is. Second, the effectivity of antipsychotics against hostile or aggressive behaviour in patients with psychotic disorders may depend on the aetiology of aggression that can include fear, delusions, impulsivity, substance abuse or personality disorders. We cannot assume that pharmacological treatment is equally effective in reducing violent behaviour with different aetiology (Volavka \& Citrome, 2008). In patients with persistent aggression, clinicians should make an effort to unravel the aetiology of aggression, using clinical reports or risk assessment instruments when available, and discuss these behaviours and treatment options with patients, carers or relatives.

Table 1. Implications for clinical practice derived from the findings in this thesis

\begin{tabular}{|c|c|c|}
\hline Theme & Chapter & Implications for clinical practice \\
\hline \multirow[t]{2}{*}{ Prevention } & 2 & $\begin{array}{l}\text { The list with the most prevalent warning signs prior to aggressive incidents } \\
\text { should be applied in child psychiatry. }\end{array}$ \\
\hline & 3 & $\begin{array}{l}\text { Instruments for structured observation can assist in clinical risk assessment } \\
\text { but should be carefully implemented using specific strategies. }\end{array}$ \\
\hline \multirow[t]{2}{*}{ Epidemiology } & 4 & $\begin{array}{l}\text { Clinicians should consider behavioural symptoms, such as impulsivity, } \\
\text { excitement, lack of cooperation, and hostility as these can predict } \\
\text { aggressive behaviour even years later, suggesting they may represent trait- } \\
\text { like risks. }\end{array}$ \\
\hline & 4 & $\begin{array}{l}\text { Childhood trauma is an important risk factor for aggressive behaviour in } \\
\text { patients with psychosis and treatment of the psychological sequelae of } \\
\text { trauma should be considered. }\end{array}$ \\
\hline \multirow[t]{2}{*}{ Treatment } & 5 & $\begin{array}{l}\text { Amisulpride could be used for patients with FEP to reduce hostility and } \\
\text { aggressive behaviour during the first weeks of treatment. }\end{array}$ \\
\hline & 6 & $\begin{array}{l}\text { Clozapine is the most effective antipsychotic for the treatment of patients } \\
\text { with psychosis and persistent hostile or aggressive behaviour. }\end{array}$ \\
\hline
\end{tabular}




\section{Limitations}

This thesis has two general limitations. First, aggression is a complex concept. However, the approach throughout this thesis is rather dichotomous: aggression is either present or absent. Besides the use of the, mostly dichotomized, PANSS hostility item and the Kennedy Axis $\mathrm{V}$ that incorporates a severity scale, there is no distinction in aggression based on aetiology or a difference in severity. Although dichotomization was needed for the procedures and we had no specific aggression outcomes available in some chapters, analysing only the presence or absence of aggression does not reflect the actual complexity of this phenomenon. Moreover, while treatment is one of the main themes in this thesis, the focus is only at the pharmacological treatment while there are many more treatment options involved. However, this does point out directions for future research.

Second, whilst the scope of this thesis is multidisciplinary, the focus is on clinicians and nurses. User experiences were not incorporated in the design of this thesis. Discussion of this topic, and some results of this thesis, with health care workers and patients, with and without experiences of aggression, could have been interesting, both at the directions for research topics and regarding the implementation of research results into practice. Incorporating user experiences on this matter could contribute to a more diverse approach of the problem. For instance, a problem that should be discussed with patients is the possible contribution of studying aggression in psychiatry to stigmatization, as discussed in Chapter 1.

\section{Recent developments}

During the recent years, there have been some important new developments regarding both research and clinical practice. Although this was beyond the scope of this thesis, these developments shed light on what could be important for future directions in research and in practice.

First, an important development in Dutch psychiatry is the focus on seclusion reduction. Traditionally, seclusion rates are higher in the Netherlands compared with other countries, while restriction and coercive medication rates are lower (Janssen et al., 2008; Noorthoorn et al., 2015). Traditional seclusion rooms offered a very bleak and threatening environment to patients and although much debated, there is no sufficient evidence for the effectiveness of seclusion (Sailas \& Fenton, 2000). This is why mental health care facilities have worked on a reduction of the use of seclusion and other coercive interventions. In the Netherlands, from 2006-2012, there was a government funding for seclusion reduction programmes. Within these programmes, a number of interventions and best practices for seclusion reduction were developed 
and implemented. This has led to a reduction of seclusion in the years following these funded programmes (Noorthoorn et al., 2016), although it is difficult to compare seclusion rates as there was no structured and uniform collection of data before the start of this project. There is some recent evidence that seclusion rates are increasing years after the projects (Mann-Poll et al., 2018), but conclusions can not yet be drawn as this could also be a minor trend.

A recent development following these initiatives are the High- and Intensive Care (HIC) units in Dutch psychiatry. HIC units include an Intensive Care Unit (ICU) with a High Security Room that can be locked but is only used as a last resort and always with a nurse within eyesight, following the principle that severely ill patients should never be left alone. HIC units have a recovery-oriented atmosphere and incorporate the use of a stepped-care model (van Mierlo, Bovenberg, Voskes, \& Mulder, 2013). A HIC-monitor has been developed to evaluate HIC units across the Netherlands and a first evaluation of this instrument found good clinimetric properties (van Melle et al., 2019). This HIC-monitor enables comparison across wards and can assist in the implementation of the HIC model and for research purposes.

Second are some technological innovations. For example,Virtual Reality (VR) can be used for both treatment of aggressive behaviour and training for professionals. Patients can be trained in VR real-life situations where they can practice de-escalating techniques and learn to recognize and control aggressive impulses (Klein Tuente, Bogaerts, van, \& Veling, 2018). Preliminary results of studies usingVR as a therapeutic element show a reduction in anger and decrease in aggression (Dellazizzo, Potvin, Bahig, \& Dumais, 2019). Another useful application of VR is in clinical training for health care professionals. Specifically for professionals that have experienced less aggression in the past year, VR was could be an effective training method (Buiter et al., 2019) and a useful contribution to traditional training sessions. Next to VR, the use of machine learning techniques for clinical risk assessments appears promising. Recently, a multi-variable prediction model, based on clinical notes in patients' electronic files, has been developed and validated (Menger, Spruit, van Est, Nap, \& Scheepers, 2019). This risk assessment can be automatically derived from the daily clinical notes and could be an addition to current risk assessment instruments and the clinical expertise of the professional. Another technological innovation is the use of biosensors. Biosensors, such as wearables, can help in the early detection of aggressive behaviour by registering heart rate and skin conductance (de Looff et al., 2019).

Third, some pharmacological innovations deserve mention. High dose omega-3 fatty acids could have effect on aggressive behaviour (Choy \& Raine, 2018; Gajos \& Beaver, 2016). For the short-term treatment of aggression in emergency situations, loxapine can now be administered through inhalation. There is evidence that inhaled loxapine is efficient for the treatment of agitation and can be used when 
fast administration is needed (McDowell et al., 2019; Pacciardi, Calcedo, \& Messer, 2019). Inhalation could be less traumatic and painful for patients and a safer alternative for needles. However, patients should be willing to inhale the loxapine and therefore its use may specifically be as self-medication. This is also an excellent research topic to incorporate user experiences in as to evaluate the situations in which loxapine should be considered.

\section{Future research}

Following the results described in this thesis, there are three main directions for future research. The first suggestion addresses the problem with the implementation of risk assessment in clinical practice and the use of interventions accordingly. For an instrument to be of use, the assessment combined with the professional judgement should lead to clinical decisions, including preventive interventions, when there is an enlarged risk. However, the use of interventions derived from risk assessment scores is challenging, as described in Chapter 3, and in earlier work (Kaunomaki et al., 2017). There is limited evidence for some interventions that are considered a best practice, such as de-escalation techniques (Du et al., 2017; Gaynes et al., 2017; Hallett \& Dickens, 2017). The lack of evidence for preventive and de-escalating interventions could be because of the inherent methodological challenges. Action research could be an appropriate method to investigate how risk assessment can be incorporated more efficiently into clinical processes, how assessments could lead to tailored interventions, which preventive interventions and de-escalation techniques are used in practice and if these are effective.

Whilst there is a lot of emphasis on de-escalation, there is more to the prevention of aggression than just interventions. Aggression is relational and contextual and also depends on the degree to which the clinical milieu of the particular psychiatric setting allows for a safe, non-paternalistic and empowering attitude that will help in accommodating patients. A shift from a professional-driven process to a patient-driven dialogue has been suggested (Terkelsen \& Larsen, 2016). Empathic interactions and a relational approach contribute to a recovery-oriented atmosphere with meaningful interactions. This involves more than following a protocol and is not just an intervention or a de-escalation technique as these are not used to achieve a specific outcome, such as the prevention of aggression. In forensic psychiatry, findings suggest lower recidivism in patients that received relational care in combination with voluntary aftercare after discharge (Schaftenaar, van Outheusden, Stams, \& Baart, 2018) but studies in general and short-term inpatient psychiatry are needed. Specifically of interest are situations where both patients and health care workers are frustrated or afraid and empathic interactions are challenging. 
Second, we observe important changes in mental health service environments that we predict will have a beneficial effect on the incidence of aggressive behaviour. For example, the replacement of the traditional seclusion rooms to IC units in the Netherlands with a focus on relationships in both design and professional attitude and with a recovery-oriented treatment environment. We predict that observational research will show a sharp decrease in aggressive incidents with the advent of these novel de-escalating environments in mental health settings in the Netherlands. Whilst there is some evidence for the HIC monitor, used for implementation and evaluation across HIC units (van Melle et al., 2019), future research is needed for the HIC model to be developed further. This should include user experiences from patients and relatives, and a specific focus on the experiences for nurses, as one-to-one caring for severely ill patients in an IC unit indicates a major shift from traditional psychiatric nursing on acute wards.

The third suggestion for future research is treatment. In Chapter 5 and 6, we concluded that more research is needed on the pharmacological treatment of aggression. We propose an RCT, focused on the effects of antipsychotics on hostility and aggression in patients with psychotic disorders. In Chapter 4, the data from the OPTiMiSE trial was unfortunately not suited to determine the dose-effect of antipsychotics or the effect of concomitant benzodiazepine use on hostility and a difference between olanzapine and amisulpride in the second phase of the trial could not be established.A double-blind trial including randomization to olanzapine, amisulpride and clozapine among patients with psychotic disorders could be proposed. Set dosages of these antipsychotics should allow for testing the hypothesis that higher dosages of antipsychotics have more effect on antipsychotics. Regulated benzodiazepine use enables testing whether benzodiazepines reduce aggressive behaviour due to sedation or whether the decreased inhibition causes aggressive behaviour. This trial should include both patients with FEP and more persistent psychotic disorders to compare any differences. Ideally, this trial should include both hostility and aggressive incidents as outcome measures.

It could be argued that medication alone forms a somewhat restricted medical treatment perspective of a complex phenomenon such as aggression, given the fact that instances of aggression in psychiatric practice are complicated and also have to do with contextual and relational aspects. Moreover, patients are often traumatized and suspicious and many have had experiences of stigma, including from professionals in psychiatry. These issues suggest a range of non-pharmacological interventions that may be studied in reducing aggression in psychiatry. An interesting finding was that childhood trauma was associated with aggressive behaviour. While our findings do not shed light on possible causality, it is entirely plausible that the association with aggressive behaviour is related to unresolved trauma-related schemata impacting perceptions of 
people and the environment. Childhood trauma is often under-diagnosed in patients with psychotic disorders (Grubaugh et al., 2011; Lommen \& Restifo, 2009) and given that the sequelae of trauma are treatable, we offer the hypothesis that treating the sequelae of trauma with psychological therapy will reduce the liability to develop aggressive behaviour. This is in line with previous suggestions for trauma-informed psychosocial interventions (Horowitz et al., 2015) and we feel that this is an interesting hypothesis to test in further research.

\section{Conclusion}

Aggression is a complex and multidimensional concept with many potentially negative consequences for patients, their surroundings and health care workers. This problem cannot be resolved easily using one single approach or solution.

This thesis contributes to the current literature and while more research is needed, there are implications for clinical practice. In the prevention section, we found that there are common warning signs prior to aggressive behaviour in child psychiatry and structured observation tools can be used for clinical risk assessment, but there should be a specific focus on implementation and integration of the instrument within practice. In the epidemiology section, we found low prevalence of aggression. We identified some risk factors associated with aggressive behaviour, of which behavioural symptoms, such as impulsivity, excitement, uncooperativeness and hostility, are of particular concern within clinical practice. We also found childhood trauma to be an important risk factor and recognition and treatment of the sequelae should be considered. Finally, in the treatment section, we concluded that amisulpride could be considered for patients during their first psychotic episode. Clozapine is most effective for the treatment of hostility in persistent psychotic disorders, although efficacy of antipsychotic treatment may depend on the aetiology of aggression.

In addition to the more traditional fields of research, such as risk assessment and pharmacological treatment, there are important changes going on which we believe will change the psychiatric environment. Patients should not be labelled as aggressive or non aggressive but patient-centred interventions, focussed on the relational and contextual factors, should be considered both in future research and in clinical practice. Aggressive behaviour may be inherent to psychiatry and will probably never be completely eliminated. A continued focus on the issue, incorporating research results into practice, could help in a further reduction of incidents, or at least in terms of severity. 



\section{References}




\section{References}

\section{A}

Abderhalden, C., Needham, I., Dassen, T., Halfens, R., Haug, H. J., \& Fischer, J. E. (2008). Structured risk assessment and violence in acute psychiatric wards: randomised controlled trial. Br J Psychiatry, 193(1), 44-50. doi:10.1192/bjp.bp.107.045534

Abderhalden, C., Needham, I., Miserez, B., Almvik, R., Dassen, T., Haug, H. J., \& Fischer, J. E. (2004). Predicting inpatient violence in acute psychiatric wards using the Broset-Violence-Checklist: a multicentre prospective cohort study. J Psychiatr Ment Health Nurs, 11(4), 422-427. doi:10.1111/j.13652850.2004.00733.x

Aleman, A., \& Kahn, R. S. (2001). Effects of the atypical antipsychotic risperidone on hostility and aggression in schizophrenia: a meta-analysis of controlled trials. Eur Neuropsychopharmacol, 11(4), 289-293. doi:10.1016/s0924-977x(01)00097-9

Almvik, R., \& Woods, P. (1999). Predicting inpatient violence using the BrosetViolence Checklist (BVC). Int J Psychiatr Nurs Res, 4(3), 498-505.

Almvik, R., \& Woods, P. (2003). Short-term risk prediction: the Broset Violence Checklist. J Psychiatr Ment Health Nurs, 10(2), 236-238. doi:10.1046/j.1365-2850.2003.00563.x

Amador, X. F., \& Johanson, A.-L. (2000). I am not sick, I don't need help! New York:Vida Press.

American Psychiatric Association. (1994). Diagnostic and Statistical Manual of Mental Health Disorders, 4th edn. Washington DC: American Psychiatric Association.

American Psychiatric Association. (2000). Diagnostic and statistical manual of mental disorders. DSM-IV-TR. Washington DC:American Psychiatric Association.

Amore, M., Menchetti, M., Tonti, C., Scarlatti, F., Lundgren, E., Esposito, W., \& Berardi, D. (2008). Predictors of violent behavior among acute psychiatric patients: clinical study. Psychiatry Clin Neurosci, 62(3), 247-255. doi:10.1111/j.1440-1819.2008.01790.x

Anderson, K. K., \& Jenson, C. E. (2019).Violence risk-assessment screening tools for acute care mental health settings: Literature review. Arch Psychiatr Nurs, 33(1), 112-119. doi:10.1016/j.apnu.2018.08.012

Arango, C. (2000).Violence in schizophrenia. Dialogues Clin Neurosci, 2(4), 392-393.

Arango, C., Bombin, I., Gonzalez-Salvador,T., Garcia-Cabeza, I., \& Bobes, J. (2006). Randomised clinical trial comparing oral versus depot formulations of zuclopenthixol in patients with schizophrenia and previous violence. Eur Psychiatry, 21(1), 34-40. doi:10.1016/j.eurpsy.2005.07.006

Arango, C., Calcedo Barba, A., Gonzalez, S., \& Calcedo Ordonez, A. (1999).Violence in inpatients with schizophrenia: a prospective study. Schizophr Bull, 25(3), 493-503. doi:10.1093/oxfordjournals.schbul. a033396

Arboleda-Florez, J. (2003). Considerations on the stigma of mental illness. Can J Psychiatry, 48(10), 645650. doi:10.1177/070674370304801001 


\section{B}

Baranchik, S., Stryjer, R., Weizman, A., \& Shelef, A. (2019). Add-on benzodiazepines for psychosisinduced aggression. Int Clin Psychopharmacol, 34(3), 119-123. doi:10.1097/YIC.0000000000000254

Barzman, D. H., Brackenbury, L., Sonnier, L., Schnell, B., Cassedy, A., Salisbury, S., . . Mossman, D. (2011). Brief Rating of Aggression by Children and Adolescents (BRACHA): development of a tool for assessing risk of inpatients' aggressive behavior. J Am Acad Psychiatry Law, 39(2), 170-179.

Bernstein, D. P., Fink, L., Handelsman, L., Foote, J., Lovejoy, M., Wenzel, K., . . Ruggiero, J. (1994). Initial reliability and validity of a new retrospective measure of child abuse and neglect. Am J Psychiatry, 151(8), 1132-1136. doi:10.1176/ajp.151.8.1132

Bilezikian. (1998). In J. A. Kennedy (Ed.), Mastering the Kennedy Axis V: A New Psychiatric Assessment of Patient Functioning. Arlington, TX: American Psychiatric Publishing inc.

Bjorkly, S. (1996). Report form for aggressive episodes: preliminary report. Percept Mot Skills, 83(3 Pt 2), 1139-1152. doi:10.2466/pms.1996.83.3f.1139

Blair, R. J. (2016). The Neurobiology of Impulsive Aggression.J Child Adolesc Psychopharmacol, 26(1), 4-9. doi:10.1089/cap.2015.0088

Blake, C. S., \& Hamrin, V. (2007). Current approaches to the assessment and management of anger and aggression in youth: a review. J Child Adolesc Psychiatr Nurs, 20(4), 209-221. doi:10.1111/j.17446171.2007.00102.x

Bland, J. M., \& Altman, D. G. (1986). Statistical methods for assessing agreement between two methods of clinical measurement. Lancet, 1(8476), 307-310.

Blok, G. T., de Beurs, E., de Ranitz, A. G., \& Rinne, T. (2010). [The current psychometric state of risk assessment scales for adults in the Netherlands]. Tijdschr Psychiatr, 52(5), 331-341.

Blyler, C. R., Gold, J. M., Iannone, V. N., \& Buchanan, R. W. (2000). Short form of the WAIS-III for use with patients with schizophrenia. Schizophr Res, 46(2-3), 209-215.

Bobes, J., Fillat, O., \& Arango, C. (2009). Violence among schizophrenia out-patients compliant with medication: prevalence and associated factors. Acta Psychiatr Scand, 119(3), 218-225. doi:10.1111/j.16000447.2008.01302.x

Bonett, D. G. (2002). Sample size requirements for estimating intraclass correlations with desired precision. Stat Med, 21(9), 1331-1335. doi:10.1002/sim.1108

Borenstein, M., Hedges, L., Higgings, J., \& Rothstein, H. (2005). Comprehensive Meta Analysis Version 2. Englewood, NJ: Biostat Inc.

Borenstein, M., Hedges, L., Higgings, T., \& Rothstein, H. (2009). Introduction to Meta-Analysis. Chapter 12. Chichester, UK: Wiley

Borenstein, M., Higgins, J. P., Hedges, L.V., \& Rothstein, H. R. (2017). Basics of meta-analysis: I(2) is not an absolute measure of heterogeneity. Res Synth Methods, 8(1), 5-18. doi:10.1002/jrsm.1230 
Bosqui, T. J., Shannon, C., Tiernan, B., Beattie, N., Ferguson, J., \& Mulholland, C. (2014). Childhood trauma and the risk of violence in adulthood in a population with a psychotic illness.J Psychiatr Res, 54, 121-125. doi:10.1016/j.jpsychires.2014.03.011

Bowers, L. (2014). Safewards: a new model of conflict and containment on psychiatric wards. J Psychiatr Ment Health Nurs, 21(6), 499-508. doi:10.1111/jpm.12129

Bowers, L., Stewart, D., Papadopoulos, C., Dack, C., \& Ross, J. (2011). Inpatient Violence and Aggression: A literature review. Retrieved from:

https://pdfs.semanticscholar.org/b98f/4ff17c264fd919542dcc14905b280c8776b8.pdf

Breier, A., Buchanan, R. W., Kirkpatrick, B., Davis, O. R., Irish, D., Summerfelt, A., \& Carpenter, W. T., Jr. (1994). Effects of clozapine on positive and negative symptoms in outpatients with schizophrenia. Am J Psychiatry, 151(1), 20-26. doi:10.1176/ajp.151.1.20

Brendel, R.W., Wei, M. H., \& Edersheim, J. G. (2010). An approach to the patient in crisis: assessments of the risk of suicide and violence. Med Clin North Am, 94(6), 1089-1102. doi:10.1016/j.mcna.2010.08.002

Brieden,T.,Ujeyl,M., \& Naber,D. (2002).Psychopharmacological treatment of aggression in schizophrenic patients. Pharmacopsychiatry, 35(3), 83-89. doi:10.1055/s-2002-31523

Brosbe, M. S. (2011). Hostile Aggression. In S. Goldstein \& J. A. Naglieri (Eds.), Encyclopedia of Child Behavior and Development (pp. 757-758). Boston, MA: Springer US.

Brucato, G., Appelbaum, P. S., Masucci, M. D., Rolin, S., Wall, M. M., Levin, M., .. Girgis, R. R. (2019). Prevalence and phenomenology of violent ideation and behavior among 200 young people at clinical high-risk for psychosis: an emerging model of violence and psychotic illness. Neuropsychopharmacology, 44(5), 907-914. doi:10.1038/s41386-018-0304-5

Brugman, S., Cornet, L. J., Smeijers, D., Smeets, K., Oostermeijer, S., Buitelaar, J. K., . . Jansen, L. M. (2017). Examining the reactive proactive questionnaire in adults in forensic and non-forensic settings: A variable- and person-based approach. Aggress Behav, 43(2), 155-162. doi:10.1002/ab.21671

Buckley, P. F. (1999). The role of typical and atypical antipsychotic medications in the management of agitation and aggression. J Clin Psychiatry, 60 Suppl 10, 52-60.

Buiter W, Bakker A, R, B.-B., Wubs R, C, v. H., \& M, v. B. (2019). Virtual Reality Training for Professionals to Support Prevention of Patient Aggression in the Workplace. Paper presented at the $11^{\text {th }}$ Violence in Clinial Psychiatry conference.

Bushman, B. J., \& Anderson, C. A. (2001). Is it time to pull the plug on the hostile versus instrumental aggression dichotomy? Psychol Rev, 108(1), 273-279. doi:10.1037/0033-295x.108.1.273

Buss, A. H. (1961). The Psychology of aggression. New York: Wiley.

Cannon-Spoor, H. E., Potkin, S. G., \& Wyatt, R. J. (1982). Measurement of premorbid adjustment in chronic schizophrenia. Schizophr Bull, 8(3), 470-484.

Card, N.A., \& Little, T. D. (2006). Proactive and reactive aggression in childhood and adolescence:A metaanalysis of differential relations with psychosocial adjustment. Int J Behav Dev, 30(5), 466-480. 
Chengappa, K. N., Goldstein, J. M., Greenwood, M., John,V., \& Levine, J. (2003). A post hoc analysis of the impact on hostility and agitation of quetiapine and haloperidol among patients with schizophrenia. Clin Ther, 25(2), 530-541. doi:10.1016/s0149-2918(03)80094-2

Chieze, M., Hurst, S., Kaiser, S., \& Sentissi, O. (2019). Effects of Seclusion and Restraint in Adult Psychiatry: A Systematic Review. Front Psychiatry, 10, 491. doi:10.3389/fpsyt.2019.00491

Choy, O., \& Raine,A. (2018). Omega-3 Supplementation as a Dietary Intervention to Reduce Aggressive and Antisocial Behavior. Curr Psychiatry Rep, 20(5), 32. doi:10.1007/s11920-018-0894-y

Citrome, L., Volavka, J., Czobor, P., Brook, S., Loebel, A., \& Mandel, F. S. (2006). Efficacy of ziprasidone against hostility in schizophrenia: Post hoc analysis of randomized, open-label study data. J Clin Psychiatry, 67(4), 638-642. doi:10.4088/jcp.v67n0415

Citrome, L., Volavka, J., Czobor, P., Sheitman, B., Lindenmayer, J. P., McEvoy, J., . . . Lieberman, J. A. (2001). Effects of clozapine, olanzapine, risperidone, and haloperidol on hostility among patients with schizophrenia. Psychiatr Serv, 52(11), 1510-1514. doi:10.1176/appi.ps.52.11.1510

Claghorn, J., Honigfeld, G., Abuzzahab, F. S., Sr., Wang, R., Steinbook, R., Tuason, V., \& Klerman, G. (1987). The risks and benefits of clozapine versus chlorpromazine. J Clin Psychopharmacol, 7(6), 377-384.

Clarke, D. E., Brown, A. M., \& Griffith, P. (2010). The Broset Violence Checklist: clinical utility in a secure psychiatric intensive care setting. J Psychiatr Ment Health Nurs, 17(7), 614-620. doi:10.1111/j.13652850.2010.01558.x

Coid, J. W., Ullrich, S., Kallis, C., Keers, R., Barker, D., Cowden, F., \& Stamps, R. (2013). The relationship between delusions and violence: findings from the East London first episode psychosis study. $J A M A$ Psychiatry, 70(5), 465-471. doi:10.1001/jamapsychiatry.2013.12

Connor, D. F. (2012). Aggression and antisocial behavior in children and adolescents: Research and treatment. New York: Guilford Press.

Connor, D. F., Newcorn, J. H., Saylor, K. E., Amann, B. H., Scahill, L., Robb, A. S., . . Buitelaar, J. K. (2019). Maladaptive Aggression: With a Focus on Impulsive Aggression in Children and Adolescents. $J$ Child Adolesc Psychopharmacol, 29(8), 576-591. doi:10.1089/cap.2019.0039

Cornell, D. G., Warren, J., Hawk, G., Stafford, E., Oram, G., \& Pine, D. (1996). Psychopathy in instrumental and reactive violent offenders. J Consult Clin Psychol, 64(4), 783-790. doi:10.1037//0022-006x.64.4.783

Correll, C. U., Yu, X., Xiang,Y., Kane, J. M., \& Masand, P. (2017). Biological treatment of acute agitation or aggression with schizophrenia or bipolar disorder in the inpatient setting. Ann Clin Psychiatry, 29(2), 92-107.

Crick, N. R., \& Dodge, K.A. (1996). Social information-processing mechanisms in reactive and proactive aggression. Child Dev, 67(3), 993-1002.

Crocker, J. H., Stargatt, R., \& Denton, C. (2010). Prediction of aggression and restraint in child inpatient units. Aust N Z J Psychiatry, 44(5), 443-449. doi:10.3109/00048670903489825

Czobor, P.,Volavka, J., \& Meibach, R. C. (1995). Effect of risperidone on hostility in schizophrenia.J Clin Psychopharmacol, 15(4), 243-249. doi:10.1097/00004714-199508000-00002 


\section{D}

de Almeida, C. G., Del Grossi Moura, M., Barberato-Filho, S., de Sa Del Fiol, F., Motta, R. H. L., \& de Cassia Bergamaschi, C. (2017). Rapid Tranquilization for Psychiatric Patients with Psychomotor Agitation: What is Known About it? Psychiatr Q, 88(4), 885-895. doi:10.1007/s11126-017-9504-0

De Hert, M., Dirix, N., Demunter, H., \& Correll, C. U. (2011). Prevalence and correlates of seclusion and restraint use in children and adolescents: a systematic review. Eur Child Adolesc Psychiatry, 20(5), 221-230. doi:10.1007/s00787-011-0160-x

de Looff, P., Noordzij, M. L., Moerbeek, M., Nijman, H., Didden, R., \& Embregts, P. (2019). Changes in heart rate and skin conductance in the 30 min preceding aggressive behavior. Psychophysiology, 56(10), e13420. doi:10.1111/psyp.13420

de Ruiter, C. (2007). Wat risicotaxatie wel en niet is. Tijdschrift voor Psychotherapie, 33(2), 87-91.

Dean, A. J., Duke, S. G., Scott, J., Bor, W., George, M., \& McDermott, B. M. (2008). Physical aggression during admission to a child and adolescent inpatient unit: predictors and impact on clinical outcomes. Aust N Z J Psychiatry, 42(6), 536-543. doi:10.1080/00048670802050587

Dean,A.J., Gibbon, P., McDermott, B. M., Davidson,T., \& Scott,J. (2010). Exposure to aggression and the impact on staff in a child and adolescent inpatient unit. Arch Psychiatr Nurs, 24(1), 15-26. doi:10.1016/j. apnu.2009.01.002

Del Vecchio, T. (2011). Instrumental Aggression. In S. Goldstein \& J. A. Naglieri (Eds.), Encyclopedia of Child Behavior and Development (pp. 823-824). Boston, MA: Springer US.

Delcker, A., Schoon, M., Oczkowski, B., \& Gaertner, H. J. P. (1990). Amisulpride versus haloperidol in treatment of schizophrenic patients-results of a double-blind study. Pharmacopsychiatry, 23(03), 125-130. doi:10.1055/s-2007-1014494

Dellazizzo, L., Potvin, S., Bahig, S., \& Dumais, A. (2019). Comprehensive review on virtual reality for the treatment of violence: implications for youth with schizophrenia. NPJ Schizophr, 5(1), 11. doi:10.1038/ s41537-019-0079-7

Desmarais, S. L., Van Dorn, R. A., Johnson, K. L., Grimm, K. J., Douglas, K. S., \& Swartz, M. S. (2014). Community violence perpetration and victimization among adults with mental illnesses. Am J Public Health, 104(12), 2342-2349. doi:10.2105/ajph.2013.301680

Du,M.,Wang, X.,Yin, S., Shu,W.,Hao, R.,Zhao, S.,...Xia,J. (2017). De-escalation techniques for psychosisinduced aggression or agitation. Cochrane Database Syst Rev, 4, Cd009922. doi:10.1002/14651858. CD009922.pub2

Dunlap, W. P., Cortina, J. M., Vaslow, J. B., \& Burke, M. J. (1996). Meta-analysis of experiments with matched groups or repeated measures designs. Psychological methods, 1(2), 170.

Duxbury, J., Bjorkdahl, A., \& Johnson, S. (2006). Ward Culture and Atmosphere. In D. Richter \& R. Whittington (Eds.),Violence in mental health settings. New York: Springer.

Duxbury, J., Hahn, S., Needham, I., \& Pulsford, D. (2008). The Management of Aggression and Violence Attitude Scale (MAVAS): a cross-national comparative study. J Adv Nurs, 62(5), 596-606. doi:10.1111/ j.1365-2648.2008.04629.x 
Duxbury, J., \& Whittington, R. (2005). Causes and management of patient aggression and violence: staff and patient perspectives. J Adv Nurs, 50(5), 469-478. doi:10.1111/j.1365-2648.2005.03426.x

\section{E}

Ebben, C. (2006). Validiteit en betrouwbaarheidsonderzoek naar de Kennedy As V. Retrieved from: http://oaithesis.eur.nl/ir/repub/asset/4111/13944495d68ed3439c78ae5c9a30ca6609155.pdf

Elo, S., \& Kyngas, H. (2008). The qualitative content analysis process. J Adv Nurs, 62(1), 107-115. doi:10.1111/j.1365-2648.2007.04569.x

\section{$\mathbf{F}$}

Faay, M. D. M., van de Sande, R., Gooskens, F., \& Hafsteinsdottir,T. B. (2013). Kennedy Axis V: Clinimetric properties assessed by mental health nurses. Int J Ment Health Nurs, 22(5), 453-464. doi:10.1111/j.14470349.2012.00887.x

Faay, M. D. M.,Valenkamp, M.W., \& Nijman, H. (2017). Warning Signs prior to Aggressive Behavior in Child Psychiatric Units. Arch Psychiatr Nurs, 31(1):43-47. doi:10.1016/j.apnu.2016.07.016

Faay, M. D. M., Czobor, P., \& Sommer, I. E. C. (2018). Efficacy of typical and atypical antipsychotic medication on hostility in patients with psychosis-spectrum disorders: a review and meta-analysis. Neuropsychopharmacology, 43(12), 2340-2349. doi:10.1038/s41386-018-0161-2

Faay, M. D. M., van Os, J., \& Genetic Risk Outcome of Psychosis Investigators. (2020). Aggressive Behavior, Hostility, and Associated Care Needs in Patients With Psychotic Disorders: A 6-Year FollowUp Study. Front Psychiatry, 10, 934. doi: 10.3389/fpsyt.2019.00934

Fazel, S., Gulati, G., Linsell, L., Geddes, J. R., \& Grann, M. (2009). Schizophrenia and violence: systematic review and meta-analysis. PLoS Med, 6(8), e1000120. doi:10.1371/journal.pmed.1000120

Fazel, S., Zetterqvist, J., Larsson, H., Langstrom, N., \& Lichtenstein, P. (2014). Antipsychotics, mood stabilisers, and risk of violent crime. Lancet, 384(9949), 1206-1214. doi:10.1016/s0140-6736(14)60379-2

Finke, L. M. (2001). The use of seclusion is not evidence-based practice. J Child Adolesc Psychiatr Nurs, 14(4), 186-190. doi:10.1111/j.1744-6171.2001.tb00312.x

Fleenor,J.W., Fleenor,J. B., \& Grossnickle,W. F. (1996). Interrater reliability and agreement of performance ratings: A methodological comparison.J Bus and Psychol, 10(3), 367-380. doi: 10.1007/BF02249609

Fluttert, F., Van Meijel, B., Webster, C., Nijman, H., Bartels,A., \& Grypdonck, M. (2008). Risk management by early recognition of warning signs in patients in forensic psychiatric care. Arch Psychiatr Nurs, 22(4), 208-216. doi:10.1016/j.apnu.2007.06.012

Fluttert, F. A.,Van Meijel, B., Bjorkly, S.,Van Leeuwen, M., \& Grypdonck, M. (2013). The investigation of early warning signs of aggression in forensic patients by means of the 'Forensic Early Signs of Aggression Inventory'. J Clin Nurs, 22(11-12), 1550-1558. doi:10.1111/j.1365-2702.2012.04318.x

Foster, C., Bowers, L., \& Nijman, H. (2007). Aggressive behaviour on acute psychiatric wards: prevalence, severity and management. J Adv Nurs, 58(2), 140-149. doi:10.1111/j.1365-2648.2007.04169.x 
Fresán,A., Apiquian, R., de la Fuente-Sandoval, C., Garcia-Anaya, M., Loyzaga, C., \& Nicolini, H. (2004). Premorbid adjustment and violent behavior in schizophrenic patients. Schizophr Res, 69(2-3), 143-148. doi:10.1016/j.schres.2003.07.002

Frogley, C.,Taylor,D.,Dickens, G., \& Picchioni,M.(2012).A systematic review of the evidence of clozapine's anti-aggressive effects. Int J Neuropsychopharmacol, 15(9), 1351-1371. doi:10.1017/S146114571100201X

\section{G}

Gaebel, W., Riesbeck, M., Wolwer, W., Klimke, A., Eickhoff, M., von Wilmsdorff, M., . . Moller, H. J. (2007). Maintenance treatment with risperidone or low-dose haloperidol in first-episode schizophrenia: 1-year results of a randomized controlled trial within the German Research Network on Schizophrenia. J Clin Psychiatry, 68(11), 1763-1774. doi:10.4088/jcp.v68n1116

Gajos, J. M., \& Beaver, K. M. (2016). The effect of omega-3 fatty acids on aggression: A meta-analysis. Neurosci Biobehav Rev, 69, 147-158. doi:10.1016/j.neubiorev.2016.07.017

Gallitano-Mendel, A., Wozniak, D. F., Pehek, E. A., \& Milbrandt, J. (2008). Mice lacking the immediate early gene Egr3 respond to the anti-aggressive effects of clozapine yet are relatively resistant to its sedating effects. Neuropsychopharmacology, 33(6), 1266-1275. doi:10.1038/sj.npp.1301505

Gardner, D. M., Murphy, A. L., O’Donnell, H., Centorrino, F., \& Baldessarini, R. J. (2010). International consensus study of antipsychotic dosing. Am J Psychiatry, 167(6), 686-693. doi:10.1176/appi. ajp.2009.09060802

Gaynes, B. N., Brown, C. L., Lux, L. J., Brownley, K. A., Van Dorn, R. A., Edlund, M. J., . . . Lohr, K. N. (2017). Preventing and De-escalating Aggressive Behavior Among Adult Psychiatric Patients: A Systematic Review of the Evidence. Psychiatr Serv, 68(8), 819-831. doi:10.1176/appi.ps.201600314

Georgieva, I., Vesselinov, R., \& Mulder, C. L. (2012). Early detection of risk factors for seclusion and restraint: a prospective study. Early Interv Psychiatry, 6(4), 415-422. doi:10.1111/j.1751-7893.2011.00330.x

Gibson, L.E.,Alloy, L. B., \& Ellman, L. M. (2016). Trauma and the psychosis spectrum:A review of symptom specificity and explanatory mechanisms. Clin Psychol Rev, 49, 92-105. doi:10.1016/j.cpr.2016.08.003

Gilbert, E., Adams, A., \& Buckingham, C. D. (2011). Examining the relationship between risk assessment and risk management in mental health. J Psychiatr Ment Health Nurs, 18(10), 862-868. doi:10.1111/ j.1365-2850.2011.01737.x

Glazer, W. M., \& Dickson, R. A. (1998). Clozapine reduces violence and persistent aggression in schizophrenia. J Clin Psychiatry, 59 Suppl 3:8-14

Grol, R., \& Grimshaw, J. J. T. 1. (2003). From best evidence to best practice: effective implementation of change in patients' care. Lancet, 362(9391):1225-1230. doi:10.1016/S0140-6736(03)14546-1 362(9391), 1225-1230

Grubaugh, A. L., Zinzow, H. M., Paul, L., Egede, L. E., \& Frueh, B. C. (2011). Trauma exposure and posttraumatic stress disorder in adults with severe mental illness: A critical review. Clin Psychol Rev, 31(6), 883-899. doi:10.1016/j.cpr.2011.04.003 
Gudde, C. B., Olso, T. M., Whittington, R., \& Vatne, S. (2015). Service users' experiences and views of aggressive situations in mental health care: a systematic review and thematic synthesis of qualitative studies.J Multidiscip Healthc, 8, 449-462. doi:10.2147/jmdh.S89486

Guina, J., \& Merrill, B. (2018). Benzodiazepines I: Upping the Care on Downers: The Evidence of Risks, Benefits and Alternatives. J Clin Med, 7(2). doi:10.3390/jcm7020017

Guy, W. (1976). ECDEU assessment manual for psychopharmacology. 534-537. Rockville, MD: US Department of Heath, Education, and Welfare Public Health Service Alcohol, Drug Abuse, and Mental Health Administration

\section{H}

Hage, S., Van Meijel, B., Fluttert, F., \& Berden, G. F. (2009). Aggressive behaviour in adolescent psychiatric settings: what are risk factors, possible interventions and implications for nursing practice? A literature review. J Psychiatr Ment Health Nurs, 16(7), 661-669. doi:10.1111/j.1365-2850.2009.01454.x

Hallett, N., \& Dickens, G. L. (2017). De-escalation of aggressive behaviour in healthcare settings: Concept analysis. Int J Nurs Stud, 75, 10-20. doi:10.1016/j.jinurstu.2017.07.003

Hallett, N., Huber, J.W., Sixsmith, J., \& Dickens, G. L. (2016). Care planning for aggression management in a specialist secure mental health service: An audit of user involvement. Int J Ment Health Nurs, 25(6), 507-515. doi:10.1111/inm.12238

Hankin, C. S., Bronstone, A., \& Koran, L. M. (2011). Agitation in the inpatient psychiatric setting: a review of clinical presentation, burden, and treatment. J Psychiatr Pract, 17(3), 170-185. doi:10.1097/01. pra.0000398410.21374.7d

Harris, A. W., Large, M. M., Redoblado-Hodge, A., Nielssen, O., Anderson, J., Brennan, J. J. A. (2010). Clinical and cognitive associations with aggression in the first episode of psychosis. Aust N ZJ Psychiatry, 44(1), 85-93. doi:10.3109/00048670903270423

Harris, M. R., \& Warren, J. J. (1995). Patient outcomes: assessment issues for the CNS. Clin Nurse Spec, 9(2), 82-86.

Hinkle, D. E., Wiersma, W., \& Jurs, S. G. (1988). Applied statistics for the behavioral sciences. Boston, MA: Houghton Mifflin

Hodgins, S. (2008). Violent behaviour among people with schizophrenia: a framework for investigations of causes, and effective treatment, and prevention. Philos Trans R Soc Lond B Biol Sci, 363(1503), 25052518. doi: $10.1098 /$ rstb.2008.0034

Hodgins, S., \& Klein, S. (2017). New Clinically Relevant Findings about Violence by People with Schizophrenia. Can J Psychiatry, 62(2), 86-93. doi:10.1177/0706743716648300

Hodgins, S., \& Riaz, M. (2011). Violence and phases of illness: differential risk and predictors. Eur Psychiatry, 26(8), 518-524. doi:10.1016/j.eurpsy.2010.09.006

Hoge, R. (2010).Youth level of service/case management inventory. In R. K. Otto \& K. S. Douglas (Eds.), Handbook of Violence Risk Assessment. (pp. 86). New York: Routledge. 
Horowitz, D., Guyer, M., \& Sanders, K. (2015). Psychosocial approaches to violence and aggression: contextually anchored and trauma-informed interventions. CNS Spectr, 20(3), 190-199. doi:10.1017/ s1092852915000280

Huhn, M., Nikolakopoulou, A., Schneider-Thoma, J., Krause, M., Samara, M., Peter, N., ... Leucht, S. (2019). Comparative efficacy and tolerability of 32 oral antipsychotics for the acute treatment of adults with multi-episode schizophrenia: a systematic review and network meta-analysis. Lancet, 394(10202), 939-951. doi:10.1016/s0140-6736(19)31135-3

Husum, T. L., Bjorngaard, J. H., Finset, A., \& Ruud, T. (2010). A cross-sectional prospective study of seclusion, restraint and involuntary medication in acute psychiatric wards: patient, staff and ward characteristics. BMC Health Serv Res, 10, 89. doi:10.1186/1472-6963-10-89

Hvidhjelm,J., Sestoft, D., Skovgaard, L.T., Rasmussen, K.,Almvik, R., \& Bue Bjorner, J. (2016).Aggression in Psychiatric Wards: Effect of the Use of a Structured Risk Assessment. Issues Ment Health Nurs, 37(12), 960-967. doi:10.1080/01612840.2016.1241842

Hyde, S., Fulbrook, P., Fenton, K., \& Kilshaw, M. (2009). A clinical improvement project to develop and implement a decision-making framework for the use of seclusion. Int J Ment Health Nurs, 18(6), 398-408. doi:10.1111/j.1447-0349.2009.00631.x

\section{I}

Inspectorate of Health. (2011). Terugdringen separeren stagneert, normen vereist rondom insluiting psychiatrische patiënten. Uitkomsten inspectieonderzoek naar de preventie van separeren 2008-2011. Inspectorate of Health Retrieved from http://www.igz.nl/Images/2011-12a\%20

Iozzino, L., Ferrari, C., Large, M., Nielssen, O., \& de Girolamo, G. (2015). Prevalence and Risk Factors ofViolence by Psychiatric Acute Inpatients: A Systematic Review and Meta-Analysis. PLoS One, 10(6), e0128536. doi:10.1371/journal.pone.0128536

Isvoranu, A. M., van Borkulo, C. D., Boyette, L. L., Wigman, J.T., Vinkers, C. H., Borsboom, D., \& Group, I. (2017). A Network Approach to Psychosis: Pathways Between Childhood Trauma and Psychotic Symptoms. Schizophr Bull, 43(1), 187-196. doi:10.1093/schbul/sbw055

Itzhaki, M., Bluvstein, I., Peles Bortz, A., Kostistky, H., Bar Noy, D., Filshtinsky, V., \& Theilla, M. (2018). Mental Health Nurse's Exposure to Workplace Violence Leads to Job Stress, Which Leads to Reduced Professional Quality of Life. Front Psychiatry, 9, 59. doi:10.3389/fpsyt.2018.00059

\section{J}

Janssen, W. A., Noorthoorn, E. O., de Vries, W. J., Hutschemeakers, G. J., Lendemeijer, H. H., \& Widdershoven, G. A. (2008). The use of seclusion in the Netherlands compared to countries in and outside Europe. Int J Law Psychiatry, 31(6), 463-470. doi:10.1016/j.ijlp.2008.09.002

Janssen, W. A., van de Sande, R., Noorthoorn, E. O., Nijman, H. L. I., Bowers, L., Mulder, C. L., . . . Steinert, T. (2011). Methodological issues in monitoring the use of coercive measures. Int J Law Psychiatry, 34(6), 429-438. doi:10.1016/j.ijlp.2011.10.008

Jenkins, O., Dye, S., \& Foy, C. (2015). A study of agitation, conflict and containment in association with change in ward physical environment. J Psychiatr Intensive Care, 11(1), 27-35. doi: 10.1017/ S1742646414000065 
Johnson, K. L., Desmarais, S. L., Tueller, S. J., \& Van Dorn, R. A. (2019). Methodological limitations in the measurement and statistical modeling of violence among adults with mental illness. Int J Methods Psychiatr Res, e1776. doi:10.1002/mpr.1776

Kahn, R. S., Winter van Rossum, I., Leucht, S., McGuire, P., Lewis, S. W., Leboyer, M., . . Eijkemans, M. J. C. (2018). Amisulpride and olanzapine followed by open-label treatment with clozapine in first-episode schizophrenia and schizophreniform disorder (OPTiMiSE): a three-phase switching study. Lancet Psychiat, 5(10), 797-807. doi:10.1016/s2215-0366(18)30252-9

Kaltiala-Heino, R., Tuohimaki, C., Korkeila, J., \& Lehtinen, V. (2003). Reasons for using seclusion and restraint in psychiatric inpatient care. Int J Law Psychiatry, 26(2), 139-149. doi:10.1016/s01602527(02)00210-8

Kane,J., Honigfeld, G., Singer,J., \& Meltzer, H. (1988). Clozapine for the treatment-resistant schizophrenic. A double-blind comparison with chlorpromazine. Arch Gen Psychiatry, 45(9), 789-796. doi:10.1001/ archpsyc.1988.01800330013001

Kane, J. M., Marder, S. R., Schooler, N. R., Wirshing, W. C., Umbricht, D., Baker, R. W., . . Borenstein, M. (2001). Clozapine and haloperidol in moderately refractory schizophrenia: a 6-month randomized and double-blind comparison. Arch Gen Psychiatry, 58(10), 965-972. doi:10.1001/archpsyc.58.10.965

Kaunomaki, J., Jokela, M., Kontio, R., Laiho, T., Sailas, E., \& Lindberg, N. (2017). Interventions following a high violence risk assessment score: a naturalistic study on a Finnish psychiatric admission ward. $B M C$ Health Serv Res, 17(1), 26. doi:10.1186/s12913-016-1942-0

Kay, S. R., Fiszbein, A., \& Opler, L. A. (1987). The positive and negative syndrome scale (PANSS) for schizophrenia. Schizophr Bull, 13(2), 261-276. doi:10.1093/schbul/13.2.261

Kelly, T., Simmons, W., \& Gregory, E. (2002). Risk assessment and management: a community forensic mental health practice model. Int J Ment Health Nurs, 11(4), 206-213. doi:10.1046/j.14400979.2002.00250.x

Kennedy, J. A. (2003a). Kennedy Axis V. Retrieved from http://www.kennedymd.com/copyaxisv.html

Kennedy, J. A. (2003b). Mastering the Kennedy Axis V: A New Psychiatric Assessment of Patient Functioning. Arlington, TX:American Psychiatric Publishing inc.

Kennedy, J. A. (2008). Fundamentals of psychiatric treatment planning. Arlington, TX: American Psychiatric Publishing inc.

Keski-Valkama,A., Sailas, E., Eronen, M., Koivisto, A. M., Lonnqvist, J., \& Kaltiala-Heino, R. (2010). The reasons for using restraint and seclusion in psychiatric inpatient care: A nationwide 15-year study. Nord J Psychiatry, 64(2), 136-144. doi:10.3109/08039480903274449

Kinon, B.J., Roychowdhury, S. M., Milton, D. R., \& Hill,A. L. (2001). Effective resolution with olanzapine of acute presentation of behavioral agitation and positive psychotic symptoms in schizophrenia. $J$ Clin Psychiatry, 62 Suppl 2,17-21. 
Klein Tuente, S., Bogaerts, S., van, I. S., \& Veling, W. (2018). Effect of virtual reality aggression prevention training for forensic psychiatric patients (VRAPT): study protocol of a multi-center RCT. BMC Psychiatry, 18(1), 251. doi:10.1186/s12888-018-1830-8

Knezevic,V., Mitrovic, D., Drezgic-Vukic, S., Knezevic, J., Ivezic, A., Siladji-Mladenovic, D., \& Golubovic, B. (2017). Prevalence and Correlates of Aggression and Hostility in Hospitalized Schizophrenic Patients. J Interpers Violence, 32(2), 151-163. doi:10.1177/0886260515585537

Kockler, T. R., Stanford, M. S., Nelson, C. E., Meloy, J. R., \& Sanford, K. (2006). Characterizing aggressive behavior in a forensic population. Am J Orthopsychiatry, 76(1), 80-85. doi:10.1037/0002-9432.76.1.80

Konings, M., Bak, M., Hanssen, M., van Os, J., \& Krabbendam, L. (2006). Validity and reliability of the CAPE: a self-report instrument for the measurement of psychotic experiences in the general population. Acta Psychiatr Scand, 114(1), 55-61. doi:10.1111/j.1600-0447.2005.00741.x

Kontio, R., Anttila, M., Lantta, T., Kauppi, K., Joffe, G., \& Valimaki, M. (2014). Toward a safer working environment on psychiatric wards: service users' delayed perspectives of aggression and violence-related situations and development ideas. Perspect Psychiatr Care, 50(4), 271-279. doi:10.1111/ppc.12054

Kontio, R., Lantta, T., Anttila, M., Kauppi, K., \& Valimaki, M. (2017). Family Involvement in Managing Violence of Mental Health Patients. Perspect Psychiatr Care, 53(1), 55-66. doi:10.1111/ppc.12137

Korver, N., Quee, P. J., Boos, H. B., Simons, C. J., de Haan, L., \& investigators, G. (2012). Genetic Risk and Outcome of Psychosis (GROUP), a multi-site longitudinal cohort study focused on gene-environment interaction: objectives, sample characteristics, recruitment and assessment methods. Int J Methods Psychiatr Res, 21(3), 205-221. doi:10.1002/mpr.1352

Krakowski, M. I., Czobor, P., Citrome, L., Bark, N., \& Cooper, T. B. (2006). Atypical antipsychotic agents in the treatment of violent patients with schizophrenia and schizoaffective disorder. Arch Gen Psychiatry, 63(6), 622-629. doi:10.1001/archpsyc.63.6.622

Krakowski, M. I., Czobor, P., \& Nolan, K. A. (2008). Atypical antipsychotics, neurocognitive deficits, and aggression in schizophrenic patients. J Clin Psychopharmacol, 28(5), 485-493. doi:10.1097/ JCP.0b013e3181855cd6

Kumar, S., \& Simpson, A. I. (2005). Application of risk assessment for violence methods to general adult psychiatry: a selective literature review. Aust N Z J Psychiatry, 39(5), 328-335. doi:10.1080/j.14401614.2005.01579.x

Lahera, G., Herrera, S., Reinares, M., Benito, A., Rullas, M., Gonzalez-Cases, J., \&Vieta, E. (2015). Hostile attributions in bipolar disorder and schizophrenia contribute to poor social functioning. Acta Psychiatr Scand, 131(6), 472-482. doi:10.1111/acps.12399

Landis, J. R., \& Koch, G. G. (1977). The measurement of observer agreement for categorical data. Biometrics, 33(1), 159-174.

Langeveld, J., Bjorkly, S., Auestad, B., Barder, H., Evensen, J., Ten Velden Hegelstad, W., ... Friis, S. (2014). Treatment and violent behavior in persons with first episode psychosis during a 10-year prospective follow-up study. Schizophr Res, 156(2-3), 272-276. doi:10.1016/j.schres.2014.04.010 
Large, M. M., \& Nielssen, O. (2011).Violence in first-episode psychosis: a systematic review and metaanalysis. Schizophr Res, 125(2-3), 209-220. doi:10.1016/j.schres.2010.11.026

Large, M. M., \& Ryan, C. J. (2015).Violence risk assessment has not been shown to reduce violence. Aust N Z J Psychiatry, 49(1), 91. doi:10.1177/0004867414539570

Lee, B. X. (2015). Causes and cures I: Toward a new definition. Aggress Violent Behav, 25, 199-203. doi:https://doi.org/10.1016/j.avb.2015.10.004

Lehoux, C., Gobeil, M.-H., Lefébvre,A.-A., Maziade, M., \& Roy, M.-A. (2009).The Five-Factor Structure of the PANSS: A Critical Review of its Consistency Across Studies. Clin Schizophr Relat Psychoses, 3(2), 103-110. doi:10.3371/csrp.3.2.5

Leucht, S., Cipriani, A., Spineli, L., Mavridis, D., Orey, D., Richter, F, ... Davis, J. M. (2013). Comparative efficacy and tolerability of 15 antipsychotic drugs in schizophrenia: a multiple-treatments meta-analysis. Lancet, 382(9896):951-962. doi:10.1016/S0140-6736(13)60733-3

Leucht, S., Winter-van Rossum, I., Heres, S., Arango, C., Fleischhacker,W.W., Glenthoj, B., . . Sommer, I. E. (2015). The optimization of treatment and management of schizophrenia in Europe (OPTiMiSE) trial: rationale for its methodology and a review of the effectiveness of switching antipsychotics. Schizophr Bull, 41(3), 549-558. doi:10.1093/schbul/sbv019

Lindenmayer, J. P., Bernstein-Hyman, R., \& Grochowski, S. (1994). A new five factor model of schizophrenia. Psychiatr Q, 65(4), 299-322. doi: 10.1007/BF02354306

Ling, S., Umbach, R., \& Raine, A. (2019). Biological explanations of criminal behavior. Psychol Crime Law, 25(6), 626-640. doi:10.1080/1068316x.2019.1572753

Lo, C. H., Tsai, G. E., Liao, C. H., Wang, M.Y., Chang, J. P., Tsuang, H. C., \& Lane, H.Y. (2010). Emotional management and 5-HT2A receptor gene variance in patients with schizophrenia. Biol Psychol, 83(2), 7983. doi:10.1016/j.biopsycho.2009.11.002

Lodewijks, H. P., Doreleijers, T.A., de Ruiter, C., \& Borum, R. (2008). Predictive validity of the Structured Assessment of Violence Risk in Youth (SAVRY) during residential treatment. Int J Law Psychiatry, 31(3), 263-271. doi:10.1016/j.ijlp.2008.04.009

Lommen, M.J.J., \& Restifo, K. (2009). Trauma and Posttraumatic Stress Disorder (PTSD) in Patients with Schizophrenia or Schizoaffective Disorder. Community Ment Health J, 45(6), 485. doi:10.1007/s10597009-9248-x

Lopez-Lopez, I. M., Gomez-Urquiza, J. L., Canadas, G. R., De la Fuente, E. I., Albendin-Garcia, L., \& Canadas-De la Fuente, G. A. (2019). Prevalence of burnout in mental health nurses and related factors: a systematic review and meta-analysis. Int J Ment Health Nurs, 28(5), 1032-1041. doi:10.1111/inm.12606

Lynn, M. R. (1986). Determination and quantification of content validity. Nurs Res, 35(6), 382-385.

\section{M}

Mann-Poll, P. S., Smit, A., Noorthoorn, E. O., Janssen, W. A., Koekkoek, B., \& Hutschemaekers, G. J. M. (2018). Long-Term Impact of a Tailored Seclusion Reduction Program: Evidence for Change? Psychiatr Q, 89(3), 733-746. doi:10.1007/s11126-018-9571-x 
Marder, S. R., Davis, J. M., \& Chouinard, G. (1997). The effects of risperidone on the five dimensions of schizophrenia derived by factor analysis: combined results of the North American trials.J Clin Psychiatry, 58(12), 538-546. doi:10.4088/jcp.v58n1205

Martinez-Martin, N., Fraguas, D., Garcia-Portilla, M. P., Saiz, P. A., Bascaran, M. T., Arango, C., \& Bobes, J. (2011). Self-perceived needs are related to violent behavior among schizophrenia outpatients. J NervMent Dis, 199(9), 666-671. doi:10.1097/NMD.0b013e318229d0d5

McDermott, B. E., \& Holoyda, B. J. (2014). Assessment of aggression in inpatient settings. CNS Spectrums, 19(5), 425-431. doi:10.1017/S1092852914000224

McDowell, M., Nitti, K., Kulstad, E., Cirone, M., Shah, R., Rochford, D., . . Hesse, K. (2019). Clinical Outcomes in Patients Taking Inhaled Loxapine, Haloperidol, or Ziprasidone in the Emergency Department. Clin Neuropharmacol, 42(2), 23-26. doi:10.1097/wnf.0000000000000325

McKenna, B. G., Poole, S. J., Smith, N. A., Coverdale, J. H., \& Gale, C. K. (2003). A survey of threats and violent behaviour by patients against registered nurses in their first year of practice. Int J Ment Health Nurs, 12(1), 56-63. doi:10.1046/j.1440-0979.2003.00269.x

McNiel, D. E., \& Binder, R. L. (1995). Correlates of accuracy in the assessment of psychiatric inpatients' risk of violence. Am J Psychiatry, 152(6), 901-906. doi:10.1176/ajp.152.6.901

Menger, V., Spruit, M., van Est, R., Nap, E., \& Scheepers, F. (2019). Machine Learning Approach to Inpatient Violence Risk Assessment Using Routinely Collected Clinical Notes in Electronic Health Records. JAMA Netw Open, 2(7), e196709. doi:10.1001/jamanetworkopen.2019.6709

Min, S. K., Rhee, C. S., Kim, C. E., \& Kang, D.Y. (1993). Risperidone versus haloperidol in the treatment of chronic schizophrenic patients: a parallel group double-blind comparative trial. Yonsei Med J, 34(2), 179-190. doi:10.3349/ymj.1993.34.2.179

Moher, D., Liberati,A., Tetzlaff, J., \& Altman, D. G. (2009). Preferred reporting items for systematic reviews and meta-analyses: the PRISMA statement. Ann Intern Med, 151(4), 264-269, w264. doi:10.7326/00034819-151-4-200908180-00135

Montoya, A., Valladares, A., Lizán, L., San, L., Escobar, R., Paz, S. J. H., \& Outcomes, Q. o. L. (2011). Validation of the Excited Component of the Positive and Negative Syndrome Scale (PANSS-EC) in a naturalistic sample of 278 patients with acute psychosis and agitation in a psychiatric emergency room. Health Qual Life Out,9:18. doi:10.1186/1477-7525-9-18

Morosini, P. L., Magliano, L., Brambilla, L., Ugolini, S., \& Pioli, R. (2000). Development, reliability and acceptability of a new version of the DSM-IV Social and Occupational Functioning Assessment Scale (SOFAS) to assess routine social funtioning. Acta Psychiatr Scand, 101(4), 323-329. doi:10.1034/j.16000447.2000.101004323.x

Morrison, E. F. (1990).Violent psychiatric inpatients in a public hospital. Sch Inq Nurs Pract, 4(1), 65-82; discussion 83-66.

Morse, J. M., \& Field, P.A. (1995). Nursing research: The application of qualitative approaches. Nelson Thornes. 
Moulin,V., Baumann, P., Gholamrezaee, M., Alameda, L., Palix, J., Gasser, J., \& Conus, P. (2018). Cannabis, a Significant Risk Factor for Violent Behavior in the Early Phase Psychosis. Two Patterns of Interaction of Factors Increase the Risk ofViolent Behavior: Cannabis Use Disorder and Impulsivity; Cannabis Use Disorder, Lack of Insight and Treatment Adherence. Front Psychiatry, 9, 294. doi:10.3389/fpsyt.2018.00294

Moulin, V., Golay, P., Palix, J., Baumann, P. S., Gholamrezaee, M. M., Azzola, A., . . . Conus, P. (2018). Impulsivity in early psychosis: A complex link with violent behaviour and a target for intervention. Eur Psychiatry, 49, 30-36. doi:10.1016/j.eurpsy.2017.12.003

Muir-Cochrane, E., Oster, C., Gerace, A., Dawson, S., Damarell, R., \& Grimmer, K. (2019). The effectiveness of chemical restraint in managing acute agitation and aggression: A systematic review of randomized controlled trials. Int J Ment Health Nurs, 29(2):110-126. doi:10.1111/inm.12654

Mulder, C. L. (2000). Kennedy As V: Vertaling En Bewerking. Rotterdam: Erasmus Universiteit.

Mundo, E., Bonalume, L., Del Corno, F., Madeddu, F., \& Lang, M. (2010). [Kennedy V Axis assessment in an Italian outpatient and inpatient population]. Riv Psichiatr, 45(4), 214-220.

National Collaborating Centre for Mental Health. (2015). National Institute for Health and Care Excellence: Clinical Guidelines. Violence and Aggression: Short-Term Management in Mental Health, Health and Community Settings: Updated edition. London:The British Psychological Society \& The Royal College of Psychiatrists.

National Institute for Clinical Excellence. (2005). Violence: The short-term management of disturbed/violent behaviour in psychiatric in-patient settings and emergency departments. Retrieved from:

http://guidance.nice.org.uk/CG25/NICEGuidance/pdf/English

Newson, R. B. (2013). Attributable and unattributable risks and fractions and other scenario comparisons. Stata Journal, 13(4), 672-698. Retrieved from: http://www.stata-journal.com/article.html?article =st0314

Nieuwlaat, R., Wilczynski, N., Navarro, T., Hobson, N., Jeffery, R., Keepanasseril, A., . . . et al. (2014). Interventions for enhancing medication adherence. Cochrane Database Syst Rev, (11). doi:10.1002/14651858.CD000011.pub4

Nijman, H., \& Palmstierna, T. (2002). Measuring aggression with the staff observation aggression scale-revised. Acta Psychiatr Scand Suppl, (412), 101-102. doi:10.1034/j.1600-0447.106.s412.21.x

Nijman, H. L. (2002). A model of aggression in psychiatric hospitals. Acta Psychiatr Scand Suppl, (412), 142-143. doi:10.1034/j.1600-0447.106.s412.30.x

Nijman, H. L., aCampo, J. M., Ravelli, D. P., \& Merckelbach, H. L. (1999).A tentative model of aggression on inpatient psychiatric wards. Psychiatr Serv, 50(6), 832-834. doi:10.1176/ps.50.6.832

Nijman, H. L., Allertz, W. F., Merckelbach, H., A Campo, J., \& Ravelli, D. (1997).Aggressive behaviour on an acute psychiatric admissions ward. Eur J Psychiatry, 11(2), 106-114. doi:10.1176/ps.48.5.694

Nijman, H. L. I., Muris, P., Merckelbach, H. L. G. J., Palmstierna, T., Wistedt, B., Vos, A. M., . . . Allertz, W. (1999). The staff observation aggression scale-revised (SOAS-R). Aggress Behav, 25(3), $197-209$. doi:10.1002/(sici)1098-2337(1999)25:3<197::Aid-ab4>3.0.Co;2-c 
Noorthoorn, E., Lepping, P., Janssen, W., Hoogendoorn, A., Nijman, H., Widdershoven, G., \& Steinert, T. (2015). One-year incidence and prevalence of seclusion: Dutch findings in an international perspective. Soc Psychiatry Psychiatr Epidemiol, 50(12), 1857-1869. doi:10.1007/s00127-015-1094-2

Noorthoorn, E., Voskes,Y., Janssen,W.A., Mulder, C. L., van de Sande, R., Nijman, H. L., ... Widdershoven, G. A. (2016). Seclusion Reduction in Dutch Mental Health Care: Did Hospitals Meet Goals? Psychiatr Serv, 67(12), 1321-1327. doi:10.1176/appi.ps.201500414

Oakley, C., Harris, S., Fahy, T., Murphy, D., \& Picchioni, M. (2016). Childhood adversity and conduct disorder: A developmental pathway to violence in schizophrenia. Schizophr Res, 172(1-3), 54-59. doi:10.1016/j.schres.2016.01.047

Odintsova, V. V., Roetman, P. J., Ip, H. F., Pool, R., Van der Laan, C. M., Tona, K. D., . . . Boomsma, D. I. (2019). Genomics of human aggression: current state of genome-wide studies and an automated systematic review tool. Psychiatr Genet, 29(5), 170-190. doi:10.1097/ypg.0000000000000239

Ose, S. O., Lilleeng, S., Pettersen, I., Ruud, T., \& van Weeghel, J. (2017). Risk of violence among patients in psychiatric treatment: results from a national census. Nord J Psychiatry, 71(8), 551-560. doi:10.1080/0 8039488.2017.1352024

Otto, R. K., \& Douglas, K. S. (2011). Handbook of violence risk assessment. New York: Routledge.

Overall, J. E., \& Gorham, D. R. (1962). The Brief Psychiatric Rating Scale. Psychological Reports, 10(3), 799-812. doi:10.2466/pr0.1962.10.3.799

Oxford Dictionary \& Thesaurus. (2010a). “aggression”: Oxford University Press

Oxford Dictionary \& Thesaurus. (2010b). "hostility": Oxford University Press

Oxford English Dictionary. (2019a). "aggression”. Retrieved from:

https://www.oed.com/view/Entry/3951?redirectedFrom=aggression

Oxford English Dictionary. (2019b). "hostility". Retrieved from:

https://www.oed.com/view/Entry/88772?redirectedFrom=hostility

Oxford English Dictionary. (2019c). "violence". Retrieved from:

https://www.oed.com/view/Entry/223638?rskey=0JOg9H\&amp;result=1\&amp;isAdvanced=false

\section{P}

Pacciardi, B., Calcedo, A., \& Messer, T. (2019). Inhaled Loxapine for the Management of Acute Agitation in Bipolar Disorder and Schizophrenia: Expert Review and Commentary in an Era of Change. Drugs $R$ D, 19(1), 15-25. doi:10.1007/s40268-019-0262-3

Paillot, C. M. (2009). Double blind, randomized, controlled study of a psychotherapy designed to improve motivation for change, insight into schizophrenia and adherence to medication. Schizophrenia Bulletin 35 , (suppl 1), 343.

Panagiotou, A., Mafreda, C., Moustikiadis, A., \& Prezerakos, P. (2019). Modifiable factors affecting inpatient violence in an acute child and adolescent psychiatric unit: A 16-year retrospective study. Int $J$ Ment Health Nurs, 28(5), 1078-1089. doi:10.1111/inm.12619 
Papadopoulos, C., Ross, J., Stewart, D., Dack, C., James, K., \& Bowers, L. (2012). The antecedents of violence and aggression within psychiatric in-patient settings. Acta Psychiatr Scand, 125(6), 425-439. doi:10.1111/j.1600-0447.2012.01827.x

Peuskens, J. (1995). Risperidone in the treatment of patients with chronic schizophrenia: a multi-national, multi-centre, double-blind, parallel-group study versus haloperidol. Risperidone Study Group. $\mathrm{Br} J$ Psychiatry, 166(6), 712-726; discussion 727-733. doi:10.1192/bjp.166.6.712

Phelan, M., Slade, M., Thornicroft, G., Dunn, G., Holloway, F., Wykes, T., . . Hayward, P. (1995). The Camberwell Assessment of Need: the validity and reliability of an instrument to assess the needs of people with severe mental illness. Br J Psychiatry, 167(5), 589-595. doi:10.1192/bjp.167.5.589

Phillips, N. L., Stargatt, R., \& Fisher, L. (2011). Risk assessment: predicting physical aggression in child psychiatric inpatient units. Aust N ZJ Psychiatry, 45(8), 638-645. doi:10.3109/00048674.2011.587396

Polit, D. F., \& Beck, C. T. (2008). Nursing research: Generating and assessing evidence for nursing practice. Philadelphia US: Lippincott Williams \& Wilkins.

Polit, D. F., \& Lake, E. (2010). Statistics and data analysis for nursing research (Vol. 1). Upper Saddle River, NJ: Pearson.

Price, O., Baker, J., Bee, P., Grundy, A., Scott, A., Butler, D., . . Lovell, K. (2018). Patient perspectives on barriers and enablers to the use and effectiveness of de-escalation techniques for the management of violence and aggression in mental health settings. J Adv Nurs, 74(3), 614-625. doi:10.1111/jan.13488

Price, O., Baker, J., Bee, P., \& Lovell, K. (2015). Learning and performance outcomes of mental health staff training in de-escalation techniques for the management of violence and aggression. Br J Psychiatry, 206(6), 447-455. doi:10.1192/bjp.bp.114.144576

\section{$\mathbf{R}$}

Reebye, P. (2005). Aggression during early years - infancy and preschool. Can Child Adolesc Psychiatr Rev, 14(1), 16-20.

Richter, D., \& Whittington, R. (2006). Towards an evidence-based approach of violence in mental health settings. In D. Richter \& R. Whittington (Eds.). Violence in mental health settings. New York: Springer.

Ryan, E. P., Hart, V. S., Messick, D. L., Aaron, J., \& Burnette, M. (2004). A prospective study of assault against staff by youths in a state psychiatric hospital. Psychiatr Serv, 55(6), 665-670. doi:10.1176/appi. ps.55.6.665

\section{S}

Sailas, E. E. S., \& Fenton, M. (2000). Seclusion and restraint for people with serious mental illnesses. Cochrane Database Syst Rev, (1). doi:10.1002/14651858.CD001163

Sarver, W. L., Radziewicz, R., Coyne, G., Colon, K., \& Mantz, L. (2019). Implementation of the Broset Violence Checklist on an Acute Psychiatric Unit. J Am Psychiatr Nurses Assoc, 25(6), 476-486. doi:10.1177/1078390318820668

Schaftenaar, P., van Outheusden, I., Stams, G. J., \& Baart, A. (2018). Relational caring and contact after treatment. An evaluation study on criminal recidivism. Int J Law Psychiatry, 60, 45-50. doi:10.1016/j. ijlp.2018.07.011 
Sequeira, H. (2004). The psychological effects on nursing staff of administering physical restraint in a secure psychiatric hospital: 'When I go home, it's then that I think about it'. Br J Forensic Pract, 6(1), 3-15. doi:10.1108/14636646200400002

Sheehan, D.V., Lecrubier,Y., Sheehan, K. H., Amorim, P., Janavs, J., Weiller, E., .. Dunbar, G. C. (1998).The Mini-International Neuropsychiatric Interview (MINI): the development and validation of a structured diagnostic psychiatric interview for DSM-IV and ICD-10. J Clin Psychiatry, 59 Suppl 20:22-33

Shrout, P. E., \& Fleiss, J. L. (1979). Intraclass correlations: uses in assessing rater reliability. Psychol Bull, 86(2), 420-428. doi:10.1037//0033-2909.86.2.420

Silver, H. (2001). Fluvoxamine as an adjunctive agent in schizophrenia. CNS Drug Rev, 7(3), $283-304$. doi:10.1111/j.1527-3458.2001.tb00200.x

Slade, M., Beck, A., Bindman, J., Thornicroft, G., \& Wright, S. (1999). Routine clinical outcome measures for patients with severe mental illness: CANSAS and HoNOS. Br J Psychiatry, 174, 404-408. doi:10.1192/ bjp.174.5.404

Smeets, K. C., Oostermeijer, S., Lappenschaar, M., Cohn, M., van der Meer, J. M., Popma, A., ... Buitelaar, J. K. (2017). Are Proactive and Reactive Aggression Meaningful Distinctions in Adolescents? A Variableand Person-Based Approach. J Abnorm Child Psychol, 45(1), 1-14. doi:10.1007/s10802-016-0149-5

Spivak, B., Mester, R., Wittenberg, N., Maman, Z., \& Weizman, A. (1997). Reduction of aggressiveness and impulsiveness during clozapine treatment in chronic neuroleptic-resistant schizophrenic patients. Clin Neuropharmacol, 20(5), 442-446. doi:10.1097/00002826-199710000-00009

SPSS Inc. (2007). SPSS for Windows Version 16.0. Chicago, SPSS Inc.

Stahl, S. M. (2014). Deconstructing violence as a medical syndrome: mapping psychotic, impulsive, and predatory subtypes to malfunctioning brain circuits. CNS Spectr, 19(5), 357-365. doi:10.1017/ s1092852914000522

StataCorp. (2017). Stata Statistical Software: Release 15.

Steinert, T. (2006). Prediction of aggression in inpatient settings. In D. Richter \& R. Whittington (Eds.), Violence in mental health settings. New York: Springer.

Steinert, T., Lepping, P., Bernhardsgrutter, R., Conca, A., Hatling, T., Janssen, W., . . . Whittington, R. (2010). Incidence of seclusion and restraint in psychiatric hospitals: a literature review and survey of international trends. Soc Psychiatry Psychiatr Epidemiol, 45(9), 889-897. doi:10.1007/s00127-009-0132-3

Sukhodolsky, D. G., Cardona, L., \& Martin, A. (2005). Characterizing Aggressive and Noncompliant Behaviors in a Children's Psychiatric Inpatient Setting. Child Psychiatry Hum Dev, 36(2), $177-193$. doi:10.1007/s10578-005-3494-0

Sutradhar, R., \& Austin, P. C. (2018). Relative rates not relative risks: addressing a widespread misinterpretation of hazard ratios. Ann Epidemiol, 28(1), 54-57. doi:10.1016/j.annepidem.2017.10.014

Swanson, J. W., Swartz, M. S., \& Elbogen, E. B. (2004). Effectiveness of atypical antipsychotic medications in reducing violent behavior among persons with schizophrenia in community-based treatment. Schizophr Bull, 30(1), 3-20. doi:10.1093/oxfordjournals.schbul.a007065 
Swanson, J. W., Swartz, M. S., Van Dorn, R. A., Elbogen, E. B., Wagner, H. R., Rosenheck, R. A., . . . Lieberman, J. A. (2006). A national study of violent behavior in persons with schizophrenia. Arch Gen Psychiatry, 63(5), 490-499. doi:10.1001/archpsyc.63.5.490

Swanson, J. W., Swartz, M. S., Van Dorn, R. A., Volavka, J., Monahan, J., Stroup, T. S., . . investigators, C. (2008). Comparison of antipsychotic medication effects on reducing violence in people with schizophrenia. Br J Psychiatry, 193(1), 37-43. doi:10.1192/bjp.bp.107.042630

\section{T}

Taylor, P. J. (2008). Psychosis and violence: stories, fears, and reality. Can J Psychiatry, 53(10), 647-659. doi:10.1177/070674370805301004

Teplin, L.A., McClelland, G. M.,Abram, K. M., \&Weiner, D.A. (2005). Crime victimization in adults with severe mental illness: comparison with the National Crime Victimization Survey. Arch Gen Psychiatry, 62(8), 911-921. doi:10.1001/archpsyc.62.8.911

Terkelsen, T. B., \& Larsen, I. B. (2016). Fear, danger and aggression in a Norwegian locked psychiatric ward: Dialogue and ethics of care as contributions to combating difficult situations. Nurs Ethics, 23(3), 308-317. doi:10.1177/0969733014564104

Terwee, C. B., Bot, S. D., de Boer, M. R., van der Windt, D. A., Knol, D. L., Dekker, J., ... de Vet, H. C. (2007). Quality criteria were proposed for measurement properties of health status questionnaires. J Clin Epidemiol, 60(1), 34-42. doi:10.1016/j.jclinepi.2006.03.012

Topiwala, A., \& Fazel, S. (2011). The pharmacological management of violence in schizophrenia: a structured review. Expert Rev Neurother, 11(1), 53-63. doi:10.1586/ern.10.180

Torrey, E. F. (2011). Stigma and violence: isn't it time to connect the dots? Schizophr bull, 37(5), 892-896. doi:10.1093/schbul/sbr057

Tunde-Ayinmode, M., \& Little, J. (2004). Use of seclusion in a psychiatric acute inpatient unit. Australas Psychiatry, 12(4), 347-351. doi:10.1080/j.1440-1665.2004.02125.x

\section{U}

Ulrich, R. S., Bogren, L., Gardiner, S. K., \& Lundin, S. (2018). Psychiatric ward design can reduce aggressive behavior. Journal Environ Psychol, 57, 53-66. doi:10.1016/j.jenvp.2018.05.002

\section{V}

Valenkamp, M. W. (2011). Preventing the use of restrictive interventions. Rotterdam: Erasmus Universiteit

Valenkamp, M. W., Verheij, F., Nijman, H., Palmstierna, T., \& Bjorkly, S. (2013). Proactive monitoring of aggression in children tool. Rotterdam: afdeling kinder- en jeugdpsychiatrie Erasmus MC-Sophia.

van de Sande, R. (2007). Het beoordelen en bewaken van acute gezondheidsbedreigende risico's. In M. Jansen, M. Kuiper, J. Ettema, \& R. van de Sande (Eds.), De Expertverpleegkundige: Houten: Bohn Stafleu van Loghum.

van de Sande, R., Nijman, H. L., Noorthoorn, E. O., Wierdsma, A. I., Hellendoorn, E., van der Staak, C., \& Mulder, C. L. (2011). Aggression and seclusion on acute psychiatric wards: effect of short-term risk assessment. Br J Psychiatry, 199(6), 473-478. doi:10.1192/bjp.bp.111.095141 
van de Sande, R., Noorthoorn, E., Nijman, H., Wierdsma, A., van de Staak, C., Hellendoorn, E., \& Mulder, N. (2017). Associations between psychiatric symptoms and seclusion use: Clinical implications for care planning. Int J Ment Health Nurs, 26(5), 423-436. doi:10.1111/inm.12381

van de Sande, R., Noorthoorn, E., Wierdsma, A., Hellendoorn, E., van der Staak, C., Mulder, C. L., \& Nijman, H. (2013). Association between short-term structured risk assessment outcomes and seclusion. Int J Ment Health Nurs, 22(6), 475-484. doi:10.1111/inm.12033

van der Schaaf, P. S., Dusseldorp, E., Keuning, F. M., Janssen, W. A., \& Noorthoorn, E. O. (2013). Impact of the physical environment of psychiatric wards on the use of seclusion. Br J Psychiatry, 202, $142-149$. doi:10.1192/bjp.bp.112.118422

van Kessel, K., Milne, D., Hunt, K., \& Reed, P. W. (2012). Understanding inpatient violence in a New Zealand child and adolescent psychiatric setting. Int J Ment Health Nurs, 21(4), 320-329. doi:10.1111/ j.1447-0349.2011.00789.x

van Melle, A. L., Voskes, Y., de Vet, H. C. W., van der Meijs, J., Mulder, C. L., \& Widdershoven, G. A. M. (2019). High and Intensive Care in Psychiatry: Validating the HIC Monitor as a Tool for Assessing the Quality of Psychiatric Intensive Care Units. Adm Policy Ment Health, 46(1), 34-43. doi:10.1007/s10488018-0890-x

van Mierlo, T., Bovenberg, F., Voskes, Y., \& Mulder, N. (2013). Werkboek HIC. High en intensive care in de psychiatrie. Utrecht: De Tijdstroom.

van Os, J., Marsman, A., van Dam, D., Simons, C. J., \& Investigators, G. (2017). Evidence That the Impact of Childhood Trauma on IQ Is Substantial in Controls, Moderate in Siblings, and Absent in Patients With Psychotic Disorder. Schizophr Bull, 43(2), 316-324. doi:10.1093/schbul/sbw177

Varshney, M., Mahapatra, A., Krishnan, V., Gupta, R., \& Deb, K. S. (2016). Violence and mental illness: what is the true story? J Epidemiol Community Health, 70(3):223-225. doi:10.1136/jech-2015-205546

Velthorst, E., Levine, S. Z., Henquet, C., de Haan, L., van Os, J., Myin-Germeys, I., \& Reichenberg, A. (2013). To cut a short test even shorter: reliability and validity of a brief assessment of intellectual ability in schizophrenia--a control-case family study. Cogn Neuropsychiatry, 18(6), 574-593. doi:10.1080/13546 805.2012 .731390

Vermeulen, J. M., Doedens, P., Boyette, L. N. J., Spek, B., Latour, C. H. M., \& de Haan, L. (2019). “But I did not touch nobody!'-Patients' and nurses' perspectives and recommendations after aggression on psychiatric wards-A qualitative study. J Adv Nurs, 75(11): 2845-2854. doi:10.1111/jan.14107

Volavka, J. (2008). Neurobiology of violence. Washington, DC:American Psychiatric Pub

Volavka, J., \& Citrome, L. (2008). Heterogeneity of violence in schizophrenia and implications for longterm treatment. Int J Clin Pract, 62(8), 1237-1245. doi:10.1111/j.1742-1241.2008.01797.x

Volavka, J., \& Citrome, L. (2011). Pathways to aggression in schizophrenia affect results of treatment. Schizophr Bull, 37(5), 921-929. doi:10.1093/schbul/sbr041

Volavka, J., \& Citrome, L. (2018). Psychopharmacology of violence. In A.T.Vazsonyi, D. J. Flannary, \& M. DeLisi (Eds.), The Cambridge Handbook of Violent Behavior and Aggression. Cambridge, UK: Cambrigde University Press. 
Volavka, J., Czobor, P., Citrome, L., \&Van Dorn, R.A. (2014). Effectiveness of antipsychotic drugs against hostility in patients with schizophrenia in the Clinical Antipsychotic Trials of Intervention Effectiveness (CATIE) study. CNS Spectr, 19(5), 374-381. doi:10.1017/S1092852913000849

Volavka, J., Czobor, P., Derks, E. M., Bitter, I., Libiger, J., Kahn, R. S., . . Group, E. S. (2011). Efficacy of antipsychotic drugs against hostility in the European First-Episode Schizophrenia Trial (EUFEST).J Clin Psychiatry, 72(7), 955-961. doi:10.4088/JCP.10m06529

Volavka, J., Czobor, P., Nolan, K., Sheitman, B., Lindenmayer, J. P., Citrome, L., . . . Lieberman, J. A. (2004). Overt aggression and psychotic symptoms in patients with schizophrenia treated with clozapine, olanzapine, risperidone, or haloperidol. J Clin Psychopharmacol, 24(2), 225-228. doi:10.1097/01. jcp.0000117424.05703.29

Volavka, J., Van Dorn, R.A., Citrome, L., Kahn, R. S., Fleischhacker, W.W., \& Czobor, P. (2016). Hostility in schizophrenia: An integrated analysis of the combined Clinical Antipsychotic Trials of Intervention Effectiveness (CATIE) and the European First Episode Schizophrenia Trial (EUFEST) studies. Eur Psychiatry, 31, 13-19. doi:10.1016/j.eurpsy.2015.10.003

Voskes, Y., Kemper, M., Landeweer, E. G., \& Widdershoven, G. A. (2014). Preventing seclusion in psychiatry: a care ethics perspective on the first five minutes at admission. Nurs Ethics, 21(7), 766-773. doi:10.1177/0969733013493217

\section{W}

Warnez, S., \& Alessi-Severini, S. (2014). Clozapine: a review of clinical practice guidelines and prescribing trends. BMC Psychiatry, 14, 102. doi:10.1186/1471-244x-14-102

Watts, D., Bindman, J., Slade, M., Holloway, F., Rosen, A., \& Thornicroft, G. (2004). Clinical assessment of risk decision support (CARDS): The development and evaluation of a feasible violence risk assessment for routine psychiatric practice. J Ment Health, 13(6), 569-581. doi:10.1080/09638230400017087

Webster, C. D., Douglas, K. S., Eaves, D., \& Hart, S. (1997). HCR-20: Assessing the Risk for Violence. (Version 2). Vancouver: Mental Health, Law, and Policy Institute, Simon Fraser University.

Webster, C. D., \& Hucker, S. J. (2007). Violence risk assessment and management. Chichester, UK: Wiley.

Webster, C. D., Hucker, S. J., \& Bloom, H. (2002). Transcending the actuarial versus clinical polemic in assessing risk for violence. Criminal justice and behavior, 29(5), 659-665. doi:10.1177/009385402236736

Welleman, R., Stringer, B., Landeweer, E., \& Gijsbers van Wijk, C. M.T. (2011). De eerste viif minuten in de verlenging. Implementatie en borging van best practices dwangreductie (2008-2011). Amsterdam: GGZ inGeest.

Whiting, D., Lennox, B. R., \& Fazel, S. (2019). Violent outcomes in first-episode psychosis: A clinical cohort study. Early Interv Psychiatry. doi:10.1111/eip.12901

Whiting, P., Rutjes, A.W. S., Dinnes, J., Reitsma, J. B., Bossuyt, P. M. M., \& Kleijnen, J. (2004). Development and validation of methods for assessing the quality of diagnostic accuracy studies. Health Technol Assess, 8(25):iii-234. doi:10.3310/hta8250

Whitley, G. G., Jacobson, G. A., \& Gawrys, M.T. (1996). The impact of violence in the health care setting upon nursing education. J Nurs Educ, 35(5), 211-218. 
Winsper, C., Ganapathy, R., Marwaha, S., Large, M., Birchwood, M., \& Singh, S. P. (2013). A systematic review and meta-regression analysis of aggression during the first episode of psychosis. Acta Psychiatr Scand, 128(6), 413-421. doi:10.1111/acps.12113

Witt, K., Hawton, K., \& Fazel, S. (2014). The relationship between suicide and violence in schizophrenia: analysis of the Clinical Antipsychotic Trials of Intervention Effectiveness (CATIE) dataset. Schizophr Res, 154(1-3), 61-67. doi:10.1016/j.schres.2014.02.001

Witt, K., van Dorn, R., \& Fazel, S. (2013). Risk factors for violence in psychosis: systematic review and meta-regression analysis of 110 studies. PLoS One, 8(2), e55942. doi:10.1371/journal.pone.0055942

Woods, P., \& Almvik, R. (2002). The Broset violence checklist (BVC). Acta Psychiatr Scand Suppl(412), 103-105. doi:10.1034/j.1600-0447.106.s412.22.x

Woods, P., \& Ashley, C. (2007).Violence and aggression: a literature review. J Psychiatr Ment Health Nurs, 14(7), 652-660. doi:10.1111/j.1365-2850.2007.01149.x

World Health Organization. (1996). Global Consultation on Violence and Health. Violence: a public health priority. Retrieved from:

https://www.who.int/violence_injury_prevention/violence/world_report/en/full_en.pdf

World Health Organization (WHO). (1990). Composite International Diagnostic Interview (CIDI): a) CIDIinterview (version 1.0), b) CIDI-user manual, c) CIDI-training manual, d) CIDI-computer programs, Geneva, WHO.

\section{$Y$}

Yang, B. X., Stone, T. E., Petrini, M.A., \& Morris, D. L. (2018). Incidence, Type, Related Factors, and Effect of Workplace Violence on Mental Health Nurses: A Cross-sectional Survey. Arch Psychiatr Nurs, 32(1), 3138. doi:10.1016/j.apnu.2017.09.013

\section{Z}

Zaman, H., Sampson, S., Beck, A., Sharma, T., Clay, F., Spyridi, S., . . Gillies, D. (2018). Benzodiazepines for Psychosis-Induced Aggression or Agitation. Schizophr Bull, 44(5), 966-969. doi:10.1093/schbul/sby056 

Samenvatting

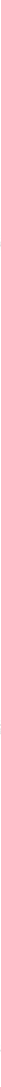





\section{Samenvatting}

Agressie is een groot probleem binnen de psychiatrie met nadelige gevolgen voor patiënten, hun omgeving en professionals. Er is de afgelopen jaren een toename geweest in onderzoek naar agressie, maar desondanks zijn veel klinische beslissingen niet evidence-based.

Het doel van deze dissertatie is om een bijdrage te leveren aan de huidige kennis over agressie in de psychiatrie met klinisch relevante resultaten. Het gaat hierbij om drie thema's: preventie, epidemiologie en behandeling. Deze drie thema's zijn besproken in de vijf onderzoeken van deze dissertatie (hoofdstuk $2 \mathrm{t} / \mathrm{m} 6$ ). Hieronder volgt een beknopte samenvatting van deze vijf hoofdstukken, gevolgd door een algemene conclusie.

\section{Waarschuwingssignalen voorafgaand aan agressie in de kinderpsychiatrie}

In hoofdstuk 2 keken we naar de gedragsveranderingen bij kinderen, voorafgaand aan agressie. Waar er bij volwassenen onderzoek is gedaan naar deze zogenoemde waarschuwingssignalen, is hier bij kinderen nog onvoldoende over bekend. Kennis van deze waarschuwingssignalen zorgt ervoor dat professionals agressie eerder herkennen en preventieve interventies kunnen inzetten om agressie te voorkomen.

Het doelvan ditonderzoek is om de meest voorkomende waarschuwingssignalen voorafgaand aan agressie bij kinderen te identificeren. We gebruikten hiervoor meldingsformulieren die medewerkers invullen na een agressie incident. Deze gegevens waren reeds verzameld in het kader van een ander onderzoek. De formulieren werden ingevuld op psychiatrische (dag)klinische afdelingen binnen diverse instellingen voor kinderen van 4 tot 12 jaar oud. We gebruikten de antwoorden van één specifieke vraag op het meldingsformulier: "welke waarschuwingssignalen hebt $\mathrm{u}$ waargenomen (benoem in steekwoorden)?" Dit formulier heeft tevens een maat voor de ernst van het incident: milde incidenten (zonder fysieke gevolgen) of ernstiger incidenten (met fysieke gevolgen).

In totaal zijn er 1068 meldingsformulieren ingevuld. Op 575 (53.8\%) meldingsformulieren werd één of meer waarschuwingssignaal beschreven. Middels een coderingsproces werden deze open antwoorden geclusterd om tot vergelijkbare termen te komen. Dit coderingsproces resulteerde in 16 verschillende signalen. De drie meest voorkomende signalen waren "onrust," "niet luisteren" en "boos." Dit waren ook de meest voorkomende signalen bij incidenten die als "ernstig" gelabeld waren. 
Het identificeren van deze 16 meest voorkomende waarschuwingssignalen is een eerste stap naar een meer gestructureerde wijze van het herkennen van aankomende agressie bij kinderen, maar meer praktijkonderzoek is nodig. Er zijn wel al toepassingen denkbaar voor de praktijk. Zo kunnen de signalen worden toegevoegd als meerkeuze optie op de meldingsformulieren, helpen bij het opstellen van persoonlijke deescalatieplannen van kinderen en ondersteunen bij agressietraining van zowel studenten als professionals.

\section{De Kennedy As V: Klinimetrische eigenschappen wanneer beoordeeld door verpleegkundigen}

Hoofdstuk 3 gaat over risicotaxatie instrumenten. Deze instrumenten kwantificeren het risico op het ontstaan van agressie. Het landelijke project "Terugdringen Dwang en Drang" adviseerde om een dergelijk instrument te gebruiken in de klinische praktijk. Hierover werd een literatuurstudie gedaan. De gevonden instrumenten bleken echter minder praktisch dan een instrument wat niet vaak onderzocht is: de Kennedy As $\mathrm{V}$. De Kennedy As V is een risicotaxatie instrument maar geeft ook inzicht in het functioneren van patiënten op zeven gebieden.

Het doel van dit onderzoek is het meten van de interbeoordelaars betrouwbaarheid en klinische bruikbaarheid van de Kennedy As V, wanneer gescoord door verpleegkundigen in de dagelijkse praktijk. Dit cross-sectionele onderzoek werd gedaan op een kliniek en dagkliniek voor volwassenen en een jeugdkliniek binnen een universitair medisch centrum. De interbeoordelaars betrouwbaarheid werd gemeten door het vergelijken van scores van steeds twee verpleegkundigen, onafhankelijk van elkaar, voor één patiënt over dezelfde periode. De klinische bruikbaarheid werd gemeten met een vragenlijst.

De resultaten laten een redelijk goede interbeoordelaars betrouwbaarheid zien. Zowel de intraclass correlatie coëfficiënt (ICC) als Pearson's $r$ waren 0.79. Uit de vragenlijst over klinische bruikbaarheid bleek dat het gemiddeld 15 minuten duurde om het instrument te scoren. Dit werd aanzienlijk korter naarmate de verpleegkundige meer ervaring had in het scoren. De respondenten vonden het prettig om de lijst als houvast te gebruiken bij het observeren en rapporteren. Er waren wel problemen met het scoren van het instrument en het gebruiken van interventies gebaseerd op de scores.

Vooral de medewerkers van de adolescentenkliniek rapporteerden moeilijkheden. Na een maand werd het onderzoek daar gestopt. Om inzicht te krijgen in de problemen hielden we focusgroepinterviews, aanvullend op de oorspronkelijke onderzoeksopzet. Uit deze interviews bleek dat de medewerkers de handleiding van 
het instrument ontoereikend vonden om onderscheid te maken tussen problemen en de normale ontwikkeling van adolescenten. Ook waren er knelpunten rondom de implementatie. De medewerkers van de adolescentenkliniek benoemden wel positieve aspecten, zoals de specifieke punten om (agressief) gedrag te observeren.

We concluderen dat de Kennedy As $\mathrm{V}$ een goede interbeoordelaars betrouwbaarheid heeft wanneer afgenomen door verpleegkundigen. Dit instrument is bruikbaar om agressie vroegtijdig in te schatten en de objectiviteit van klinische beslissingen te vergroten, mits zorgvuldig geïmplementeerd.

\section{Agressie, vijandigheid en gerelateerde zorgbehoeften bij patiënten met psychotische stoornissen: een 6- jarige follow up studie}

Hoofdstuk 4 gaat over agressie en gerelateerde zorgbehoeften bij patiënten met een psychotische stoornis. Agressie is onder andere gerelateerd aan vijandigheid, suïcidaliteit en impulsiviteit. Ook zijn er associaties met andere factoren zoals trauma in de kindertijd. Hier is wel onderzoek naar gedaan, maar dat was vooral retrospectief.

Het doel van dit onderzoek is om de incidentie, persistentie, risicofactoren en het deel wat toegeschreven kan worden aan andere symptomen te onderzoeken van diverse uitkomstmaten van agressie bij patiënten met een psychotische stoornis. Met prospectieve follow-up data uit de Genetic Risk and Outcome of Psychosis (GROUP) studie deden we een survival analyse. De uitkomstmaten waren: 1) item vijandigheid uit de Positive And Negative Syndrome Scale (PANSS), 2) zorgbehoefte "veiligheid voor anderen" uit de Camberwell Assessment of Needs Short Appraisal Schedule (CANSAS) en 3) een losstaand item specifiek gericht op eventuele mishandelingen die de patiënt heeft gedaan.

De resultaten tonen dat van de 1119 patiënten, 20\% ooit iemand mishandeld had. De jaarlijkse incidentie was als volgt: vijandigheid $2.8 \%$, zorgbehoefte "veiligheid voor anderen" $0.8 \%$ en voor mishandeling was dit $1.8 \%$. De drie uitkomstmaten waren geassocieerd met elkaar, maar er waren verschillen tussen de uitkomstmaten. Factoren geassocieerd met de drie uitkomstmaten waren: impulsiviteit, gebrek aan samenwerking, het aantal onvervulde zorgbehoeften op de CANSAS, suïcidale gedachten en het mannelijk geslacht.Vijandigheid en mishandeling waren geassocieerd met trauma's in de kindertijd; zorgbehoefte "veiligheid voor anderen" was dat niet.

Concluderend zijn agressie en aanverwante zorgbehoeften met elkaar geassocieerd. Enkele reeds bekende risicofactoren zijn bevestigd, waaronder trauma in de kindertijd. Dit wijst erop dat agressie een complex fenomeen is en verschillende ontstaanswijzen kent. 


\section{Vijandigheid en agressie in eerste episode psychose: resultaten uit de OPTiMisE trial}

In hoofdstuk 5 richten we ons op patiënten met een eerste psychotische episode. Patiënten hebben tijdens een eerste psychose een verhoogd risico op agressie. We kijken naar zowel de klinische factoren die geassocieerd zijn aan agressie, als naar de effectiviteit van antipsychotica op agressie.

Het doel van dit onderzoek is om de klinische factoren te identificeren die geassocieerd zijn met agressie en om de effecten van antipsychotica op agressie te onderzoeken bij patiënten met een eerste psychose. Hiervoor gebruikten we data uit de Optimization of Treatment and Management of Schizophrenia in Europe (OPTiMiSE) studie. In fase I van deze studie kregen alle patiënten vier weken openlabel amisulpride. Wanneer patiënten hierna niet in remissie waren, gingen zij door naar de dubbelblinde fase II, waar zij werden gerandomiseerd op continueren van amisulpride of een switch naar olanzapine. In deze analyse gebruikten we twee uitkomstmaten voor agressie: 1) vijandigheid item van de PANSS en 2) domein storend en agressief gedrag van de Personal and Social Performance scale (PSP).

De baseline meting bij 446 patiënten liet zien dat agressie significant geassocieerd was met PANSS items wanen, hallucinaties, opwinding, spanning, gebrek aan samenwerking, ongewone gedachteninhoud, gebrek aan ziekte-inzicht en impulsiviteit. Voor de analyse over antipsychotica gebruikten we een subgroep van patiënten, die bij de baseline een PANSS score vijandigheid $>1$ hadden. Deze groep bestond uit 185 (41.5\%) patiënten. Tijdens fase I verbeterden de scores aanzienlijk. Dit effect bleef significant wanneer gecorrigeerd voor positieve symptomen. Er kon echter geen significant verschil gevonden worden tussen olanzapine en amisulpride in fase II van de studie.

We concluderen dat de prevalentie van agressie in deze steekproef laag is. De relatie tussen agressie en andere klinische symptomen is bevestigd. Tot slot bleek amisulpride effectief voor de behandeling van agressie bij patiënten met een eerste psychose.

\section{Effectiviteit van typische versus atypische antipsychotica op vijandigheid bij patiënten met psychotische stoornissen: een review en meta-analyse}

In het zesde hoofdstuk onderzochten we de effectiviteit van antipsychotica op vijandigheid bij patiënten met een psychotische stoornis. Hoewel clozapine het meest effectief lijkt tegen agressie, is het bewijs hiervoor onvoldoende gedocumenteerd. 
Het doel van deze review en meta-analyse is het bepalen van het effect van typische versus atypische antipsychotica op agressie.

De initiële zoekstrategie resulteerde in 1395 studies. Na selectie bleven 18 gerandomiseerde studies over. Deze vergeleken minimaal één typisch met minimaal één atypisch antipsychoticum gedurende 4 weken of langer bij patiënten met een psychotische stoornis. De meta-analyse van deze 18 studies liet een klein maar significant voordeel zien voor atypische in vergelijking met typische antipsychotica. De heterogeniteit tussen de studies was echter hoog. De heterogeniteit was veel lager bij een analyse van alleen de niet door de industrie gesponsorde studies. Een differentiatie tussen studies met hoog en laag gedoseerde antipsychotica liet zien dat in de groep met hoog gedoseerde antipsychotica een significant verschil was tussen de typische en atypische antipsychotica maar niet in de laag gedoseerde groep. Clozapine had de hoogste effect size en een lage heterogeniteit.

We concluderen dat atypische antipsychotica, en vooral clozapine, effectiever zijn tegen agressie bij patiënten met een psychotische stoornis in vergelijking met typische antipsychotica. Hiermee bevestigen we eerdere bevindingen. Deze resultaten zijn belangrijk voor het maken van klinische beslissingen in de dagelijkse praktijk.

\section{Conclusie}

Agressie in de psychiatrie is een complex begrip. Een dergelijk ingewikkeld probleem kent niet één gouden oplossing. De afgelopen jaren is er steeds meer aandacht en onderzoek geweest naar dit onderwerp. Daarnaast zijn er belangrijke nieuwe ontwikkelingen gaande, zoals het terugdringen van eenzame opsluiting en de opkomst van de Intensieve Zorg voor de psychiatrie.

Dit proefschrift levert enkele klinisch relevante bevindingen. Deze bevindingen kunnen bijdragen aan het begrijpen, signaleren, inschatten en behandelen van agressie, maar meer onderzoek is nodig. Hoewel agressie mogelijk altijd een probleem zal blijven binnen de psychiatrie, hopen we dat een continue focus en implementatie van nieuwe ontwikkelingen en onderzoeksresultaten, zal bijdragen aan een verdere reductie van incidenten of in ieder geval aan de ernst van de incidenten. 

Dankwoord 



\section{Dankwoord}

Graag wil ik als eerst de patiënten bedanken die hebben deelgenomen aan de verschillende onderzoeken. Onderzoeken zoals deze zijn onmogelijk zonder jullie inzet. Ik heb veel bewondering voor de tijd en energie die jullie hiervoor vrij hebben gemaakt.

Jaren geleden begon dit traject met een onderzoeksvraag vanuit de afdeling. Tegelijkertijd had ik de wens om mijzelf verder te ontwikkelen in wetenschappelijk onderzoek. Dit proefschrift is zich toen langzaam maar zeker gaan ontvouwen. Soms was dat best even zoeken. Gelukkig heb ik veel hulp en ondersteuning gehad. Het is voor mij erg waardevol geweest om met zoveel verschillende mensen samen te mogen werken. Ieder met zijn eigen visie, kennis en ervaring, waar ik veel van heb mogen leren. Ik wil dan ook iedereen bedanken die mij heeft geholpen in dit traject: bij het uitvoeren van de onderzoeken, het analyseren van de data en het schrijven van de papers.

Graag wil ik mijn promotoren, Jim van Os en Floortje Scheepers, bedanken. Beste Jim, dank dat je mij meenam op het laatste stuk van dit traject. Je hebt mij enorm geholpen een geheel te maken van de verschillende onderdelen. Dat gaf mij inspiratie en nieuwe inzichten. Floortje, dank dat ik op je kon rekenen en dat je er was wanneer nodig. Je gaf mij de ruimte en het vertrouwen om dit proefschrift af te ronden, dat is erg belangrijk voor mij geweest.

Een belangrijk deel van dit proefschrift schreef ik met Iris Sommer. Beste Iris, zonder jou was dit nooit tot stand gekomen. Je gaf mij het vertrouwen om dit proefschrift te gaan schrijven. Dank voor je steun, je goede adviezen en al je kennis en ervaring waarmee je mij hebt geïnspireerd.

Dan wil ik Thóra Hafsteinsdóttir bedanken. Beste Thóra, dank voor je steun en alles wat ik van je heb mogen leren. Je kritische blik en enthousiasme hebben mij enorm geholpen bij het schrijven van mijn eerste artikel. Floor Gooskens en Roland van de Sande, dank voor de fijne samenwerking en jullie inspanningen tijdens ons project.

Marije Valenkamp en Henk Nijman: bedankt voor de fijne samenwerking tijdens het analyseren van de gegevens en het schrijven van ons paper. Het was een boeiend proces en ik heb veel van jullie geleerd. 
Pál Czobor, thank you for your help in conducting and writing our meta-analyses. I learned a lot from your experience and expertise.

The co-authors of the OPTiMiSe paper: Celso Arango, Covadonga Díaz-Caneja, Gregor Berger, Stefan Leucht, Julio Bobes, Pilar Sáiz and María Paz García-Portilla, thank you for your critical comments and helpful review on our paper. Caroline van Baal, dank voor je hulp bij de analyse van de data en dat je de tijd nam om steeds uitleg te geven over de procedures, dat was erg leerzaam en interessant.

De GROUP auteurs, Therese van Amelsvoort, Agna Bartels-Velthuis, Richard Bruggeman, Wiepke Cahn, Lieuwe de Haan, Frederike Schirmbeck, Claudia Simons en Jim van Os wil ik danken voor de jarenlange inspanningen voor deze mooie dataset en voor het meedenken en de waardevolle reacties op onze analyse en het paper. Zimbo en Joyce, dank voor jullie geduld, tijd en aandacht om mij te helpen met allerhande (data) vragen.

De beoordelingscommissie, prof.dr. J.M.A.Visser-Meilij, prof.dr.M.J.Schuurmans, prof. dr. L. Schoonhoven, prof. dr. B. van Meijel en dr. M.C. Marcelis, wil ik graag bedanken voor het lezen en beoordelen van het manuscript.

De collega's met wie ik tijdens diverse trials heb gewerkt wil ik bedanken. Inge, voor al je hulp en ondersteuning tijdens de trials, voor het meedenken over ons OPTiMiSE paper en je kritische blik daarop. Paula, voor het delen van je kennis en ervaring rondom onderzoek en natuurlijk voor alle gezelligheid. Resy, Jocelyn, Charissa, Lyliana, Cynthia \& Margot: dank voor de fijne samenwerking.

Ans, Annick en Janneke, jullie waren onmisbaar bij het plannen en regelen van allerlei zaken rondom de afronding van dit proefschrift, veel dank daarvoor.

Vanuit het PhD-programma Clinical \& Experimental Neuroscience, wil ik graag Geert en Krista bedanken voor alle hulp en ondersteuning tijdens dit traject.

Veel collega's van de afdeling Psychiatrie van het UMC Utrecht hebben mij gesteund of geholpen gedurende dit traject. Lena, je gaf mij de ruimte om dit te voltooien. Dank voor al je steun en advies, al zoveel jaren. Mijn kamergenoten en collegabeleidsmedewerkers, Irina, Koen, Desiree en Marleen: dank voor alle gezelligheid op onze kamer, de humor en behulpzaamheid. Irina, voor je goede adviezen, het meelezen en het delen van je ervaringen rondom jouw promotietraject, daar heb ik veel aan gehad. Sabine Stigter, voor de ruimte en de flexibiliteit die je mij gaf om 
het klinisch werken goed te combineren met het doen van onderzoek. Ine, voor je gezelligheid, geduld rondom mijn altijd wijzigende roosters en je bereidheid om mee te denken in oplossingen. Liesbeth, al vanaf de tijd dat we nog samen op A3 werkten heb ik veel van je geleerd en heb je mij geïnspireerd met je kennis en visie. Jij hebt mij altijd gestimuleerd om hiermee door te gaan. Veel dank daarvoor. Het PsyData team wil ik bedanken, met in het bijzonder Karin en Femke. Ik ben heel blij met jullie expertise binnen de afdeling. Dank voor de fijne samenwerking de afgelopen jaren.

De collega's van zorglijn A3 hebben mij enorm geholpen bij het uitvoeren van het onderzoek naar de Kennedy. Zoals geen andere afdeling dat kan, hebben jullie je hiervoor ingezet. Dank Rina, Josette, Laura, Peter, Chris, Rosanne, Ingrid en de rest.

De collega's van zorglijn A2 wil ik graag bedanken. Alan, Aukje en Roland, jullie geven richting aan het team in soms roerige tijden. Dank voor de fijne samenwerking. Alle verpleegkundigen van A2, voor de gezellige, hectische en soms vreemde diensten op de afdeling en de gezelligheid daarbuiten: Stephan, Renske, Desiree, René, Heimen, Marne, Frank, Franka, Nienke, Daniël, Miranda en alle nieuwe(re) collega's. Speciaal natuurlijk dank aan Berivan en Wouter, die inmiddels veel meer zijn dan alleen collega's: voor jullie vriendschap, humor en eerlijkheid. Jullie zorgden voor de nodige ontspanning en luchtigheid tijdens het schrijven. Patricia en Nienke, jammer dat jullie de Psychiatrie hebben verlaten, maar ik ben blij dat we nog contact hebben. Dank voor alle gezelligheid.

Dan zijn er nog veel meer collega's die zorgen voor gezelligheid, inspirerende gesprekken in de wandelgangen en een aangename werksfeer: Renate, Carry, Bart, Margit, Lucy, Aumer, Yvonne, Fleur, Annemarie, Ilona en alle andere collega's van de secretariaten, de artsen en de psychiaters. Natuurlijk ook dank aan alle verpleegkundigen van onze afdeling, die elke dag klaarstaan om voor onze patiënten te zorgen.

Tijdens dit hele traject, maar zeker in het laatste deel, is gezelligheid en ontspanning essentieel geweest. Ik wil dan ook graag mijn vrienden bedanken. Tine, voor je vriendschap, al sinds de $\mathrm{HBO}-\mathrm{V}$ en ondanks de compleet verschillende afdelingen waar we werken. Bianca, voor je eerlijkheid, gezelligheid en vriendschap al die jaren. Susanne, voor al je gezelligheid, eigenzinnigheid en steun. Marije, voor alle leuke dingen die we hebben gedaan, alleen helaas nu op afstand. We komen snel weer een keer langs om in de bergen te wandelen!

Tim, Tim, Tim, Eva, Anke, Bart, Joyce, Marius, Swen en Janneke: voor jullie jarenlange vriendschap, alle gezelligheid, de leuke avonden, weekenden en vakanties. Speciaal 
natuurlijk dank aan mijn paranimfen. Ik ben ontzettend blij dat jullie mij hebben geholpen in het laatste stuk van dit traject. Tim, met je humor, je stimulans en je hulp werd de afronding toch nog een feest. Janneke, dank voor je al hulp, tijdens het hele traject, en natuurlijk voor het meedenken over de omslag samen met Swen. Door al die jaren heen heb je mij gestimuleerd om mijn hart te volgen en hiermee door te gaan. Ik ben heel dankbaar voor onze vriendschap.

Mijn schoonfamilie, Kees, Susanne, Menno, Elisa en Roel: met jullie heb ik zoveel gezelligheid erbij gekregen. Dank voor jullie betrokkenheid de afgelopen jaren.

Van mijn familie heb ik veel steun ervaren tijdens dit traject. Graag wil ik dan ook iedereen bedanken die in de loop der jaren zoveel interesse heeft getoond. In het bijzonder mijn lieve oma's. Zij zijn, op een heel verschillende manier, voor mij een groot voorbeeld geweest. Ik ben dankbaar dat zij een belangrijk deel van mijn leven waren.

Roos, ik ben dankbaar dat jij mijn zus bent. Ik heb bewondering voor wie je bent en ben trots op hoe je in het leven staat. Cyriel, jij maakt het allemaal nog leuker. Dank dat jullie er voor mij zijn, voor al jullie humor, steun en gezelligheid.

Pa en ma, van jullie leerde ik mijn eigen weg te volgen. Zo is dit proefschrift tot stand gekomen. Mijn dank aan jullie past niet in een paar zinnen. Jullie zijn er, en ik ben dankbaar dat jullie er altijd zijn, met liefde, steun, raad en daad.

Kristiaan, jij maakt het compleet. Dank voor je positieve kijk op het leven, voor al onze avonturen en je onvoorwaardelijke steun. Natuurlijk ook voor je hulp bij het maken van dit boek. $\mathrm{Nu}$ is het tijd voor iets nieuws! 



\section{Curriculum Vitae}





\section{Curriculum Vitae}

Margo Faay was born on September 12 $2^{\text {th }}$, 1987, in Waddinxveen, the Netherlands. She started her nursing education in 2004 at the University of Applied Sciences Leiden while working as a nursing student at the Robert Fleury Stichting (later: GGZ Haagstreek, Rivierduinen) in Leidschendam. In 2008, she received her bachelor's degree in nursing and started working as a registered nurse at the Department of Psychiatry, University Medical Center (UMC) Utrecht. In 2011, she obtained her Masters' degree in Nursing Sciences under the supervision of dr. T.B. Hafsteinsdóttir. She worked as a project assistant and later as the project manager for a seclusion reduction project within the UMC Utrecht. After this project was successfully completed, she became involved in a number of clinical trials where she worked as a research assistant under the supervision of prof. dr. I.E. Sommer. From 2017-2018, she investigated the implementation of the CrisisKaart in Utrecht for the Steunpunt GGZ Utrecht under supervision of prof. dr. F.E. Scheepers. In 2018, her PhD trajectory was formalized when Margo had the opportunity to cluster her previous research activities. She undertook the necessary training and supplementary research activities. Her PhD trajectory was finished under the supervision of prof. dr. F.E. Scheepers and prof. dr. J.J. van Os. Currently, Margo is a policy advisor within the Department of Psychiatry of the UMC Utrecht. Many of her current projects focus on aggression and the implementation of the new High- and Intensive care unit (HIC). From 2008 on, she works part-time as a nurse at the department of Psychiatry, UMC Utrecht, as she considers it essential to keep in touch with daily practice. 


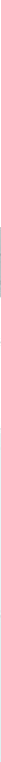

称 UMC Utrecht 